Surveys in Differential Geometry XIX

\title{
Regularity of stable minimal hypersurfaces: recent advances in the theory and applications
}

\author{
Neshan Wickramasekera \\ This article is dedicated to Richard Hamilton, Leon Simon and Karen Uhlenbeck \\ in recognition of their fundamental work in Geometric Analysis.
}

\begin{abstract}
We survey some recent progress in the regularity theory of stable minimal hypersurfaces, describing also earlier work related to the recent results, some applications of the recent results, and key aspects of their proofs.
\end{abstract}

\section{Contents}

\section{INTRODUCTION}

Main recent results and their historical context 233

Optimality 235

Applications $\quad 236$

Geometric aspects of the proof 238

$\begin{array}{ll}\text { Structure of the rest of the paper } & 241\end{array}$

Part 1. Stationary VARIFOlds, Their Singularities, AND THE CODIMENSION 1 THEORY FOR STABLE VARIFOLDS

1. The Allard-Almgren theory of varifolds 242

1.1. Smooth minimal submanifolds 242

$\begin{array}{ll}\text { 1.2. Varifolds } & 243\end{array}$

1.3. First variation of a varifold and stationarity 245

1.4. The monotonicity formula 245

1.5. The Allard-Almgren Compactness Theorem 246

2010 Mathematics Subject Classification. 35-XX, 53-XX.

Key words and phrases. varifolds, currents, stable minimal hypersurfaces, first variation, second variation, stationarity, stability, tangent cones, regularity theory, classical singularities, branch points, maximum principle, unique continuation, phase transition, blow up. 
1.6. Upper semi-continuity of density 246

1.7. Tangent cones and the question of their uniqueness 247

1.8. Interior regularity of stationary varifolds: Allard's theorems 248

2. Singularities of stationary varifolds 250

2.1. The Almgren-Federer Generalized Stratification 251

2.2. Classical singularities and branch points 252

2.3. Multiplicity 1 classes: Simon's work 253

3. Stable varifolds 256

3.1. Second variation and stability 256

3.2. Stability inequality in codimension 1

3.3. Non-existence of singular stable hypercones in low dimensions:

Simons' theorem 257

3.4. Area minimizing hypersurfaces: the work of De Giorgi, Federer-Fleming, Federer and Hardt-Simon 258

3.5. The Bernstein Theorem 258

3.6. Curvature estimates under a priori regularity hypotheses: the work of Schoen, Schoen-Simon-Yau and Schoen-Simon 259

3.7. A general theory: stable codimension 1 varifolds with no classical singularities 261

4. Some applications of the general theory 262

4.1. Strong maximum principle for stationary codimension 1 varifolds 262

4.2. Unique continuation for stationary codimension 1 varifolds 264

4.3. Remarks on the proofs of the maximum principle and the unique continuation theorem 264

4.4. Interface regularity for a class of phase transition problems 266

5. Some questions arising from the recent work 267

Part 2. An outline of the proof of Theorem 3.3 (REGULARITY AND COMPACTNESS FOR STABLE CODIMENSION 1 VARIFOLDS WITH NO CLASSICAL SINGULARITIES)

6. Basic strategy and main steps

6.1. The Sheeting Theorem and the Minimum Distance Theorem 269

6.2. Proof of Theorem 3.3 using the Sheeting Theorem and the Minimum Distance Theorem

6.3. Proof strategy for the Sheeting Theorem and the Minimum Distance Theorem: Induction 272

6.4. An elementary consequence of the induction hypotheses 274

7. Step (i): Induction for the Sheeting Theorem 275

7.1. Linear theory: proper blow-up classes 275

7.2. Coarse blow-ups of stationary varifolds 277

7.3. Elementary properties of coarse blow-ups 279

7.4. Minimum Distance Property for coarse blow-ups: Part I 280

7.5. Minimum Distance Property for coarse blow-ups: Part II 282 
7.6. Minimum Distance Property for coarse blow-ups: Part III

7.7. Sketch of the proof of Theorem 6.1 subject to the induction hypotheses

8. Steps (ii) and (iii): Induction for the Minimum Distance Theorem (and the overall completion of induction) 296

References

\section{INTRODUCTION}

Regularity and singularity theory of minimal submanifolds of Riemannian manifolds is a subject with a rich history and a large number of interesting, central open questions. Apart from producing beautiful, deep results with many important direct applications in geometry and analysis, this subject has been the birthplace of an array of fundamental techniques of far reaching significance. These widely applicable techniques have served as a catalyst for a substantial volume of research in a diverse range of areas in Geometric Analysis and non-linear PDE, such as the variational theories of harmonic maps, Yang-Mills connections, free boundary and phase transition problems; their associated gradient flows; other geometric flows including Ricci flow, mean curvature flow and Yamabe flow, and general relativity, to name just a few.

In this brief survey our principal aim is to discuss some recent progress in the theory of stable minimal hypersurfaces, focusing in particular on the work [Wic14a]. We shall also briefly survey several other results in minimal surface theory along the way, choosing among the many important theorems only the ones that are directly related - implicatively or contextually — to the work [Wic14a].

Main recent results and their historical context. The pioneering work in the study of stable minimal hypersurfaces was done by De Giorgi, whose work [DG61], published in 1961, established that an $n$-dimensional locally area minimizing boundary in a Riemannian manifold is smoothly embedded away from a closed (interior) singular set $\Sigma$ of $n$-dimensional Hausdorff measure zero. This groundbreaking regularity result triggered a large amount of activity in the theory of minimal submanifolds and related areas. In particular, during the decade 1960-70, the regularity conclusion of De Giorgi's theorem itself went through a step-by-step process of being strengthened, as a result of the work of Federer-Fleming ([FF60]), Reifenberg ([Rei60 $])$, Fleming ([Fle62 $])$, Almgren ([Alm66]), Simons ([SJ68 $]$ ) and Federer ([Fed70]), and was shown to be valid for area minimizing codimension 1 rectifiable currents (away from their boundary), for which an existence theory (in homology classes or subject to fixed boundary) had been developed in the seminal work of Federer-Fleming ([FF60]). The end result of all of this was one of the most beautiful theorems in Geometric Analysis: the 
(interior) singular set $\Sigma$ of the minimizer is in fact empty if $n \leq 6$, discrete if $n=7$ and has Hausdorff dimension $\leq n-7$ if $n \geq 8$. Soon after this fundamental result had been established, Bombieri-De Giorgi-Giusti ([BDG69]) showed that the cone over the product of spheres $\frac{1}{\sqrt{2}} \mathbb{S}^{3} \times \frac{1}{\sqrt{2}} \mathbb{S}^{3} \subset \mathbb{R}^{8}$, which Simons' had already observed to be stable, is in fact locally area minimizing. Thus interior singularities do occur in locally area minimizing 7-dimensional hypersurfaces, establishing the fact that the above Hausdorff dimension bound on the singular set is in fact the best possible general conclusion.

The work [Wic14a] sharpens this classical theory, by significantly weakening its hypotheses. This work shows that one needs to verify surprisingly little to draw the same regularity conclusion, and that the result in fact holds in the more general varifold setting. Specifically, the main theorem of [Wic14a] shows that exactly the same conclusion on the size of the singular set continues to hold under the following three conditions which together form a sharp hypothesis that is considerably weaker than the area minimizing assumption:

(i) The hypersurface (by which we mean a codimension 1 integral varifold; see the definition in Section 1 below) is stationary in the sense that it has zero first variation with respect to the area functional for any smooth ambient deformation that leaves the region outside some compact set fixed.

(ii) The regular part (the smoothly embedded part of the support) of the hypersurface is stable in the sense that the hypersurface has non-negative second variation with respect to the area functional for any smooth ambient deformation as in (i) that also leaves a neighborhood of the singular set (the complement of the regular part in the support of the hypersurface) fixed and moves the regular part in the normal direction. (Note that the regular part is always non-empty by Allard's regularity theorem, but just under the stationarity hypothesis it is not known if the singular set has $n$-dimensional Hausdorff measure zero, where $n$ is the dimension of the hypersurface.)

(iii) A certain type of singularities, which we shall call classical singularities, are entirely absent from the hypersurface. A classical singularity of an $n$-dimensional surface is a singular point $Z$ of the surface about which there is a neighborhood in which the surface is the union of embedded sheets coming together smoothly along a common $(n-1)$-dimensional free boundary containing $Z$.

See Sections 1.1 and 3.1 for the precise definitions of stationarity and stability respectively, and Section 2.2 for the precise definition of classical singularity. 
The work [Wic14a] also establishes a compactness theorem: any uniformly mass-bounded subset of the collection of stationary, stable hypersurfaces with no classical singularities is compact with respect to measuretheoretic (i.e. varifold) convergence.

These regularity and compactness results and the precise hypotheses needed for them are stated as Theorem 3.3 below. Note in particular (see the statement of Theorem 3.3) that the actual stability hypothesis needed for these results is a priori even weaker than what is stated in (ii) above. This is an important point because the weaker stability hypothesis makes it possible to use in the proofs the stability inequality (i.e. the inequality in the section entitled "Geometric aspects of the proof" below) without having to make any orientability hypotheses on the regular part of the hypersurface.

For the purposes of the rest of this introduction, we now state a version of Theorem 3.3 in mildly imprecise language, in which the stability hypothesis is not explicitly stated in the weaker form referred to above:

Theorem 1. ([Wic14a]) The support of a stationary, stable $n$-dimensional integral varifold $V$ on an $(n+1)$-dimensional smooth Riemannian manifold $U$ is a smoothly embedded hypersurface away from a closed set $\operatorname{sing} V$ (the singular set of $V$ ) of Hausdorff dimension $\leq n-7$ (which is empty if $n \leq 6$ and discrete if $n=6$ ) provided $V$ has no classical singularities. Moreover, the family of stationary, stable $n$-dimensional integral varifolds on $U$ with no classical singularities and locally uniformly bounded mass is compact in the topology of varifold convergence.

An important special case of this result was established in 1981 by Schoen and Simon in [SS81], where in place of the no-classical-singularities hypothesis a more restrictive condition was assumed, namely, that the singular set (of the stable hypersurface) has locally finite $(n-2)$-dimensional Hausdorff measure.

It is easy to see a priori, by a straightforward comparison surface construction, that a codimension 1 area minimizing rectifiable current with no boundary cannot have a classical singularity, so Theorem 1 does indeed subsume the aforementioned regularity theory for area minimizers.

Optimality. The above theorem is sharp with respect to all of its hypotheses and, as already pointed out, also with respect to its conclusion (in view of the cone over $\frac{1}{\sqrt{2}} \mathbb{S}^{3} \times \frac{1}{\sqrt{2}} \mathbb{S}^{3}$ in $\mathbb{R}^{8}$ ). Its sharpness with respect to the no-classical-singularities hypothesis is evident in view of simple examples such as transversely intersecting pairs of hyperplanes in a Euclidean space. The cone over the Clifford torus $\frac{1}{\sqrt{2}} \mathbb{S}^{1} \times \frac{1}{\sqrt{2}} \mathbb{S}^{1}$ in $\mathbb{R}^{4}$ shows that the stability hypothesis of the theorem is necessary for the codimension 7 conclusion on the singular set.

Indeed in the absence of stability, for stationary $n$-dimensional hypersurfaces even almost everywhere regularity remains a major open problem even in the case $n=2$ and subject to the no-classical-singularities hypothesis. 
Thus the stability hypothesis of the theorem is a restriction only on a potentially very small part of the (stationary) hypersurface, namely the regular part, in contrast to the case where an appropriate a priori size restriction on the singular set is imposed, e.g. as in [SS81]. This is why it is surprising that stability on the regular part in conjunction with the no-classical-singularities hypothesis is all that is needed for the sharp conclusions of the theorem. (See the section entitled "Geometric aspects of the proof" below for a more detailed discussion of this point.)

In higher codimension, the best possible regularity conclusion one could hope for for $n$-dimensional stable varifolds with no classical singularities is that their singular sets have Hausdorff dimension $\leq n-2$. This is known to be true for area minimizing rectifiable currents with no boundary by the grand work of Almgren [Alm83], in which case the no-classical-singularities hypothesis is automatically satisfied. Furthermore, the result in this case is already sharp in view of the many two dimensional examples of oriented area minimizers provided by singular complex analytic varieties (such as $\{(z, w): z w=0\} \cap \mathbb{C} \times \mathbb{C}$ or $\left.\left\{(z, w): z^{2}=w^{3}\right\} \cap \mathbb{C} \times \mathbb{C}\right)$ which are all calibrated and consequently locally area minimizing. (See also the recent papers of De Lellis and Spadaro [DS, DS11] which present Almgren's lengthy proof concisely in a more accessible, step-by-step form, making also interesting connections between some of Almgren's key estimates and ideas in metric geometry and PDE).

Applications. The fact that Theorem 1 requires no a priori size hypothesis on the singular set and that the stability hypothesis is required to hold only on the regular part are very useful features for its applications. In particular, one needs no a priori information on the possible degenerate singular points (i.e. branch points) where there is a planar tangent cone; one only needs to verify the no-classical-singularities hypothesis which in principle is easier to check, and the theorem then guarantees, among other things, non-existence of such degenerate singularities.

Note also that the no-classical-singularities hypothesis is a condition requiring ruling out a certain type of local structure of the support of the varifold, as opposed to being an "asymptotic hypothesis" requiring ruling out various types of tangent cones. Conventionally, the latter has been the case in many results in the literature related to controlling the size of a singular set. This difference is significant if for no other reason than the fact that it means that in applying the theorem to an $n$-dimensional, codimension 1 varifold with stable regular part, it suffices to verify the no-classical-singularities hypothesis (and in particular to rule out tangent cones supported on unions of three or more half-hyperplanes meeting along a common axis) away from any subset $Z$ of the support of the varifold with $Z$ having $(n-1)$-dimensional Hausdorff measure zero, regardless of whether $Z$ is known to be closed; that is to say, it suffices to verify that no point in the complement of such a subset $Z$ of the support is a classical singularity. This observation has indeed been 
crucial in the recent application of the theorem to phase transition problems (see below and also Section 4.4).

Theorem 1 has already found applications in a variety of interesting situations. A long conjectured strong maximum principle and a unique continuation theorem for stationary codimension 1 integral varifolds follow from the theorem $([\mathbf{W i c 1 4 b}])$. These two results are sharp with respect to the size of the allowable singular sets, and they require Theorem 1 not in its full strength but in the special case when the $(n-1)$-dimensional Hausdorff measure of the singular set is zero (which very directly implies the noclassical-singularities hypothesis but does not seem to lead to a significantly easier proof; see the discussion in the section entitled "Geometric aspects of the proof" below). Both the strong maximum principle and the unique continuation theorem of [Wic14b] build upon the important earlier work of Moschen ([Mos77]), Simon ([Sim87]), Solomon-White ([SimWic]) and Ilmanen ([Ilm96]) that had investigated special cases of the maximum principle.

Theorem 1 in its full strength has been crucial for settling the interface regularity question associated with a class of phase transition problems. Specifically, in the work of Tonegawa and the author ([TW10]), regularity of the limit-interfaces associated with stable critical points of the Cahn-Hilliard (or Allen-Cahn) functionals has been shown to follow from, and require, the full force Theorem 1 . This result extends earlier work on the $\Gamma$-convergence properties of the functionals (and hence restricted to the context of energy minimizers) due to many authors including Modica, Mortola, Sternberg and Kohn ([Mod87], [MM77], [Ste88], [KS89]). It builds upon the work of Hutchinson-Tonegawa ([HT00]) and Tonegawa ([Ton05]) that brought in, influenced by the work of Ilmanen $([\mathbf{I l m 9 3}])$ in the parabolic setting, a new perspective to the elliptic problem based on the theory of varifolds, opening up the study of the behavior of non-minimizing critical points.

An obvious question that arises in light of the work [TW10] is whether the well-known Almgren-Pitts-Schoen-Simon existence theory ([Alm65], [Pit77], [SS81]) for embedded minimal hypersurfaces in compact Riemannian manifolds can be recast, and perhaps also made simpler (given, of course, Theorem 1) and strengthened, with an approach based on the Cahn-Hilliard functional. (See Section 5 below).

We mention finally that in the striking recent work of Colding-Minicozzi, Theorem 1 has been shown to play a role in the study of singularities of mean curvature flow of initially embedded hypersurface. Specifically, it is used in [CM14] to show that for $2 \leq n \leq 6$, an $n$-dimensional self-shrinker in $\mathbb{R}^{n+1}$ with polynomial volume growth and no classical singularities, whose regular part satisfies a certain stability property (called $F$-stability, see [CM14]) with respect to Gaussian area, must be supported on either a hyperplane or a round sphere. This classification result is expected to play an important role in the study of generic singularities of mean curvature flow of embedded hypersurfaces. 
See the discussion in Sections 4.1-4.4 below for more details of some of these applications, and Section 5 for some open questions arising from the work described above.

Geometric aspects of the proof. To discuss some key aspects of the proof of the above theorem, let $V$ be an $n$-dimensional stable stationary hypersurface admitting no classical singularities. The principal geometric difficulty one faces in proving the theorem arises from the possible presence of higher multiplicity in $V$. Note that the hypotheses of the theorem allow the possibility that the multiplicity function of $V$ varies on the regular part of $V$ even if the support of $V$ is connected, as well as of course on the singular set which a priori could have positive, or even proportionately large, $n$ dimensional Hausdorff measure. In particular, a priori, there could be a set of singularities of positive $n$-dimensional Hausdorff measure at each of which there is a tangent hyperplane with multiplicity $\geq 2$. (Allard's regularity theorem (Theorem 1.2 below) - a far reaching generalization of De Giorgi's work applicable to stationary varifolds - implies that any point where there is a multiplicity 1 tangent hyperplane is a regular point.) Yet as the very first step towards the conclusions of the theorem, one has to rule out such singularities completely, i.e. prove the following:

(a) no tangent cone to $V$ at a singular point can be a higher multiplicity hyperplane.

This step goes a long way towards resolving the higher multiplicity issue, and would imply that the singular set has $n$-dimensional measure zero. To get closer to the full conclusion of the theorem, one needs next the following additional result:

(b) no tangent cone to $V$ can be supported on a union of three or more $n$-dimensional half-hyperplanes meeting along a common $(n-1)$ dimensional subspace.

For oriented locally area minimizing hypersurfaces (i.e. locally area minimizing codimension 1 rectifiable currents with no boundary), (a) and (b) are relatively easy to prove and can be established independently of each other by straightforward construction of comparison surfaces. For such minimizers, De Giorgi's theorem on the other hand says that any point where there is a multiplicity 1 tangent hyperplane is a regular point, and therefore it follows from (a) that any point of an oriented locally area minimizing hypersurface where there is a tangent cone supported on a hyperplane is a regular point. With the help of (b), the compactness theorem for area minimizers and little additional thought, one can take this further to deduce that the dimension of the subspace along which a singular tangent cone to an oriented locally area minimizing hypersurface is translation invariant is at most $n-3$. This fact and Federer's dimension reducing principle quickly take us to the (intermediate) conclusion that the singular set of an oriented 
$n$-dimensional locally area minimizing hypersurface must have Hausdorff dimension $\leq n-3$.

For the larger class $\mathcal{S}$ of stable hypersurfaces $V$ with no classical singularities (as considered in the above theorem), the facts (a) and (b) as well as the relevant compactness theorem are considerably more difficult to establish and require a fundamentally different approach. In particular, the key in that case turns out to be to aim to prove (a) and (b) simultaneously by an inductive argument. In fact, the key is to prove simultaneously two results for $\mathcal{S}$ - the Sheeting Theorem (Theorem 6.1 below) and the Minimum Distance Theorem (Theorem 6.3) - that very directly imply (a), (b) as well as the compactness theorem. With that approach it is possible to establish, as shown in [Wic14a], various key a priori estimates for $V \in \mathcal{S}$ that appear to be inaccessible via an attempt, inductive or otherwise, to reach the conclusions (a) and (b) one after the other.

Once (a), (b) and the compactness theorem are in place, the full regularity conclusion can be deduced from Simons' theorem (Theorem 3.1 below, which asserts non-existence, when $2 \leq n \leq 6$, of an $n$-dimensional stable minimal hypercone with an isolated singularity) and, in case of area minimizers, Federer's dimension reducing principle, or, in case of the class $\mathcal{S}$, the elementary yet more general principle known as the Almgren-Federer Generalized Stratification Theorem (Theorem 2.1 below).

In establishing the claims (a), (b) above for $V \in \mathcal{S}$ and the compactness theorem for $\mathcal{S}$, one faces a serious geometric difficulty that stems from the extremely weak nature of the stability hypothesis. To discuss this in the right context, note first that by the definition of classical singularity, if an $n$-dimensional varifold has a classical singularity then it must have classical singularities along an $(n-1)$-dimensional submanifold. Thus for instance if the singular set of an $n$-dimensional varifold $V$ satisfies $\mathcal{H}^{n-1}(\sin g V)=0$, then $V$ cannot have classical singularities and hence, if $V$ is also stable and has codimension 1, then the above theorem would imply that its singular set has codimension $\geq 7$. Subject to the even more restrictive hypothesis that the singular set has locally finite $(n-2)$-dimensional Hausdorff measure, the conclusions of the theorem had been established, as mentioned before, by Schoen and Simon in 1981 ([SS81]).

The key a priori consequence of this simplifying assumption on the singular set, upon which the proof in [SS81] relied heavily, is that the singular set is "removable for the stability inequality for $V$ " (see below), giving strong a priori $L^{2}$ control of the second fundamental form of the hypersurface. Not having the benefit of such a priori curvature control is the most significant geometric difficulty one faces in proving (a), (b) for $V \in \mathcal{S}$ and the compactness theorem for $\mathcal{S}$.

To elaborate on this point further, let $V$ be as in the theorem above and let $M$ denote its regular part. Note that since $V$ is stationary, we have by Allard's regularity theorem that $M$ is an open, dense subset of the support of $V$ but as mentioned before, it is not known whether one can conclude, 
just under the stationarity hypothesis, that the singular set of $V$ must have zero $n$-dimensional Hausdorff measure. On the other hand, since $V$ is stable, a computation (assuming orientability of $M$ ) of the second variation with respect to area for compactly supported deformations of $M$ in the direction normal to $M$ shows that the stability inequality

$$
\int_{M}|A|^{2} \zeta^{2} d \mathcal{H}^{n} \leq \int_{M}|\nabla \zeta|^{2} d \mathcal{H}^{n}
$$

holds for every test function $\zeta \in C_{c}^{1}(M)$. This condition is equivalent to non-negativity of the Dirichlet eigenvalues of the Jacobi operator $\mathcal{L}$ on $M$, defined by $\mathcal{L} \zeta=\Delta \zeta+|A|^{2} \zeta$ for $u \in C^{2}(M)$. Here $A$ is the second fundamental form of $M, \nabla$ is the gradient operator on $M, \mathcal{H}^{n}$ denotes the $n$-dimensional Hausdorff measure and $\Delta$ is the Laplace-Beltrami operator on $M$. (Incidentally, as mentioned above, the orientability assumption on $M$ is not needed for the results of [Wic14a] since a weaker stability hypothesis suffices. See the statement of Theorem 3.3 below). Note that the test function $\zeta$ in the above stability inequality is required to be zero near the singular set of $V$; thus, unlike the stationarity hypothesis which requires deformation of both regular and singular parts of $V$, the stability inequality a priori imposes absolutely no restriction on the singular set.

If however we assume, as in the Schoen-Simon result, that the singular set has locally finite $(n-2)$-dimensional Hausdorff measure (or slightly more generally, that the singular set has zero 2-capacity), then an easy cut-off function argument can be used to strengthen the stability inequality a priori so that it is valid with any choice of $\zeta$ that is the restriction to $M$ of a function $\zeta_{1} \in C_{c}^{1}(U)$. Thus, under such a hypothesis on the size of the singular set, $\zeta$ only needs to vanish near $\partial U$ and no longer needs to vanish near the singular set of $V$. This is the sense in which the singular set is a priori removable for the stability inequality. Having the freedom to use, in the stability inequality, ambient test functions that behave in an arbitrary way over the singular set (whose location is unknown) was essential to the Schoen-Simon method of proof, and in particular allowed them to deduce various a priori estimates on the size of the region where $|A|$ is potentially large.

In the absence of any such smallness hypothesis on the singular set, how, or even whether, such a seemingly weak stability hypothesis could possibly control the singular set was perhaps the most fundamental question on regularity of stable hypersurfaces that had remained open since the work [SS81]. This issue was in fact just as serious even under a natural smallness hypothesis on the singular set that is more general than in [SS81], such as $\mathcal{H}^{n-1}(\operatorname{sing} V)=0$ (which, in view of examples such as pairs of crossed hyperplanes, is the weakest size hypothesis under which the conclusions of the theorem could possibly be true). For if a large singular set is present, then the amount of curvature of the regular part $M$ concentrating near the singular set could be large, causing the second fundamental form of 
$M$ to blow up on approach to the singular set even in an integral sense; indeed, with no information on the size of the singular set (or even with the assumption $\mathcal{H}^{n-1}(\operatorname{sing} V)=0$ ), it is not at all clear a priori whether stability of $M$ for normal perturbations leaving a neighborhood of $\operatorname{sing} V$ fixed implies that $A \in L^{1}(M \cap K)$ for every compact subset $K \subset U$, let alone the validity of the stability inequality across the singular set.

The work [Wic14a] develops a method (outlined in Part 2 of this article) that surmounts this basic geometric difficulty and proves claims (a), (b) for $V \in \mathcal{S}$ and the compactness theorem for $\mathcal{S}$ subject to no assumption on the singular sets beyond ruling out classical singularities; in particular, it is shown that no smallness hypothesis on the singular set of $V$ is necessary, and the sharp dimension bound on the singular set is entirely a conclusion.

This work is perhaps of interest as much for the method of proof it develops as it is for the end result. It builds upon a large body of results and ideas found in earlier fundamental work in Geometric Measure Theory, both in the regularity theory of minimal submanifolds and on the analysis of their singularities. These include the a priori estimates established in the work of Schoen-Simon [SS81] discussed above as well as results and variants of techniques developed in the work of De Giorgi $([\mathbf{D G 6 1}])$, Simons $([\mathbf{S J 6 8}])$, Federer $([$ Fed70 $])$, Allard ([All72 $])$, HardtSimon ([HS79]), Almgren ([Alm83]) and Simon ([Sim93]). In particular, the ideas contained in Simon's seminal work [Sim93] on asymptotics for minimal submanifolds near singularities play an extremely crucial role in [Wic14a]. The work also introduces some novel techniques that have since been useful in other contexts (e.g. in [Hug] and [Krum2]) and appear to hold promise for further development.

Structure of the rest of the paper. The rest of the paper consists of two parts, Part 1 and Part 2. Part 1 contains a brief survey of some (earlier) work related to the results of $[\mathbf{W i c 1 4 a}$ and a statement of the main results of [Wic14a], followed by a discussion of some applications of this work and questions arising from it.

One of our main goals in this paper is to elucidate the fact that the theory developed in [Wic14a] is quite general, and is ultimately based upon a few basic principles that are also widely in use in many other parts of Geometric Analysis. For this reason one could imagine that the methods developed in [Wic14a] may have applications in further study. With this in mind, in Part 2 we outline the proof strategy adopted in [Wic14a], focusing on how to handle the aforementioned geometric difficulties and referring the reader to [Wic14a] for details of the more technical aspects of the proof.

A more detailed guide to the rest of the paper is as follows:

Part 1 is subdivided into Sections 1-4. In Section 1 we describe the basic notion of first variation with respect to the area functional, first for smooth submanifolds and then for the larger class of integral varifolds, and discuss several consequences of stationarity, i.e. vanishing of the first 
variation. In Section 2 we give a brief discussion of singularities of stationary integral varifolds, describe two special types of singularities (namely, classical singularities and branch points), describe the key difficulties in the study of singularities of general stationary varifolds, and describe some known results on fine properties of singularities of stationary integral varifolds in a given "multiplicity 1 class". Section 3 consists of a discussion of regularity of stable minimal hypersurfaces including a variety of classical results and the recent results in [Wic14a]. Section 4 describes some applications of the results in [Wic14a]. Part 1 ends in Section 5 where some open questions arising from the work [Wic14a] are discussed.

Part 2, which is subdivided into Sections 6-8, gives an outline of the proof of the main results in [Wic14a] emphasizing geometric aspects of the proof, and highlighting the general principles and the earlier fundamental work upon which the proof is built.

It is a pleasure to thank Spencer Hughes for valuable comments on a first draft of this paper.

Part 1. Stationary varifolds, their singularities, and the COdiMENSION 1 THEORY FOR STABLE VARIFOLDS

\section{The Allard-Almgren theory of varifolds}

1.1. Smooth minimal submanifolds. Let $U$ be an open subset of $\mathbb{R}^{n+1}$. (More generally, one may consider an $(n+1)$-dimensional Riemannian manifold $U$, but since in this article we are concerned with local questions, we shall work in Euclidean space. There is little loss of generality in doing this since extending the results to more general Riemannian manifolds only requires routine technical modifications to the arguments needed for the Euclidean case.) Let $m$ be an integer with $1 \leq m \leq n$, and let $M$ be an embedded $m$-dimensional $C^{1}$ submanifold of $U$ with the closure $\bar{M}$ of $M$ in $U$ a $C^{1}$ submanifold with boundary $\partial M$ (possibly with $\partial M=\emptyset$ ), with locally finite area, i.e. with $\mathcal{H}^{m}(M \cap K)<\infty$ for each compact subset $K$ of $U$, where $\mathcal{H}^{m}$ denoted the $m$-dimensional Hausdorff measure. $M$ is said to be stationary in $U$ if $M$ has zero first variation with respect to the area functional for all compactly supported ambient deformations. This means precisely that

$$
\left.\delta M(\psi) \equiv \frac{d}{d t}\right|_{t=0} \mathcal{H}^{n}\left(\varphi_{\psi, t}(M)\right)=0
$$

for every ambient vector field $\psi \in C_{c}^{1}\left(U ; \mathbb{R}^{n+1}\right)$, where $\varphi_{\psi, t}(X)=X+$ $t \psi(X)$ for $(t, X) \in(-\epsilon, \epsilon) \times U$ with $\epsilon>0$ sufficiently small. A computation using the area formula $([\mathbf{S i m 8 3 a}]$, Sec. 9) shows that $\delta M(\psi)=$ 
$\int_{M} \operatorname{div}_{M} \psi(X) d \mathcal{H}^{n}(X)$, and consequently, stationarity of $M$ in $U$ is equivalent to the requirement that

$$
\int_{M} \operatorname{div}_{M} \psi(X) d \mathcal{H}^{n}(X)=0 \quad \forall \psi \in C_{c}^{1}\left(U ; \mathbb{R}^{n+1}\right) .
$$

Here $\operatorname{div}_{M} \psi(X)=\sum_{j=1}^{n} \tau_{j} \cdot D_{\tau_{j}} \psi(X)$, where $\left\{\tau_{1}, \tau_{2}, \ldots, \tau_{n}\right\}$ is any orthonormal basis for the tangent space $T_{X} M$ and $D_{\tau}$ denotes the directional derivative in the direction $\tau$.

The preceding equation is known as the first variation formula. One can use this equation and the quasilinear elliptic PDE theory to establish higher regularity (in fact analyticity) of $M$ away from $\partial M \cup \partial U$. It then follows from the Divergence Theorem that stationarity of $M$ in $U$ is also equivalent to the fact that $\partial M=\emptyset$ and $M$ has zero mean curvature everywhere, i.e. that $M$ is a classical minimal submanifold of $U$ with no boundary in $U$.

1.2. Varifolds. For various natural reasons such as for establishing existence of $m$-dimensional minimal submanifolds in a compact Riemannian manifold $N$ for fixed $m<\operatorname{dim} N$ (see [Alm65], [Pit77], [SS81]), for understanding the completion of the space of mass bounded smooth minimal submanifolds of a given dimension $m$, and for analyzing, by studying tangent cones, the nature of singularities that may arise in various situations including in carrying out either of the preceding tasks, it is highly desirable to work in a space $\mathcal{V}$ of "possibly singular $m$-dimensional surfaces." Such a space must obviously contain the set of $C^{1}$ submanifolds with locally finite area, and must admit (a) an extension of the area functional, (b) an extension of the above variational definition of stationarity and (c) a topology relative to which the area functional, at least in the case of compact ambient manifolds, is continuous, and the stationary elements form a closed subset on which the area functional is always continuous. With these properties of the space $\mathcal{V}$, the existence question may be approached via min-max methods and singularity analysis will be facilitated by tangent cones being non-trivial and stationary. (Note that direct area minimization - for which area lower semi-continuity would suffice - is not possible as a way of proving existence unless the surfaces are constrained in some way such as by a boundary value requirement or non-trivial ambient space homology).

A natural class of surfaces satisfying requirements (a) and (b) is the set of $m$-rectifiable subsets $M$, with area equal to the $m$-dimensional Hausdorff measure; indeed, the above first variation formula makes sense for such $M$, and in the case of Euclidean ambient space, is equivalent (by the same computation as in the case of $C^{1}$ submanifolds) to the same variational requirement that $\delta(M)(\psi)=0$ for each $C^{1}$ ambient vector field $\psi$ with compact support. The requirement (c) however is more subtle. It forces one to enlarge the class even more, to consist of $m$-dimensional integral varifolds (which we shall often refer to as integral $m$-varifolds below), a class of measure-theoretic $m$-dimensional surfaces with the topology of measure theoretic convergence. This space was introduced by Almgren in his 
seminal paper [Alm65] where he established among other things the crucial closure theorem for elements satisfying appropriate variational requirements, which in particular implied the closure property for the stationary elements (Theorem 1.1 below). The definition and properties of this class were streamlined by Allard in his landmark paper [All72] in which he generalized Almgren's closure theorem and also established a fundamental regularity theorem which in particular applies to stationary elements (see Theorem 1.2 below).

We now define this class, and in Sections 1.3-1.8 below extend the notion of stationarity to it and discuss briefly the main consequences of stationarity. We remark that although our discussion here is restricted to the stationary case, much of the theory is valid in a more general setting, for instance for integral varifolds with locally bounded first variation whose first variation measure has no singular part and generalized mean curvature satisfies suitable local integrability hypotheses; stationarity corresponds to the case when the generalized mean curvature is zero. Besides [All72], a standard reference for the theory of varifolds in full generality is [ $[\mathbf{S i m 8 3 a}$ ], Chapters 4 and 8], to which we shall refer the reader for details of various assertions made below.

Let $U$ be open in $\mathbb{R}^{n+1}$ as before, $m$ an integer with $1 \leq m \leq n$, and let $V$ be an $m$-dimensional varifold (or an $m$-varifold for short) on $U$. Thus $V$ is a non-negative Radon measure on $U \times G(m, n)$, where $G(m, n)$ is the space of $m$-dimensional subspaces of $\mathbb{R}^{n+1}$ (with the topology induced by any of the equivalent norms).

Any $m$-varifold $V$ on $U$ naturally induces a Radon measure on $U$, called the weight measure associated with $V$, denoted $\|V\|\left(\mu_{V}\right.$ in [Sim83a]) and defined by $\|V\|(A)=V(A \times G(m, n))$ for $A \subset U$.

The varifold $V$ is integral if for $\|V\|$-a.e. $X \in U$, there is an $m$ dimensional plane $T=T_{X} V$ (the tangent space of $V$ ) and a positive integer $\theta=\theta_{V}(X)$ (the multiplicity of $V$ at $X$ ) such that $\lim _{\lambda \rightarrow 0^{+}} V_{X, \lambda}=\theta|T|$ where the convergence is as Radon measures on $\mathbb{R}^{n+1} \times G(m, n)$,

$$
\begin{gathered}
V_{X, \lambda}(A)=\lambda^{-m} V(\{(X+\lambda Y, S):(Y, S) \in A\} \cap(U \times G(m, n))) \quad \text { and } \\
|T|(A)=\mathcal{H}^{m}(T \cap\{Y:(Y, T) \in A\})
\end{gathered}
$$

for $A \subset \mathbb{R}^{n+1} \times G(m, n)$. (See $[$ Sim83a], Sec. 38.1.)

Any countably $m$-rectifiable subset $M$ of $U$ with locally $\mathcal{H}^{m}$ integrable, positive integer-valued multiplicity function $\theta_{M}$ (see [Sim83a], Sec. 11) defines an integral $m$-varifold $|M|$ on $U$ via

$$
|M|(A)=\int_{\left\{X:\left(X, T_{X} M\right) \in A\right\}} \theta_{M}(X) d \mathcal{H}^{m}(X)
$$

for $A \subset U \times G(m, n)$. The converse of this is also true; i.e. if $V$ is an integral $m$-varifold on $U$, then the set $M$ of points $X \in U$ where $V$ has an $m$ dimensional tangent plane $T_{X} V$ with positive integer multiplicity $\theta_{V}(X)$ is 
countably $m$-rectifiable, $\theta_{V}$ is locally $\mathcal{H}^{m}$ integrable on $M$ and $V=|M|$ if we take $\theta_{M}=\theta_{V}$. (See [Sim83a], Theorem 38.3.)

In particular, an $m$-dimensional $C^{1}$ submanifold $M$ of $U$ with locally finite mass (i.e. locally finite $m$-dimensional Hausdorff measure) defines the integral $m$-varifold $|M|$ (with multiplicity 1 ) given by

$$
|M|(A)=\mathcal{H}^{m}\left(M \cap\left\{X:\left(X, T_{X} M\right) \in A\right\}\right)
$$

for $A \subset U \times G(m, n)$.

Let $\operatorname{IV}_{m}(U)$ denote the space of integral $m$-varifolds on $U$.

We define the regular set $\operatorname{reg} V$ of an $m$-varifold $V$ on $U$ to be the set of points $X \in \operatorname{spt}\|V\| \cap U$ such that there exists $\rho \in(0$, dist $(X, \partial U))$ with the property that spt $\|V\| \cap \operatorname{clos} B_{\rho}^{n+1}(X)$ is a smooth, embedded, compact, connected $m$-dimensional submanifold with boundary $\subset \partial B_{\rho}^{n+1}(X)$. Here and subsequently, $B_{\rho}^{n+1}(X)$ denotes the open ball in $\mathbb{R}^{n+1}$ with center $X$ and radius $\rho$, and clos denotes the closure in $\mathbb{R}^{n+1}$.

The singular set $\operatorname{sing} V$ is the set of points $X \in \operatorname{spt}\|V\| \cap U$ with $X \notin \operatorname{reg} V$.

1.3. First variation of a varifold and stationarity. The first variation of an $m$-varifold $V$ on $U$ with respect to the area functional is the map $\delta V: C_{c}^{1}\left(U ; \mathbb{R}^{n+1}\right) \rightarrow \mathbb{R}$ given by

$$
\delta V(\psi)=\left.\frac{d}{d t}\right|_{t=0}\left\|\varphi_{\psi, t \#} V\right\|(U)
$$

where for a given vector field $\psi \in C_{c}^{1}\left(U ; \mathbb{R}^{n+1}\right), \varphi_{\psi, t}(\cdot)$ is the map defined in Section $1.1\left(\varphi_{\psi, t}(X)=X+t \psi(X)\right.$ for $t$ sufficiently small $)$, and $\varphi_{\psi, t \#}$ is the mapping induced on the space of $m$-varifolds on $U$ by $\varphi_{\psi, t}$ (see [[Sim83a], Sec. 39]). By the same computation as in the case of $m$-dimensional $C^{1}$ submanifolds, we then have the first variation formula which tells us that

$$
\delta V(\psi)=\int_{U \times G(m, n)} \operatorname{div}_{S} \psi(X) d V(X, S)
$$

for every $\psi \in C_{c}^{1}\left(U ; \mathbb{R}^{n+1}\right)$. Here $\operatorname{div}_{S} \psi=\sum_{j=1}^{m} \tau_{j} \cdot D_{\tau_{j}} \psi$ where $\left\{\tau_{1}, \ldots, \tau_{m}\right\}$ is any orthonormal basis for $S$, or, alternatively, $\operatorname{div}_{S} \psi=\sum_{j=1}^{n+1} e_{j} \cdot p_{S} D \psi^{j}$, where $\left\{e_{1}, e_{2}, \ldots, e_{n+1}\right\}$ is any orthonormal basis for $\mathbb{R}^{n+1}, \psi^{j}=e_{j} \cdot \psi$ for $j=1,2, \ldots, n+1, D$ denotes the gradient operator on $U$ and for $S \in G(m, n), p_{S}$ denotes the orthogonal projection of $\mathbb{R}^{n+1}$ onto $S$.

$V$ is stationary in $U$ if

$$
\delta V(\psi)=0 \quad \text { for each } \quad \psi \in C_{c}^{1}\left(U ; \mathbb{R}^{n+1}\right) .
$$

1.4. The monotonicity formula. Let $m, n, U$ be as above, and let $V$ be an $m$-varifold on $U$. Suppose that $V$ is stationary in $U$ and let $Y \in U$. By taking $\psi(X)=(X-Y) \zeta\left(\rho^{-1}|X-Y|\right)$ in the identity (1.3) where, for some $\tau \in(0,1), \zeta \in C^{1}(\mathbb{R})$ with $\zeta(t)=1$ for $t \leq \tau, \zeta(t)=0$ for $t \geq 1$ and 
$\zeta^{\prime}(t) \leq 0$, and letting $\tau \rightarrow 1$, we deduce (see [[Sim83a], Secs. $\left.17 \& 40\right]$ ) the identity

$$
\frac{d}{d \rho}\left(\rho^{-m}\|V\|\left(B_{\rho}^{n+1}(Y)\right)\right)=\rho^{2-m} \int_{\partial B_{\rho}^{n+1}(y)} \frac{\left|D^{\perp}(X-Y)\right|^{2}}{|X-Y|^{m}} d\|V\|(X)
$$

for a.e. $\rho \in(0, \operatorname{dist}(x, \partial U))$; in particular the function

$$
\rho \mapsto \rho^{-m}\|V\|\left(B_{\rho}^{n+1}(Y)\right)
$$

is monotone non-deceasing for $\rho \in(0$, dist $(x, \partial U))$ and hence the density

$$
\Theta(\|V\|, Y)=\lim _{\rho \rightarrow 0^{+}}\left(\omega_{n} \rho^{m}\right)^{-1}\|V\|\left(B_{\rho}^{n+1}(Y)\right)
$$

of $V$ at $Y$ exists, and is a non-negative real number, for every $Y \in U$.

Furthermore, upon integration, the preceding differential identity yields the monotonicity formula

$$
\begin{aligned}
& \left(\omega_{m} \rho^{m}\right)^{-1}\|V\|\left(B_{\rho}^{n+1}(Y)\right)-\Theta(\|V\|, Y) \\
& =\omega_{m}^{-1} \int_{B_{\rho}^{n+1}(Y)} \frac{\left|D^{\perp}(Y-X)\right|^{2}}{|X-Y|^{n}} d\|V\|(X),
\end{aligned}
$$

valid for each $Y \in U$ and $\rho \in(0$, dist $(Y, \partial U))$. This simple consequence of stationarity plays an extremely crucial role in the regularity and singularity theories of stationary varifolds.

1.5. The Allard-Almgren Compactness Theorem. If $V_{j}$ is an $m$ varifold on $U$ for $j=1,2,3, \ldots$ with

$$
\limsup _{j \rightarrow \infty}\left\|V_{j}\right\|(K)<\infty
$$

for each compact set $K \subset U$, then it is a direct consequence of the BanachAlaoglu theorem that there exists a varifold $V$ on $U$ and a subsequence $\left\{j^{\prime}\right\}$ of $\{j\}$ such that $V_{j^{\prime}}$ converges to $V$ as varifolds (i.e. as Radon measures on $U \times G(m, n)$ in the weak ${ }^{\star}$ topology); a very important, more subtle result, which follows from the Allard-Almgren integer varifold compactness theorem ([Alm65], [All72]; see also [[Sim83a], Theorem 42.7]), is the following, which guarantees both stationarity and integrality of the limit varifold $V$ whenever the $V_{j}$ are integral and stationary:

Theorem 1.1 (Almgren ([Alm65]); Allrad ([All72] $])$. Let $V_{j} \in I V_{m}(U)$ be as above, with $V_{j}$ stationary in $U$. Then there exists a subsequence $\left\{V_{j^{\prime}}\right\}$ of $\left\{V_{j}\right\}$ and a varifold $V \in I V_{m}(U)$ stationary in $U$ such that $V_{j^{\prime}} \rightarrow V$ as varifolds on $U$.

1.6. Upper semi-continuity of density. The monotonicity of the function

$$
\rho \mapsto \rho^{-m}\|V\|\left(B_{\rho}^{n+1}(Y)\right), \quad \rho \in(0, \operatorname{dist}(Y, \partial U)),
$$

whenever $Y \in U$ and $V$ is a stationary $m$-varifold on $U$ implies that the density function $(V, Y) \mapsto \Theta(\|V\|, Y)$ is upper-semicontinuous, i.e. if 
$Y_{j}, Y \in U$ for $j=1,2,3, \ldots$ with $Y_{j} \rightarrow Y$, and $V_{j}, V$ are stationary $m$ varifolds on $U$ with $V_{j} \rightarrow V$, then

$$
\Theta(\|V\|, Y) \geq \limsup _{j \rightarrow \infty} \Theta\left(\left\|V_{j}\right\|, Y_{j}\right) .
$$

This in particular implies that if $V \in \operatorname{IV}_{m}(U)$ is stationary, then

$$
Y \in \operatorname{spt}\|V\| \cap U \Longleftrightarrow \Theta(\|V\|, Y) \geq 1 \text {. }
$$

1.7. Tangent cones and the question of their uniqueness. An important consequence of the monotonicity formula (1.4) and the integer varifold compactness theorem (Theorem 1.1) is the existence of non-trivial tangent cones to any stationary $V \in \operatorname{IV}_{m}(U)$ at every point in spt $\|V\|$. More precisely, if $V \in \operatorname{IV}_{m}(U)$ is stationary then corresponding to each $Y \in$ spt $\|V\| \cap U$ and each sequence of positive numbers $\left\{\rho_{j}\right\}$ with $\rho_{j} \rightarrow 0$ as $j \rightarrow \infty$, there exists a subsequence $\left\{\rho_{j^{\prime}}\right\}$ of $\left\{\rho_{j}\right\}$ and a non-trivial cone $\mathbf{C}$, i.e. $\mathbf{C} \in \operatorname{IV}_{m}\left(\mathbb{R}^{n+1}\right) \backslash\{0\}$ with $\eta_{0, \rho \#} \mathbf{C}=\mathbf{C}$ for each $\rho>0$, such that $\mathbf{C}$ is stationary in $\mathbb{R}^{n+1}$ and

$$
\eta_{Y, \rho_{j^{\prime}} \#} V \rightarrow \mathbf{C}
$$

as $j^{\prime} \rightarrow \infty$, where the convergence is as varifolds on $\mathbb{R}^{n+1}$ ([[Sim83a], Sec. $42])$. Here and subsequently, for $Y \in \mathbb{R}^{n+1}$ and $\rho>0, \eta_{Y, \rho}: \mathbb{R}^{n+1} \rightarrow \mathbb{R}^{n+1}$ is the map defined by

$$
\eta_{Y, \rho}(X)=\frac{X-Y}{\rho}
$$

We also have that

$$
\Theta(\|V\|, Y)=\Theta(\|\mathbf{C}\|, 0)=\left(\omega_{m} \rho^{n}\right)^{-1}\|\mathbf{C}\|\left(B_{\rho}(0)\right)
$$

for each $\rho>0$.

If $V \in \operatorname{IV}_{m}(U)$ is stationary in $U$ and $Y$ is a point in spt $\|V\| \cap U$ where the tangent plane $T_{Y} V$ exists (as it does for $\|V\|$-a.e. $Y \in$ spt $\|V\|$ ), it follows from Theorem 1.1 and the first variation formula (1.3) that $\Theta(\|V\|, Y)$ is a positive integer and $T_{Y} V$ with constant multiplicity equal to $\Theta(\|V\|, Y)$ is the unique tangent cone to $V$ at $Y$. In particular, this is the case when $Y \in \operatorname{reg} V$. When $Y$ is an arbitrary point in $\operatorname{sing} V$ however, uniqueness of tangent cone $\mathbf{C}$ at $Y$, i.e. the question whether $\mathbf{C}$ is independent of the sequence $\left\{\rho_{j}\right\}$, remains a major open question. (Examples of stationary rectifiable but non-integral varifolds with non-unique tangent cones have recently been constructed by Kolár $([\mathbf{J K}])$.)

We shall denote by $\operatorname{VarTan}(V, Y)$ the set of all tangent cones to $V$ at $Y \in \operatorname{spt}\|V\| \cap U$.

The answer to the above uniqueness question concerning tangent cones C to stationary integral $m$-varifolds $V$ at singular points is known in the affirmative in a few special cases including the following:

(i) when $m=1$, i.e. when $V$ is 1-dimensional ([AA 76] $)$; 
(ii) when $\mathbf{C}$ is a multiplicity 1 cone with an isolated singularly, i.e when $\Theta(\|\mathbf{C}\|, X)=1$ for each $X \in \operatorname{spt}\|\mathbf{C}\| \backslash\{0\}$ and $\operatorname{sing} \mathbf{C}=\{0\}$ ( $[$ Sim83b]; see Theorem 2.2 below);

(iii) when $\mathbf{C}=\mathbf{C}_{0} \times L$ where $\mathbf{C}_{0}$ is the 1-dimensional multiplicity 1 stationary varifold corresponding to three half-lines meeting at a point and $L$ is an $(m-1)$-dimensional subspace ([Sim93]);

(iv) when $V$ corresponds to a current in a codimension 1 multiplicity 1 class of currents (see Section 2.3 below) and $\mathbf{C}=\mathbf{C}_{0} \times L$ where $L$ is a 1-dimensional subspace, $\mathbf{C}_{0}$ is an $(m-1)$-dimensional multiplicity 1 strictly minimizing, strictly stable cone (in the sense described in [HS85]) with an isolated singularity and $\mathbf{C}_{0}$ satisfies a certain integrability condition on its Jacobi fields and an eigenvalue condition on its Jacobi field operator ([Sim94]). These conditions are satisfied by all codimension 1 area minimizing cones $\mathbf{C}_{0}$ whose intersection $\Sigma$ with $\mathbb{S}^{m-1}$ is a product of spheres except when $\Sigma$ is $\frac{1}{\sqrt{2}} \mathbb{S}^{3} \times \frac{1}{\sqrt{2}} \mathbb{S}^{3}$ or $\frac{1}{\sqrt{3}} \mathbb{S}^{2} \times \frac{\sqrt{2}}{\sqrt{3}} \mathbb{S}^{4}$

(v) when $V$ corresponds to a two dimensional area minimizing rectifiable current with no boundary ([Whi83]);

(vi) when $V$ corresponds to a stable codimension 1 integral current with no boundary and $\mathbf{C}$ is either a multiplicity 2 hyperplane or a multiplicity 1 pair of transversely intersecting hyperplanes ([Wic] $)$;

(vii) when $V$ corresponds to certain semi-calibrated currents (which in some cases are stationary) ([CB14-a], [CB14-b]);

(viii) when $V$ corresponds to a Lipschitz 2-valued graph over a domain in $\mathbb{R}^{m}$, and $\mathbf{C}$ corresponds to a union of four distinct half-planes meeting along a common $(m-1)$-dimensional axis (which in particular includes the case of a union of two transverse planes) ([Hug $]$ ).

1.8. Interior regularity of stationary varifolds: Allard's theorems. The celebrated regularity theorem of Allard ([All72], [[Sim83a], Theorem 23.1]) implies the following:

TheOrem 1.2 (Allard $([\mathbf{A l l 7 2}]))$. For each given $\alpha \in(0,1)$, there exists a constant $\epsilon=\epsilon(n, m, \alpha)>0$ such that if $V \in \operatorname{IV}_{m}\left(B_{R}^{n+1}(Y)\right)$ is stationary in $B_{R}^{n+1}(Y), Y \in \operatorname{spt}\|V\|$,

$$
\begin{gathered}
\left(\omega_{n} R^{n}\right)^{-1}\|V\|\left(B_{R}^{n+1}(Y)\right)<2-\alpha \quad \text { and } \\
R^{-n-2} \int_{B_{R}^{n+1}(Y)} \operatorname{dist}^{2}(X, P) d\|V\|(X)<\epsilon
\end{gathered}
$$

for some $m$-dimensional affine plane $P$ with $Y \in P$, then spt $\|V\| \cap$ $B_{R / 2}^{n+1}(Y) \subset \operatorname{reg} V$. In fact spt $\|V\| \cap B_{R / 2}^{n+1}(Y) \subset \operatorname{graph} u \subset \operatorname{reg} V$ where $u: P \cap B_{R / 2}^{n+1}(Y) \rightarrow P^{\perp}$ is of class $C^{1, \mu}$ for some $\mu=\mu(n, m, \alpha) \in(0,1)$ 
and satisfies the estimate

$$
\begin{aligned}
& R^{-1} \sup _{P \cap B_{R / 2}^{n+1}(Y)}|u|+\sup _{P \cap B_{R / 2}^{n+1}(Y)}|D u| \\
& +R^{\mu} \sup _{X_{1}, X_{2} \in P \cap B_{R / 2}^{n+1}(Y), X_{1} \neq X_{2}} \frac{\left|D u\left(X_{1}\right)-D u\left(X_{2}\right)\right|}{\left|X_{1}-X_{2}\right|^{\mu}} \\
& \quad \leq C\left(R^{-n-2} \int_{B_{R}(Y)} \operatorname{dist}^{2}(X, P) d\|V\|(X)\right)^{1 / 2}
\end{aligned}
$$

where $C=C(n, m, \alpha) \in(0, \infty)$. In particular, any point $Y \in \operatorname{spt}\|V\|$ at which $V$ has a tangent cone equal to an m-dimensional multiplicity 1 plane is a regular point of $V$.

Remark: Since $u$ is a weak solution to the minimal surface system on the domain $P \cap B_{R / 2}^{n+1}(Y)$, it follows from elliptic PDE theory that $u$ is smooth (in fact real analytic) and that for each $k=1,2,3, \ldots$,

$$
R^{k-1} \sup _{P \cap B_{R / 2}(Y)}\left|D^{k} u\right| \leq C\left(R^{-n-2} \int_{B_{R}(Y)} \operatorname{dist}^{2}(X, P) d\|V\|(X)\right)^{1 / 2}
$$

for some constant $C=(n, m, \alpha, k) \in(0, \infty)$.

An equivalent version of the the above theorem, also due to Allard ([All72], [[Sim83a], Theorem 24.2]), asserts the following:

Theorem 1.2' (Allard $([$ All72 $]))$. There exists a constant $\epsilon_{1}=\epsilon_{1}(n, m) \in$ $(0,1)$ such that if $V \in \operatorname{IV}_{m}(U)$ is stationary in $U, Y \in \operatorname{spt}\|V\| \cap U$ and $\Theta(\|V\|, Y)<1+\epsilon_{1}$, then $Y \in \operatorname{reg} V$.

Establishing equivalence of the statements in these two theorems is fairly straightforward, with the arguments in both directions relying heavily on the monotonicity formula. A simple argument based on the monotonicity formula shows (see [[Sim83a], Theorem 24.2]) that one can choose $\epsilon_{1}=$ $\epsilon_{1}(n, m)$ such that under the hypotheses of Theorem $1.2^{\prime}$, the varifold suitably rescaled near $Y$ is weak close to a multiplicity 1 plane. Conversely, it is not difficult to see that one can choose $\epsilon=\epsilon(n, m, \alpha)$ such that under the hypotheses of Theorem 1.2, the destiny function of $V$ is uniformly close to 1 in spt $\|V\| \cap B_{3 R / 4}^{n+1}(Y)$ and hence by Theorem $1.2^{\prime}, V$ is regular in $B_{R / 2}^{n+1}(Y)$; the estimate in Theorem 1.2 (in fact a bound on the norm of the second fundamental form) can then be obtained, by showing that a sequence of smooth minimal submanifolds with mass ratios uniformly bounded by some number $\Lambda$ and second fundamental form blowing up along a sequence of points can be rescaled by the norm of the second fundamental form at appropriately chosen points to produce a complete, smooth submanifold $M$ stationary in all of $\mathbb{R}^{n+1}$, with density at infinity not larger then $\Lambda$, and with the norm of the second fundamental form at one point equal to 1 . Such $M$ by the monotonicity formula can easily be seen not to exist in case $\Lambda$ is not much larger than 1. 
It is an easy corollary of Theorem $1.2^{\prime}$ and the upper semi-continuity of the density function $\Theta(\|V\|, \cdot)$ that if $V \in \operatorname{IV}_{m}(U)$ is stationary in $U$, then $\operatorname{reg} V$ is non-empty, or, equivalently $\operatorname{reg} V$ is a dense (open) subset of spt $\|V\| \cap U$. This is the best known general regularity result for stationary integral varifolds of dimension $\geq 2$. In particular, it remains a fundamental open question in case $m \geq 2$ whether $\mathcal{H}^{m}(\operatorname{sing} V)=0$ whenever $V \in$ $\operatorname{IV}_{m}(U)$ is stationary in $U$. This remains open even in the case $m=n=2$. If $m=1$ then $\operatorname{sing} V$ consists of isolated points ([AA 76]) and spt $\|V\|$ is locally the union of finitely many straight line segments meeting at a point, or geodesic arcs meeting smoothly at a point in the case of arbitrary smooth Riemannian ambient spaces.

In remarkable recent work [Men13], Menne has established $C^{2}$ rectifiability of a very general class of integral $m$-varifolds including stationary ones, generalizing earlier similar results of Schätzle ([Sch04]) for codimension 1 integral varifolds satisfying stronger hypotheses on the first variation.

\section{Singularities of stationary varifolds}

Often in solutions to non-linear problems, and in particular in stationary varifolds, singularities are unavoidable. Therefore studying the nature of the singularities and the behavior of the solutions in the vicinity of their singularities is a central part of the analysis of the solutions. Moreover, understanding the completion of a space of smooth solutions and identifying compact subclasses of solutions also often require knowledge of the nature of singularities. However, singularity analysis remains one of the largely open challenges in modern Geometric Analysis. There has been remarkable progress in some cases, and with regard to stationary varifolds, among the known results in this direction are:

(i) sharp dimension bounds on the interior singular sets of varifolds belonging to some closed subclasses of stationary integral varifolds. These subclasses include the varifolds corresponding to area minimizing rectifiable currents both in codimension 1 (combined work [DG61], [FF60], [SJ68], [Fed70]; see Theorem 3.2 below) and in higher codimension ([Alm83]); stable codimension 1 integral varifolds with no "classical singularities" ([Wic14a]; see Section 2.2 and Theorem 3.3 below - this class in fact subsumes the class of codimension 1 area minimizers); certain stable codimension 1 integral varifolds admitting classical singularities (and hence also branch points, see Section 2.2 below) ([SimWic], [Wic]);

(ii) regularity properties of the singular sets and the asymptotic behavior on approach to the singular sets of varifolds belonging to certain subclasses of stationary integral varifolds. These include one dimensional stationary integral varifolds ([AA76]); two dimensional area minimizing currents ([SC88 $]$ ); two dimensional area minimizing flat chains $\bmod 3$ in $\mathbb{R}^{3}$ and two dimensional "soap film like" 
surfaces in $\mathbb{R}^{3}$ ([Tay73], [Tay76]); solutions to certain classes of Plateau-type problems ([Whi85]); general "multiplicity 1 classes" of stationary varifolds of any dimension and codimension ([Sim93], [Sim95]; see Section 2.3 below) and, stable codimension 1 rectifiable currents near points of density $<3$ ([KW2], [Wic $]$ ).

The questions concerning the local structure of the singular sets and asymptotics near singularities (as in (ii) above) are generally considerably more subtle than the question of the Hausdorff dimension of the singular sets, although establishing sharp dimension bounds, or, for that matter, even showing almost everywhere regularity, remains a difficult unsolved problem in several important cases; these include stable hypersurfaces admitting multiplicity $\geq 3$, stable varifolds in higher codimension (even subject to the the condition that multiplicity is $<3$ ) and of course the case of the largest class, namely stationary integral varifolds (even those of dimension 2 and codimension 1).

In this section and the next, we explain the key difficulties in general with regard to these questions, and discuss in more detail a sample of the known results referred to in (i) and (ii) above.

2.1. The Almgren-Federer Generalized Stratification. The key difficulty in estimating the dimension of the singular set of a stationary integral varifold of dimension $m \geq 2$ stems from the presence of higher multiplicity; more precisely, the difficulty is in controlling the size of the set of those singularities (branch points, see Section 2.2 below) at which there is at least one tangent cone with support an $m$-dimensional plane and multiplicity a constant integer (which by Allard's regularity theorem must be $\geq 2$ ). All other singularities form a set of Hausdorff dimension $\leq m-1$, a fact that follows from the case $j=m-1$ of the following general result:

Theorem 2.1 (Almgren-Federer Generalized Stratification; [Alm83]). Let $V \in \mathrm{IV}_{m}(U)$ be stationary in $U$, and recall that $\operatorname{VarTan}(V, X)$ denotes the set of tangent cones to $V$ at $X \in \mathrm{spt}\|V\| \cap U$. For a given cone $\mathbf{C}$ in $\mathbb{R}^{n+1}$, let $S(\mathbf{C})$ denote the linear subspace of $\mathbb{R}^{n+1}$ of directions along which spt $\|\mathbf{C}\|$ is translation invariant. Then for each $j=0,1,2, \ldots, m$,

$$
\operatorname{dim}_{\mathcal{H}}(\{X \in \operatorname{sing} V: \operatorname{dim} S(\mathbf{C}) \leq j \forall \mathbf{C} \in \operatorname{VarTan}(V, X)\}) \leq j .
$$

Federer first proved a special case of this theorem ([Fed70]; see also [[Sim83a], Appendix A]) and applied it to area minimizing hypersurfaces. The theorem in the generality given above is due to Almgren ([Alm83]) who deduced it by a clever argument as an elementary consequence of the upper semi-continuity of the density function $(V, Y) \mapsto \Theta(\|V\|, Y)$ and the fact that $\Theta(\|\mathbf{C}\|, 0)=\Theta(\|V\|, Y)$ for each $\mathbf{C} \in \operatorname{VarTan}(V, Y)$.

Despite its elementary nature, this theorem has been extremely useful in a variety of situations, including in the regularity theory for stable codimension 1 varifolds ([Wic14a]); see the discussion in Section 6 below. Being a consequence of very general principles, it holds in appropriate 
forms in various other contexts as well. For instance, Schoen and Uhlenbeck adapted it in [SU82] to the setting of energy minimizing maps between Riemannian manifolds; see [Sim96] for a nice, concise presentation of its proof following [Alm83] in that context. In [Whi97], White established an elegant version of it for integral Brakke flows.

2.2. Classical singularities and branch points. It is convenient at this point to interrupt our discussion of the general singularity theory to introduce the following two special types of singularities, which we shall refer to heavily later in the article, in particular in the context of stable codimension 1 integral varifolds.

Let $V \in \operatorname{IV}_{m}(U)$.

(a) Classical singularities: A point $Z \in \operatorname{sing} V$ is said to be a classical singularity of $V$ if there exists $\rho>0$ and $\alpha \in(0,1)$ such that spt $\|V\| \cap B_{\rho}(Y)$ is the finite union of three or more embedded, $m$-dimensional $C^{1, \alpha}$ submanifoldswith-boundary of $B_{\rho}(Y)$, having a common $(m-1)$-dimensional $C^{1, \alpha}$ (free) boundary $\Gamma$ containing $Y$, and meeting only along $\Gamma$.

(b) Branch points: A point $Z \in \operatorname{sing} V$ is said to be a branch point of $V$ if: (i) there is a tangent cone to $V$ at $Z$ with support an $m$-dimensional plane and (ii) near $Z$, spt $\|V\|$ is not the union of (finitely many) smoothly embedded $m$-dimensional submanifolds.

Remarks: (1) In case $V$ is stationary and $m=n$ (i.e. when $V$ has codimension 1), in the definition of classical singularity, the requirement of $C^{1, \alpha}$ regularity up to $\Gamma$ can be replaced by real analyticity up to $\Gamma$ (in the case of analytic ambient spaces) or smoothness up to $\Gamma$ (in the case of smooth ambient spaces). This was shown by Kinderlehrer-Nirenberg-Spruck ([KNS78]) using a PDE theoretic argument in the case when the number of sheets of spt $\|V\| \cap B_{\rho}(Y)$ is three, and extended recently to the general case of an arbitrary number of sheets by Krummel ([Krum2]) whose proof combines the main idea of [KNS78] with a more geometric view point.

(2) If $Z \in \operatorname{sing} V$ is a classical singularity, then of course $V$ has a unique tangent cone at $Z$ whose support is the union of three or more $m$-dimensional half-planes meeting along a common $(m-1)$-dimensional axis. The converse to this is not true in general, but is known to be true in some interesting special cases. For instance: (i) whenever $V$ is stationary and $\Theta(\|V\|, Z)=$ $3 / 2$ ([Sim93]); (ii) whenever $V$ is a stationary hypersurface corresponding to a rectifiable current with no boundary and the regular part of $V$ is stable (see definition 3.1) and $\Theta(\|V\|, Z)=2([\mathbf{W i c}])$ and (iii) whenever $V$ is the graph of a two-valued Lipschitz function $u$ over a domain in $\mathbb{R}^{m}$ taking values in the space of unordered pairs of elements in $\mathbb{R}^{n+1-m}$ with $n>m$, and $\mathbf{C}$ is the multiplicity 1 cone supported on four distinct $m$-dimensional half-planes meeting along a common axis, but not forming a pair of $m$ dimensional planes $([\mathbf{H u g}])$.

Examples: (a) Area minimizers: If $V$ is the varifold corresponding to an area minimizing rectifiable current with no boundary, then straightforward 
comparison surface constructions show that $V$ has no classical singularities. It follows from De Giorgi's work [DG61] that such area minimizers of codimension 1 do not carry branch point singularities. In codimension $\geq 2$ on the other hand branch points exist in area minimizers, and a large family of concrete examples is provided by complex analytic varieties with branch points (e.g. $M=\left\{(z, w): z^{2}=w^{3}\right\} \cap \mathbb{C} \times \mathbb{C}$ ) which are all calibrated and hence locally area minimizing.

(b) Stable varifolds: Both classical singularities and branch points exist in general in stable varifolds (see definition 3.1) in any dimension $\geq 2$ and codimension. A pair of crossed hyperplanes is an obvious example of a stable varifold with classical singularities, and in general, any stationary codimension 1 varifold in a sufficiently small neighborhood about a classical singularity is stable (in the sense of definition 3.1).

As for stable varifolds with branch points, since any area minimizing surface is stable, there are plenty of such examples in codimension $\geq 2$. Unlike in the case of area minimizers, they also exist in codimension 1. Simon and the author ([SimWic07] $)$ constructed a large family of such examples as multi-valued codimension 1 graphs over the cylinder $B_{1}^{2}(0) \times \mathbb{R}^{n-2}$ with prescribed multi-valued boundary data satisfying a mild symmetry condition. Rosales [Ros10] constructed a different family of two dimensional branched surfaces using a variant of the method introduced in [SimWic07], and more recently, Krummel [Krum1] gave a direct PDE theoretic approach to the results in [SimWic07], also extending the construction to higher codimension under an additional necessary smallness hypothesis on the boundary data.

A highly nontrivial fact is that in a stable codimension 1 varifold, branch points cannot exist without classical singularities nearby ([Wic14a]); that is to say, every branch point is the limit point of classical singularities. This follows from Theorem 6.1 below, which we shall discuss in detail in Part 2. This statement is of course false in higher codimension, as shown by the locally area minimizing example $M=\left\{(z, w): z^{2}=w^{3}\right\} \cap \mathbb{C} \times \mathbb{C}$ in which the only singularity is the branch point at the origin.

(c) Stationary varifolds: All of the examples discussed above are of course stationary. Whether every branch point of a stationary codimension 1 varifold must be a limit point of classical singularities remains an extremely interesting, highly nontrivial open problem. At the "opposite end" of this is the well-known open question of whether there exists a stationary codimension 1 varifold with an isolated singularity that is a branch point.

2.3. Multiplicity 1 classes: Simon's work. Let $B=B_{1}^{n+1}(0)$ be the open unit ball in $\mathbb{R}^{n+1}$ and let $\mathcal{V}_{1}$ be a family of $m$-varifolds $V$ on $B$ with $0 \in$ spt $\|V\|$ satisfying the following hypotheses:

(i) each $V \in \mathcal{V}_{1}$ is stationary in $B$;

(ii) if $V \in \mathcal{V}_{1}$ then $\Theta(\|V\|, Y)=1$ for $\|V\|$-a.e. $Y \in \operatorname{spt}\|V \mid\| \cap B$; 
(iii) if $V \in \mathcal{V}_{1}, Y \in \operatorname{spt}\|V\|, \rho \in(0$, dist $(Y, \partial B)]$ and $\Gamma$ is an orthogonal transformation of $\mathbb{R}^{n+1}$ then $\left(\Gamma_{\#} \eta_{Y, \rho \#} V\right)\left\llcorner B \in \mathcal{V}_{1}\right.$;

(iv) if $V_{j} \in \mathcal{V}_{1}$ for $j=1,2,3, \ldots, V$ is a varifold on $B$ and $V_{j} \rightarrow V$ as varifolds on $B$, then $V \in \mathcal{V}_{1}$.

In view of property (ii), such a class $\mathcal{V}_{1}$ is said to be a multiplicity 1 class. Examples of multiplicity 1 classes include the set of varifolds associated with codimension 1 locally area minimizing rectifiable currents $T$ on $B$ with $\partial T=0$ in $B$ and $0 \in$ spt $\|T\|$, and the set of multiplicity 1 varifolds associated with mod 2 minimizing currents $T$ on $B$ with $0 \in \operatorname{spt}\|T\|$.

By (iii) and (iv), every tangent cone $\mathbf{C}$ to a varifold $V \in \mathcal{V}_{1}$ belongs to $\mathcal{V}_{1}$ and hence inherits property (ii); in particular, branch points in $V \in \mathcal{V}_{1}$ are ruled out a priori. Consequently, by Allard's Regularity Theorem (Theorem 1.2),

$$
\operatorname{sing} V=\{x \in \operatorname{spt}\|V\|: \operatorname{dim} S(\mathbf{C}) \leq m-1 \quad \forall \mathbf{C} \in \operatorname{VarTan}(V, x)\} .
$$

Hence by the Almgren-Federer Generalized Stratification Theorem (Theorem 2.1), we see that if $V \in \mathcal{V}_{1}$, then there exists a non-negative integer $k_{V} \leq m-1$ such that $\operatorname{dim}_{\mathcal{H}}(\operatorname{sing} V) \leq k_{V}$. Let

$$
k_{\mathcal{V}_{1}}=\max _{V \in \mathcal{V}_{1}} k_{V}
$$

For example, for the class $\mathcal{M}_{1}$ of varifolds associated with $n$-dimensional locally area minimizing rectifiable codimension 1 currents, it follows from Theorem 2.1 and a theorem of J. Simons (Theorem 3.1 below) that this number $k_{\mathcal{M}_{1}}=n-7$.

In the seminal work [Sim83b], [Sim93], [Sim95], L. Simon developed a powerful set of a priori estimates applicable to any stationary varifold $V$ near a point at which $V$ has a multiplicity 1 tangent cone with an isolated singularity, and to any stationary varifold $V$ in a given multiplicity 1 class $\mathcal{V}_{1}$ near a point where $V$ has a tangent cone $\mathbf{C}$ with $\operatorname{dim} S(\mathbf{C})=k_{\mathcal{V}_{1}}$. Simon used these estimates to deduce for a stationary varifold uniqueness of any multiplicity 1 tangent cone with an isolated singularity, i.e. Theorem 2.2(a) below ([Sim83b]; see [Sim96] for a simplification of the argument), and a remarkably general structure theorem for singular sets of stationary varifolds belonging to a multiplicity 1 class, i.e. Theorem 2.2(b) below ([Sim95]).

Theorem 2.2 (Simon ([Sim83b], [Sim95])).

(a) If $V$ is a stationary integral m-varifold on $B, Z \in \operatorname{sing} V, \mathbf{C}$ is a multiplicity 1 tangent cone to $V$ at $Z$ with $\operatorname{sing} \mathbf{C}=\{0\}$, then $\mathbf{C}$ is the unique tangent cone to $V$ at $Z$.

(b) Let $\mathcal{V}_{1}$ be a multiplicity 1 class of stationary integral m-varifolds on $B$ and let $k=k_{\mathcal{V}_{1}}$ be as defined above. If $V \in \mathcal{V}_{1}$, then for any closed ball $D \subset B, \operatorname{sing} V \cap D$ consists of a finite number of pairwise disjoint locally $k$-rectifiable sets. 
Simon's work also provides an estimate giving the rate of asymptotic decay of $V$ upon rescaling to its unique tangent cone in case (a), and a similar estimate at $\mathcal{H}^{k}$-a.e. point along sing $V$ in case (b).

In Theorem 2.2(b), there is no hypothesis guaranteeing, near a given singularity at which there is a tangent cone $\mathbf{C}$ with $\operatorname{dim} S(\mathbf{C})=k_{\mathcal{V}_{1}}$, the existence of other singularities at all scales. Correspondingly, the conclusion of the theorem allows for "gaps" in the singular set at arbitrarily small scales. Whether such gaps ever exist in case $k_{\mathcal{V}_{1}} \geq 1$ remains an important open question however. Earlier work of Taylor ([Tay73], [Tay76]) and White ([Whi85]) established local $C^{1, \alpha}$ regularity for the singular sets in certain cases where there are topological obstructions for existence of such gaps in the singular sets.

Simon's work [Sim93] proves Theorem 2.2(b) for multiplicity 1 classes $\mathcal{V}_{1}$ whenever a certain additional integrability hypothesis on the crosssections of the cones $\mathbf{C}$ in $\mathcal{V}_{1}$ with $\operatorname{dim} S(\mathbf{C})=k_{\mathcal{V}_{1}}$ is satisfied, and establishes for $V$ belonging to such a class $\mathcal{V}_{1}$ a stronger result on asymptotic

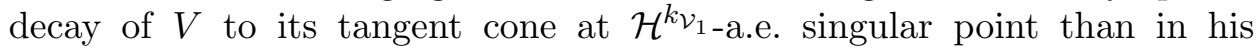
work [Sim95] (where the theorem is proved as stated above with no such integrability hypothesis). In case this integrability hypothesis is satisfied by C, Theorem 2.2(a) was known by earlier work of Allard-Almgren ([AA81]). (See [Sim85] for a concise presentation of the Allard-Almgren proof.)

As it turns out, appropriate versions of Simon's estimates in [Sim93] play a crucial role in the regularity theory, established recently in [Wic14a], for the class $\mathcal{S}$ of stable codimension 1 integral varifolds with no classical singularities, although $\mathcal{S}$ is not a multiplicity 1 class. (For $V \in \mathcal{S}$, the validity of the key requirement (ii) in the definition of multiplicity 1 class is a priori not clear at all-and is indeed literally not true - although a posteriori one has that $\Theta(\|V\|, X)$ is constant on connected components of spt $\|V\|$ away from a lower dimensional singular set and hence (ii) holds after normalization; the requirement (iv) fails for $\mathcal{S}$ as can easily be seen by considering, for instance, $V_{j} \in \mathcal{S}$ consisting of two multiplicity 1 parallel planes converging to a multiplicity 2 plane as $j \rightarrow \infty$.) We shall state the main content of the regularity theory for $\mathcal{S}$ in Section 3 below, and describe a sample of Simon's estimates and their significance for $\mathcal{S}$ in Part 2 where we describe the proof of the regularity results for $\mathcal{S}$ following [Wic14a]. Here we wish to make two points concerning these estimates in the context of the work [Wic14a]: (a) their role in [Wic14a] is indirect in the sense that they are established in contradiction arguments in order to rule out certain possibilities for $\mathcal{S}$ (specifically, to show that the class of functions obtained by "blowing up" sequences of varifolds in $\mathcal{S}$ converging to a hyperplane inherits the no-classical-singularities property from $\mathcal{S}$, and to show that the varifold closure of $\mathcal{S}$ does not contain singular cones supported on half-hyperplanes meeting along common axes); (b) since $\mathcal{S}$ is not a multiplicity 1 class, the hypotheses under which these estimates are established in [Wic14a] are 
different from those of $[\mathbf{S i m 9 3}]$, and consequently their proofs require a considerable amount of additional effort.

Besides the role played in [Wic14a], Simon's groundbreaking ideas in his work described above have had a profound impact on many other problems concerning singularities of solutions to geometric variational problems and geometric flows. Some of the most recent developments among the long list of theorems inspired by his work are: (i) the deep work of Colding-Minicozzi ([CM1 $],[\mathbf{C M 2}])$ and the work of Tonegawa and the author $([\mathbf{T W}])$ that establish certain parabolic versions of Simon's results to study asymptotics near certain singularities for mean curvature flow of hypersurfaces and for 1dimensional integral Brakke flows respectively, and (ii) the work of Krummel and the author $([\mathbf{K W} \mathbf{1}],[\mathbf{K W 2}],[\mathbf{K r u m 2}],[\mathbf{W i c}])$ on the structure of branch points of certain classes of minimal submanifolds and associated multiple valued harmonic functions, and the work of Hughes ([Hug]) on the structure of singularities of stationary two-valued Lipschitz graphs in arbitrary dimension and codimension.

\section{Stable varifolds}

We shall continue to denote by $U$ an open subset of $\mathbb{R}^{n+1}$. Let $V$ be a stationary integral $m$-varifold on $U$.

3.1. Second variation and stability. We say that $V$ is stable in $U$ if $V$ has non-negative second variation with respect to the area functional for all $C^{1}$ deformations of $U$ that leave a neighborhood of $\partial U \cup \operatorname{sing} V$ fixed and move reg $V$ in the normal direction. More precisely, $V$ is stable if

$$
\left.\frac{d^{2}}{d t^{2}}\right|_{t=0}\left\|\varphi_{\psi, t \#} V\right\|(U) \geq 0
$$

for any vector field $\psi \in C_{c}^{1}\left(U \backslash \operatorname{sing} V ; \mathbb{R}^{n+1}\right)$ with $\psi(X) \in\left(T_{X} \operatorname{reg} V\right)^{\perp}$ for each $X \in \operatorname{reg} V \cap \operatorname{spt} \psi$, where $\left(T_{X} \operatorname{reg} V\right)^{\perp}$ is the orthogonal complement of $T_{X} \operatorname{reg} V$ in $\mathbb{R}^{n+1}$ and $\varphi_{\psi, t}(X)=X+t \psi(X)$ for sufficiently small $t$.

3.2. Stability inequality in codimension 1. Let $V$ be stable, $m=n$ (i.e. $V$ be of codimension 1), and suppose that $\operatorname{reg} V$ is orientable with $\nu$ a continuous choice of unit normal vector field on $\operatorname{reg} V$. Then for any given $\zeta \in C_{c}^{\infty}(\operatorname{reg} V)$, we may take the vector field $\psi$ in (3.1) to be a suitable extension to $U$ of $\zeta \nu$. This leads to the following simple inequality ([[Sim83a], Sec. 9]), known as the codimension 1 stability inequality:

$$
\int_{\operatorname{reg} V}|A|^{2} \zeta^{2} d \mathcal{H}^{n} \leq \int_{\operatorname{reg} V}|\nabla \zeta|^{2} d \mathcal{H}^{n} \quad \forall \zeta \in C_{c}^{\infty}(\operatorname{reg} V) .
$$

Here $A$ denotes the second fundamental form of the embedding $\operatorname{reg} V \subset U$, $|A|$ the length of $A$ and $\nabla$ the gradient operator on $\operatorname{reg} V$. Note that (3.2) is equivalent to non-negativity of the Dirichlet eigenvalues of the self-adjoint 
linear differential operator $\mathcal{L}$ (known as the stability operator, or the Jacobi operator) given by $\mathcal{L} \zeta=\Delta \zeta+|A|^{2} \zeta$ for $\zeta \in C^{2}(\operatorname{reg} V)$.

Note also that (3.2) implies that $\int_{\text {reg } V \cap K}|A|^{2}<\infty$ for each compact set $K \subset U \backslash \operatorname{sing} V$. However, it is not at all clear whether it implies that $\operatorname{reg} V$ has locally finite total curvature in $U$ in the sense that

$$
\int_{\text {reg } V \cap K}|A|^{2}<\infty \quad \text { for each compact } K \subset U \text {. }
$$

A potential obstruction to such local finiteness of total curvature in stable codimension 1 integral varifolds is the presence of a "large" set of singularities that are not classical singularities. How large the set of non-classical singularities of a stable codimension 1 integral varifold $V$ is remains an open question except in cases when $V$ satisfies one of the following two additional conditions, each of which implies local finiteness of total curvature (in fact a uniform local $L^{2}$ bound on $\left.|A|\right)$ :

(i) all singularities of $V$ are non-classical;

(ii) $\Theta(\|V\|, X)<3$ and $\Theta(\|V\|, X) \notin\{3 / 2,5 / 2\}$ for each $X \in U$ (in particular, $\Theta(\|V\|, X)<3$ for each $X \in U$ and $V$ corresponds to a rectifiable current with no boundary in $U$ ).

In case (i), the singular set of $V$ is in fact known to have codimension 7 (by Theorem 3.3 below).

Local finiteness of total curvature in case (ii) is particularly interesting since in that case branch points can occur (unlike in case (i)), and when they do, for topological reasons they always arise along a set with locally positive $(n-2)$-dimensional Hausdorff measure. However the branch locus of $V$ in that case is locally the finite union of locally $(n-2)$-rectifiable sets (by [Wic] and [KW2]), and in particular has 2 capacity zero, and the non-classical, non-branch point singularities form a lower dimensional set.

3.3. Non-existence of singular stable hypercones in low dimensions: Simons' theorem. Any tangent cone to a stationary integral varifold at a non branch point singularity is necessarily singular at the origin. Moreover, if for a compact class $\mathcal{C}$ of $m$-dimensional stationary integral varifolds on the open unit ball $B=B_{1}^{n+1}(0)$ of $\mathbb{R}^{n+1}$ branch point singularities can be ruled out, then it follows from the Almgren-Federer Generalized Stratification (Theorem 2.1) that the singular set of a varifold in that class has Hausdorff dimension $\leq m-k$ where $k \in\{1,2, \ldots, m\}$ is the lowest integer such that there is a stationary cone $\mathbf{C}_{0} \in \operatorname{IV}_{k}\left(\mathbb{R}^{n+1-m+k}\right)$ with $\operatorname{sing} \mathbf{C}_{0}=\{0\}$ and $\mathbf{C}_{0} \times \mathbb{R}^{m-k}\left\llcorner B \in \mathcal{C}\right.$. (Here $\mathbf{C}_{0} \times \mathbb{R}^{m-k}$ has the obvious meaning: it is the varifold $\mathbf{C}$ with spt $\|\mathbf{C}\|=\operatorname{spt}\left\|\mathbf{C}_{0}\right\| \times \mathbb{R}^{m-k}$ and density given by $\Theta(\|\mathbf{C}\|,(x, y))=\Theta\left(\left\|\mathbf{C}_{0}\right\|, x\right)$ for $(x, y) \in \mathbb{R}^{n+1-m+k} \times \mathbb{R}^{m-k}$.) For this reason, the following result of J. Simons ([SJ68]) is of fundamental importance for the regularity theory of stable codimension 1 varifolds: 
Theorem 3.1 (Simons ([SJ68])). If $2 \leq k \leq 6$ and $\mathbf{C}_{0}$ is a $k$ dimensional stationary cone in $\mathbb{R}^{k+1}$ with sing $\mathbf{C}_{0} \subset\{0\}$ and $\mathbf{C}_{0}\left\llcorner\left(\mathbb{R}^{k+1} \backslash\right.\right.$ $\{0\})$ stable, then $\operatorname{sing} \mathbf{C}_{0}=\emptyset$ and hence spt $\left\|\mathbf{C}_{0}\right\|$ is a hyperplane in $\mathbb{R}^{k+1}$.

This theorem is obviously false in dimension $n=1$ ( consider a pair of lines in the plane crossing transversely). It is also false in dimensions $n \geq 7$. For instance the cone over $\frac{1}{\sqrt{2}} \mathbb{S}^{3} \times \frac{1}{\sqrt{2}} \mathbb{S}^{3} \subset \mathbb{R}^{8}$ can easily be seen to be stable (a fact observed first by Simons in [SJ68]), by taking in the first variation formula 1.3 the vector field $\psi(x)=|x|^{-2} \zeta(x) x$ for $\zeta \in C_{c}^{1}\left(\mathbb{R}^{8} \backslash\{0\}\right)$ and noting that norm of the second fundamental form of this cone is given by $|A(X)|=\sqrt{6}|X|^{-1}$.

\subsection{Area minimizing hypersurfaces: the work of De Giorgi, Federer-Fleming, Federer and Hardt-Simon.}

The combined work of De Giorgi ([DG61]), Federer-Fleming ([FF60]), Federer $([$ Fed70 $]$ ) together with Simons' theorem above (Theorem 3.1) lead to the fundamental interior regularity result given below in Theorem 3.2(a) for locally area minimizing hypersurfaces. This result was achieved over the decade 1960-70 with the work of Reifenberg ([Rei60]), Fleming ([Fle62]) and Almgren ([Alm66]) contributing at various stages. The subsequent work of Hardt-Simon ([HS79]) completed the theory for the Plateau problem by establishing complete boundary regularity (Theorem 3.2(b)) whenever the boundary is the multiplicity 1 current associated with an embedded $C^{1, \alpha}$ submanifold.

TheOREM 3.2 (Regularity of area minimizing hypersurfaces). Let $U$ be an open subset of $\mathbb{R}^{n+1}$.

(a) (De Giorgi [DG61], Federer-Fleming [FF60], Federer [Fed70], Simons [SJ68]) If $T$ is an $n$-dimensional, locally area minimizing rectifiable current in $U$ with $\partial T=0$ in $U$, then $\operatorname{sing} T=\emptyset$ in case $1 \leq n \leq 6, \operatorname{sing} T$ is discrete in case $n=7$ and $\operatorname{dim}_{\mathcal{H}}(\operatorname{sing} T) \leq$ $n-7$ in case $n \geq 8$.

(b) (Hardt-Simon [HS79]) If $T$ is an $n$-dimensional rectifiable current in $U$ such that $T$ is locally area minimizing in $U$ away from $\partial T$, and $\partial T$ is equal to the multiplicity 1 current associated with a connected, oriented embedded $C^{1, \alpha}$ submanifolds of $U$ for some $\alpha \in(0,1)$, then there is an open subset $V$ containing spt $\partial T$ such that $V \cap \operatorname{spt} T$ is a connected embedded $C^{1, \alpha}$ hypersurface-withboundary.

3.5. The Bernstein Theorem. Codimension 1 minimal graphs are a special class of oriented locally area minimizing (in fact calibrated) hypersurfaces. These are hypersurfaces $G$ that are graphs over a domain $\Omega \subset \mathbb{R}^{n}$ 
of functions $u: \Omega \rightarrow \mathbb{R}$ solving the minimal surface equation

$$
\sum_{j=1}^{n} D_{j}\left(\frac{D_{j} u}{\sqrt{1+|D u|^{2}}}\right)=0 \text { in } \Omega .
$$

The well-known Bernstein theorem asserts that if $G$ is entire, that is if $\Omega=\mathbb{R}^{n}$, then $u$ is an affine function in case $n \leq 7$; this is a triviality in case $n=1$ and was first proved by Bernstein for $n=2$ ([Ber15]). Using an observation due to Fleming ([Fle62] $)$ that showed that it suffices to show that one tangent cone to the graph at infinity is a (multiplicity 1 ) hyperplane, De Giorgi extended the theorem to the case $n=3$ ([DG65]) and Almgren to the case $n=4$ ([Alm65]). De Giorgi in fact showed that in any dimension, a tangent cone to the minimal graph at infinity splits a direction. Subsequently, Simons' classification theorem above (Theorem 3.1) extended the Bernstein Theorem to dimensions $n \leq 7$. Bombieri-De Giorgi-Giusti ([BDG69]) showed, remarkably, that the theorem is false in dimensions $n \geq 8$, by explicitly exhibiting a construction producing a non-planar entire minimal graph $G_{n}$ asymptotic at infinity to the "vertical cylindrical cone" $\mathbf{C}_{n-1} \times \mathbb{R}$ in every even dimension $n \geq 8$ (which also of course gives such a graph in every odd dimension $n \geq 9$ by taking, for instance, the product $\left.G_{n-1} \times \mathbb{R}\right)$, where $\mathbf{C}_{n}$ is the cone over $\frac{1}{\sqrt{2}} \mathbb{S}^{\frac{n-1}{2}} \times \frac{1}{\sqrt{2}} \mathbb{S}^{\frac{n-1}{2}} \subset \mathbb{R}^{n+1}$.

3.6. Curvature estimates under a priori regularity hypotheses: the work of Schoen, Schoen-Simon-Yau and Schoen-Simon.

Various local estimates have been established for low dimensional smooth stable minimal hypersurfaces, which can be regarded as the local versions of the Bernstein type global theorems. The first such result is due to Heinz ([Hei52]) who established a local curvature estimate for two dimensional minimal graphs, i.e. for $G=\operatorname{graph} u$ with $u$ a solution to the minimal surface equation in a domain $U \subset \mathbb{R}^{2}$; Heinz's estimate says that there exists a fixed constant $C$ independent of $G$ such that the second fundamental form $A_{G}$ of $G$ satisfies

$$
\left|A_{G}\right|(x, u(x)) \leq C R^{-1}
$$

whenever $B_{R}(x) \subset U$. The (two dimensional) Bernstein Theorem follows immediately from this since one can simply let $R \rightarrow \infty$ in the estimate in case $U=\mathbb{R}^{2}$.

Schoen ([Scho83]) generalized Heinz's result to the parametric setting by proving the beautiful result that there is a fixed constant $C$ such that

$$
\left|A_{M}\right|(X) \leq C(\operatorname{dist}(X, \partial M))^{-1}
$$

for every oriented properly immersed stable minimal surface $M$ in $\mathbb{R}^{3}$ and $X \in M \backslash \partial M$, where $\operatorname{dist}(X, \partial M)$ is the geodesic distance from $X$ to the boundary $\partial M$. This in particular implies the result that a complete oriented immersed stable minimal surface without boundary in $\mathbb{R}^{3}$ must be a plane. This last result had been established earlier independently by 
do Carmo and Peng ([DP79]), by Fischer-Colbrie and Schoen ([FS80]) and by Pogorelev ([Pog81] $)$, and is an extension to the parametric setting of the two dimensional graphical Bernstein Theorem stated above. The Catenoid shows that without the stability assumption such a global (and hence also a local) result is false even if the surface has finite total curvature. It remains a very interesting open question whether in any of the dimensions $n \in\{3,4,5,6\}$, Schoen's local curvature estimate, or its global version asserting non-existence of non-trivial complete stable minimal hypersurfaces in $\mathbb{R}^{n+1}$, holds true. Such a result cannot hold if $n \geq 7$ (see below).

Allowing the constant $C$ to depend on the mass ratio with respect to geodesic balls, the above curvature estimate was extended to properly immersed stable hypersurfaces of dimension $n \leq 5$ by Schoen-Simon-Yau in their highly influential work [SSY75]. The Schoen-Simon-Yau estimate asserts that there exists a fixed constant $C$ (independent of mass ratio) such that if $n \leq 5$ and if $M$ is an oriented immersed stable minimal hypersurface of $\mathbb{R}^{n+1}$, then for each $X \in M \backslash \partial M$ and each $R \in(0$, dist $(X, \partial M)$ ) (where again dist $(X, \partial M)$ is the geodesic distance from $X$ to $\partial M)$,

$$
\left|A_{M}(X)\right| \leq C R^{-n-1} \mathcal{H}^{n}\left(D_{R}^{M}(X)\right)
$$

where $D_{R}^{M}(X)$ is the geodesic ball in $M$ of radius $R$ and center $X$.

It remains open whether the Schoen-Simon-Yau estimate holds for uniformly mass bounded properly immersed stable hypersurfaces of dimension $n=6$. In the special case when $M$ is a 6 dimensional immersed stable hypersurface satisfying the particular mass bound $\mathcal{H}^{6}\left(M \cap B_{R}^{7}(X)\right) \leq(3-\delta) \omega_{6} R^{6}$ for some $\delta \in(0,1)$, the estimate (in the form $\left|A_{M}(X)\right| \leq C R^{-1}$ with $C=C(\delta))$ is known to be true ([Wic08]).

A local curvature estimate of the above type cannot hold for area minimizing hypersurfaces of dimension $n \geq 7$ since in these dimensions there are non-planar complete locally area minimizing hypersurfaces; for instance, it is known by the work of Hardt-Simon ([HS85]) that each of the connected components of $\mathbb{R}^{n+1} \backslash \mathbf{C}_{n}$, where $\mathbf{C}_{n}$ is the cone over the product of spheres mentioned above, can be foliated by smooth locally area minimizing hypersurfaces. For $n \geq 8$, we also have the Bombieri-De GiorgiGiusti ([BDG69]) examples of non-planar entire minimal graphs over $\mathbb{R}^{n}$ mentioned above which are automatically locally area minimizing. These graphical examples show that Heinz' local curvature estimate stated above does not hold for minimal graphs over domains in $\mathbb{R}^{n}$ for $n \geq 8$; it does however hold if $n \leq 7$.

In their fundamental work [SS81], Schoen and Simon showed that in arbitrary dimension $n$, the preceding curvature estimate in the form $\left|A_{M}(X)\right| \leq C R^{-1}$ with $C=C(n, \Lambda)$ where $\Lambda$ is any upper bound for $R^{-n} \mathcal{H}^{n}\left(M \cap B_{R}^{n+1}(X)\right)$ holds for embedded $n$-dimensional stable minimal hypersurfaces $M$ provided that $M$ is sufficiently close (depending only on $n$ and $\Lambda$ ) in Hausdorff distance to a hyperplane. In fact they proved this result allowing a small singular set (see Theorem 6.2 below and Remark 2 following 
it). From this result they deduced the curvature estimate $\left|A_{M}(X)\right| \leq$ $C R^{-1}, C=C(\Lambda)$, for 6-dimensional oriented properly embedded stable hypersurfaces $M$ satisfying the volume growth bound $R^{-6} \mathcal{H}^{6}\left(M \cap B_{R}^{7}(X)\right) \leq$ $\Lambda$.

\subsection{A general theory: stable codimension 1 varifolds with no classical singularities.}

The following recent theorem, which shall be the main focus of the rest of the paper, says that a stable codimension 1 integral varifold with no classical singularities has no branch points, and moreover, is smoothly embedded away from a closed singular set of codimension $\geq 7$. In particular the theorem implies that such a hypersurface is free of singularities if $n \leq 6$.

THEOREM 3.3 (Regularity and compactness for stable codimension 1 integral varifolds; [[Wic14a], Theorem 3.1]). Let $U$ be an open subset of $\mathbb{R}^{n+1}, V \in \mathrm{IV}_{n}(U)$ and suppose that $V$ has locally finite mass in $U$ and that

(i) $V$ is stationary in $U$;

(ii) $V\llcorner B$ is stable in $B$ for each open ball $B \subset U$ with $\operatorname{sing} V\llcorner B=\emptyset$ in case $1 \leq n \leq 6, \operatorname{sing} V\llcorner B$ discrete in case $n=7$, or

$$
\operatorname{dim}_{\mathcal{H}}(\operatorname{sing} V\llcorner B) \leq n-7
$$

in case $n \geq 8$.

If $V$ has no classical singularities, then $V$ has no branch points (see definitions in Section 2.2); in this case, $\operatorname{sing} V=\emptyset$ if $1 \leq n \leq 6, \operatorname{sing} V$ is discreet if $n=7$ and $\operatorname{dim}_{\mathcal{H}} \operatorname{sing} V \leq n-7$ if $n \geq 8$. Furthermore, for any $\Lambda>0$, the set

$$
\begin{aligned}
& \left\{V \in \operatorname{IV}_{n}(U):\right. \\
& \quad\|V\|(U) \leq \Lambda, V \text { satisfies (i), (ii) and has no classical singularities }\}
\end{aligned}
$$

is compact in the topology of varifold convergence in $U$.

This result essentially completes the codimension 1 theory for stable varifolds in the "embedded case" (which for varifolds it makes sense to take as the case in which classical singularities are absent). It generalizes both the classical theory for oriented area minimizing hypersurfaces (Theorem 3.2(a)) and the Schoen-Simon theory (described briefly in the last paragraph of Section 3.6 above and in more detail in Section 6.1 below). Theorem 3.3 is sharp with respect to all of its hypotheses and conclusions in ways that have been discussed in the introduction.

We shall describe some applications of Theorem 3.3 in the next section, and give in Part 2 below a description of the strategy and the main ingredients of its proof. 


\section{Some applications of the general theory}

In this section we discuss three applications of Theorem 3.3. The first two applications concern, respectively, the strong maximum principle and the unique continuation property for stationary codimension 1 integral varifolds, as established in [Wic14b]. They only require the special case of Theorem 3.3 with hypothesis $\mathcal{H}^{n-1}(\operatorname{sing} V)=0$ in place of the noclassical-singularities hypothesis. The third application discussed below is to a class of semi-linear elliptic problems. It concerns regularity of the sharp interface resulting in the limit as the perturbation parameter tends to zero of a sequence of stable critical points of the Allen-Cahn functionals, as established in the work of Tonegawa and the author ([TW10]). This result requires the full strength of Theorem 3.3.

4.1. Strong maximum principle for stationary codimension 1 varifolds. Let $U$ be an open subset of $\mathbb{R}^{n+1}$, and let $M_{1}, M_{2}$ be two connected, smooth minimal hypersurfaces of $U$ with no singularities in $U$ (thus $\left(\operatorname{clos} M_{j} \backslash M_{j}\right) \cap U=\emptyset$ for $j=1,2$ ). Suppose, locally near every common point of the hypersurfaces, one of them lies on one side of the other. That is to say, for each $y \in M_{1} \cap M_{2}$ there is $\rho>0$ such that $B_{\rho}(y) \backslash M_{2}$ consists precisely of two connected components (as it must for sufficiently small $\rho>0)$ and $M_{1} \cap B_{\rho}(y)$ is contained in the closure of one of the components of $B_{\rho}(y) \backslash M_{2}$. (Interchanging $M_{1}$ and $M_{2}$ in this statement of course gives an equivalent condition.) Then it is an easy consequence of the strong maximum principle for uniformly elliptic PDE that either $M_{1}=M_{2}$ or $M_{1} \cap M_{2}=\emptyset$.

It is a natural question to ask to what extent this strong maximum principle extends to pairs of stationary codimension 1 integral varifolds $V_{1}$, $V_{2}$ on $U$ with connected supports. This question is subtle since we do not know much about the singular sets of stationary varifolds. Note that to make the question precise, one has to define what it means for one varifold to lie locally on one side of the other near a common point, and how to even do this is not entirely obvious for it is not known in the presence of singularities whether the support of a stationary codimension 1 integral varifold must disconnect a sufficiently small ambient ball around a singularity; moreover, even when it does, it obviously need not be into two components (take for instance a pair of crossed planes in $\mathbb{R}^{3}$ ). A natural condition to assume is that whenever the support of one varifold does disconnect a ball around a common point into two components, the part of the support of the other varifold in that ball is contained in the closure of one of the two components. As it turns out, the weaker condition given below suffices for a sharp theorem:

Hypothesis $K\left(V_{1}, V_{2}\right)$ : If $y \in \operatorname{reg} V_{1} \cap \operatorname{spt}\left\|V_{2}\right\|$ and $\rho>0$ are such that (i) $\operatorname{sing} V_{1} \cap B_{\rho}(y)=\emptyset$ and (ii) $B_{\rho}(y) \backslash$ spt $\left\|V_{1}\right\|$ consists of two connected components (both of which hold for every $y \in \operatorname{reg} V_{1} \cap \operatorname{spt}\left\|V_{2}\right\|$ 
and sufficiently small $\rho>0)$, then spt $\left\|V_{2}\right\| \cap B_{\rho}(y)$ is contained in the closure of one of the components of $B_{\rho}(y) \backslash \operatorname{spt}\left\|V_{1}\right\|$.

(Spoiler: our choice of terminology here is based on the fact that the letter $K$ consists of a regular piece and a singular piece with the singular piece entirely on one side of the regular piece!)

Easy examples (see Remark 1 below) show that two stationary codimension 1 integral varifolds $V_{1}, V_{2}$ satisfying Hypothesis $K\left(V_{1}, V_{2}\right)$ may have connected, intersecting, distinct supports, and hence need not satisfy the strong maximum principle. However, this cannot happen if the singular set of $V_{1}$ is sufficiently small. The following result, which builds upon and sharpens previous maximum principles due to Moschen [Mos77], Simon [Sim87], Solomon-White [SW89] and Ilmanen [Ilm96], gives the sharp size hypothesis on $\operatorname{sing} V_{1}$ for the maximum principle to hold whenever $K\left(V_{1}, V_{2}\right)$ holds, and is obtained as a consequence of Theorem 3.3. (See Section 4.3 for some comments on its proof.)

TheOREM 4.1 (Strong maximum principle; [Wic14b]). Let $V_{1}, V_{2}$ be stationary codimension 1 integral $n$-varifolds on a smooth Riemannian manifold such that spt $\left\|V_{j}\right\|$ is connected for $j=1,2$. If

(i) spt $\left\|V_{2}\right\|$ lies locally on one side of $\operatorname{reg} V_{1}$ in the sense that Hypothesis $K\left(V_{1}, V_{2}\right)$ above holds and

(ii) $\mathcal{H}^{n-1}\left(\operatorname{sing} V_{1}\right)=0$, then either spt $\left\|V_{1}\right\| \cap \operatorname{spt}\left\|V_{2}\right\|=\emptyset$ or $\operatorname{spt}\left\|V_{1}\right\|=\operatorname{spt}\left\|V_{2}\right\|$.

Remarks: (1) The hypothesis (ii) cannot be weakened to $\mathcal{H}^{n-1+\epsilon}\left(\operatorname{sing} V_{1}\right)$ $=0$ for any $\epsilon>0$; to see this, consider for instance four intersecting planes in $\mathbb{R}^{3}$ with a common axis and with at least three of the planes distinct, and let $V_{1}$ be the union of an "inner" pair of planes and $V_{2}$ be the union of the corresponding "outer" pair (each with multiplicity 1). A similar counterexample in which neither varifold is the sum of two non-trivial stationary varifolds and their regular parts have non-empty intersection is obtained by taking $V_{1}, V_{2}$ to be the multiplicity 1 varifolds supported on three and, respectively five, equally spaced half-planes meeting along a common axis.

(2) The theorem is also sharp with respect to hypothesis (i) in the sense that it is not enough to require merely that reg $V_{2}$ lies locally on one side of reg $V_{1}$ (that is, to verify Hypothesis $\mathrm{K}\left(V_{1}, V_{2}\right)$ merely for points $\left.y \in \operatorname{reg} V_{1} \cap \operatorname{reg} V_{2}\right)$; to see this, let $V_{2}$ be the multiplicity 1 varifold supported on the union of three equally spaced half-planes in $\mathbb{R}^{3}$ meeting along a common axis, and $V_{1}$ be the multiplicity 1 varifold supported on the plane containing one of the three half-planes of $V_{2}$.

(3) If $\Omega_{1}, \Omega_{2}$ are open subsets of the ambient manifold with $\Omega_{1} \subset \Omega_{2}$ and $V_{j}=\left|\partial \llbracket \Omega_{j} \rrbracket\right|$ for $j=1,2$ (i.e. $V_{j}$ is the $n$-varifold corresponding to the multiplicity 1 boundary of the $(n+1)$-dimensional current defined by the open set $\Omega_{j}$ ), then spt $\left\|V_{2}\right\|$ lies locally on one side of $\operatorname{reg} V_{1}$, and also spt $\left\|V_{1}\right\|$ lies locally on one side of reg $V_{2}$, in the sense that both Hypothesis 
$\mathrm{K}\left(V_{1}, V_{2}\right)$ and Hypothesis $\mathrm{K}\left(V_{2}, V_{1}\right)$ hold. So in this case, if $V_{1}, V_{2}$ are stationary; spt $\left\|V_{j}\right\|$ is connected for $j=1,2$; spt $\left\|V_{1}\right\| \cap$ spt $\left\|V_{2}\right\| \neq \emptyset$ and either $\mathcal{H}^{n-1}\left(\operatorname{sing} V_{1}\right)=0$ or $\mathcal{H}^{n-1}\left(\operatorname{sing} V_{2}\right)=0$, then it follows (from the theorem) that spt $\left\|V_{1}\right\|=\operatorname{spt}\left\|V_{2}\right\|$.

(4) Allowed in Hypothesis $\mathrm{K}\left(V_{1}, V_{2}\right)$ is the possibility that reg $V_{1} \cap \operatorname{spt}\left\|V_{2}\right\|=$ $\emptyset$, in which case, subject also to all other hypotheses of the theorem, the conclusion is that spt $\left\|V_{1}\right\| \cap \operatorname{spt}\left\|V_{2}\right\|=\emptyset$.

\subsection{Unique continuation for stationary codimension 1}

varifolds. The classical weak unique continuation property for solutions to the minimal surface system implies that if $M_{1}, M_{2}$ are $n$-dimensional connected, smoothly embedded minimal submanifolds of a Riemannian manifold with $\operatorname{sing} M_{j} \equiv \operatorname{clos} M_{j} \backslash M_{j}=\emptyset$ for $j=1,2$, and if $M_{1}, M_{2}$ intersect on a set of positive $(n-1+\gamma)$-dimensional Hausdorff measure for some $\gamma \in(0,1]$, then $M_{1}=M_{2}$. For stationary integral varifolds (with connected supports) this statement generally is false, even when the codimension is 1 , as can be seen by the simple example given in the remark below. However, we have the following result:

Theorem 4.2 (Unique continuation; [Wic14b]). Let $V_{1}, V_{2}$ be stationary codimension 1 integral n-varifolds on a Riemannian manifold such that spt $\left\|V_{j}\right\|$ is connected for $j=1,2$ and

$$
\mathcal{H}^{n-1+\gamma}\left(\operatorname{spt}\left\|V_{1}\right\| \cap \operatorname{spt}\left\|V_{2}\right\|\right)>0
$$

for some $\gamma \in(0,1]$. If $\mathcal{H}^{n-1}\left(\operatorname{sing} V_{j}\right)=0$ for $j=1,2$, then

$$
\text { spt }\left\|V_{1}\right\|=\operatorname{spt}\left\|V_{2}\right\| \text {. }
$$

Remark: The singular set hypothesis $\mathcal{H}^{n-1}\left(\operatorname{sing} V_{j}\right)=0, j=1,2$, in this theorem is sharp. To see that it is so, take for instance $V_{1}$ to be the multiplicity 1 varifold supported on a union of three half-planes in $\mathbb{R}^{3}$ meeting at $120^{\circ}$ angles along a common axis, and $V_{2}$ to be the multiplicity 1 varifold supported on the plane containing one of the three half-planes of spt $\left\|V_{1}\right\|$. It is also clear that the theorem does not hold with $\gamma=0$.

4.3. Remarks on the proofs of the maximum principle and the unique continuation theorem. The principal ingredient of the proofs of both Theorem 4.1 and Theorem 4.2 is the following result, which may also be of independent interest:

Theorem 4.3 ([Wic14b]). Let $V_{1}, V_{2}$ be stationary codimension 1 integral $n$-varifolds on a smooth Riemannian manifold. If

$$
\mathcal{H}^{n-1}\left(\operatorname{spt}\left\|V_{1}\right\| \cap \operatorname{spt}\left\|V_{2}\right\|\right)=0
$$

then $\mathrm{spt}\left\|V_{1}\right\|$ and $\mathrm{spt}\left\|V_{2}\right\|$ are disjoint.

Remark: A pair of transversely intersecting hyperplanes in a Euclidean space shows that the hypothesis $\mathcal{H}^{n-1}$ ( $\left.\operatorname{spt}\left\|V_{1}\right\| \cap \operatorname{spt}\left\|V_{2}\right\|\right)=0$ in Theorem 4.3 is sharp. 
In addition to Theorem 4.3, the proof of Theorem 4.1 also makes direct use of the special case of Theorem 4.1 when $\operatorname{sing} V_{1}=\emptyset$, which follows from [[Whi10], Theorem 4] (which in turn is a consequence of the main theorem in Solomon-White ([SW 89]) and Ilmanen's Lemma 10 in [Ilm96]). Given these ingredients, the proofs of Theorem 4.1 and Theorem 4.2 are fairly elementary, requiring only some basic facts about stationary varifolds, and the strong maximum principle and the unique continuation property for (smooth) solutions to the minimal surface equation. (See [Wic14b] for details.)

The proof of Theorem 4.3 follows from a clever argument of Ilmanen ([Ilm96]), and uses Theorem 3.3 in an essential way. Ilmanen ([Ilm96]) proved Theorem 4.3 subject to the stronger hypothesis that

$$
\mathcal{H}^{n-2}\left(\operatorname{spt}\left\|V_{1}\right\| \cap \operatorname{spt}\left\|V_{2}\right\| \cap K\right)<\infty
$$

for every compact subset $K$ of the ambient manifold (and used it to deduce the special case of Theorem 4.1 when both varifolds have singular sets of locally finite $(n-2)$-dimensional Hausdorff measure). Ilmanen's proof builds on results and ideas from the work of Solomon-White [SW89] and Simon [Sim87], and makes clever use of the Schoen-Simon version ([SS81]) of Theorem 3.3 (which requires, as mentioned before, that the singular sets have locally finite $(n-2)$-dimensional Hausdorff measure).

The first step of Ilmanen's argument in [IIm96] is to prove Theorem 4.3 assuming that $V_{1}, V_{2}$ are stable with singular sets of locally finite $(n-$ 2)-dimensional Hausdorff measure. This step uses a certain Jacobi field argument from [Sim87] and the results of [SS81].

The second step is to interpose two distinct stable hypersurfaces $W_{1}$, $W_{2}$ "between" the stationary varifolds $V_{1}, V_{2}$ to obtain a contradiction to the first step in case spt $\left\|V_{1}\right\|$, spt $\left\|V_{2}\right\|$ have non-empty intersection satisfying the above size restriction. The construction of $W_{1}, W_{2}$ in [Ilm96] is carried out by solving a certain obstacle problem in the complement of spt $\left\|V_{1}\right\| \cap$ apt $\|V\|_{2}$. This procedure yields by construction that for $j=1,2, \operatorname{sing} W_{j} \backslash \operatorname{spt}\left\|V_{1}\right\| \cap \operatorname{spt}\left\|V_{2}\right\|$ is empty if $n \leq 6$ and has hausdorff dimension $\leq n-7$ if $n \geq 7$, and that $W_{j}$ has Euclidean volume growth; moreover, one has that spt $\left\|V_{1}\right\| \cap \operatorname{spt}\left\|V_{2}\right\| \subset \operatorname{sing} W_{1} \cap \operatorname{sing} W_{2}$. To check stationarity of $W_{j}$ across spt $\left\|V_{1}\right\| \cap \mathrm{spt}\left\|V_{2}\right\|$, it therefore suffices to have $\mathcal{H}^{n-1}$ (spt $\left.\left\|V_{1}\right\| \cap \operatorname{spt} V_{2} \|\right)=0$, but to ensure that $\operatorname{sing} W_{j}$ has locally finite $(n-2)$-dimensional Hausdorff measure in order to appeal to the result of the first step, the above size restriction on spt $\left\|V_{1}\right\| \cap \operatorname{spt}\left\|V_{2}\right\|$ was needed.

Theorem 4.3 in the sharp form stated above follows from Ilmanen's argument, by using Theorem 3.3 at those places in the first step of the argument where it depended on the results of [SS81]. Once the first step is strengthened this way, the second step carries over with the assumption that $\mathcal{H}^{n-1}$ (spt $\left.\left\|V_{1}\right\| \cap \mathrm{spt}\left\|V_{2}\right\|\right)=0$ to produce $W_{1}, W_{2}$ contradicting the first step unless spt $\left\|V_{1}\right\| \cap \operatorname{spt}\left\|V_{2}\right\|=\emptyset$. 
4.4. Interface regularity for a class of phase transition problems. For $\epsilon \in(0,1)$, consider the family of functionals $E_{\epsilon}$ given by

$$
E_{\epsilon}(u)=\int_{\Omega} \frac{\epsilon|D u|^{2}}{2}+\frac{W(u)}{\epsilon} d x
$$

for $u \in H^{1}(\Omega)$, where $\Omega \subset \mathbb{R}^{n+1}$ is a bounded domain and

$$
W: \mathbb{R} \rightarrow \mathbb{R}^{+} \cup\{0\}
$$

is a "standard" $C^{3}$ double-well potential with non-degenerate minima precisely at \pm 1 with $W( \pm 1)=0$ (e.g. $\left.W(t)=\left(1-t^{2}\right)^{2}\right)$. Suppose

(i) $\epsilon_{1}, \epsilon_{2}, \epsilon_{3}, \ldots$ are positive numbers with $\lim _{j \rightarrow \infty} \epsilon_{j}=0$.

(ii) $u_{\epsilon_{j}} \in H^{1}(\Omega)$ and $E_{\epsilon_{j}}\left(u_{\epsilon_{j}}\right)+\sup _{\Omega}\left|u_{\epsilon_{j}}\right| \leq c$ for a fixed constant $c \geq 1$ and each $j=1,2,3 \ldots$

(iii) $u_{\epsilon_{j}}$ is a stable critical point of $E_{\epsilon_{j}}$ for each $j=1,2,3 \ldots$ Thus $-\epsilon_{i} \Delta u_{\epsilon_{j}}+\epsilon_{j}^{-1} W^{\prime}\left(u_{\epsilon_{j}}\right)=0$ weakly on $\Omega$ and $u_{\epsilon_{j}}$ satisfies

$$
\int_{\Omega} \epsilon_{j}|\nabla \phi|^{2}+\frac{W^{\prime \prime}\left(u_{\epsilon_{i}}\right)}{\epsilon_{j}} \phi^{2} \geq 0 \quad \text { for each } \phi \in C_{c}^{1}(\Omega),
$$

conditions that are equivalent, respectively, to

$$
\begin{gathered}
\left.\frac{d}{d t}\right|_{t=0} E_{\epsilon_{j}}\left(u_{\epsilon_{j}}+t \phi\right)=0 \quad \text { and } \\
\left.\frac{d^{2}}{d t^{2}}\right|_{t=0} E_{\epsilon_{j}}\left(u_{\epsilon_{j}}+t \phi\right) \geq 0 \quad \forall \phi \in C_{c}^{1}(\Omega) .
\end{gathered}
$$

Theorem 4.4 (Tonegawa-Wickramasekera; [TW10]). If (i), (ii), (iii) hold, then either

(a) $u_{\epsilon_{j}} \rightarrow 1$ or $u_{\epsilon_{j}} \rightarrow-1$ locally uniformly in $\Omega$, or

(b) after passing to a subsequence of $\left\{\epsilon_{j}\right\}$ without changing notation, for each fixed $s \in(0,1)$, the interface regions $\left\{x \in \Omega:\left|u_{\epsilon_{j}}(x)\right|<s\right\}$ converge locally in Hausdorff distance to a "sharp interface" $M$ where $M$ is a smooth, embedded stable minimal hypersurface of $\Omega$ with no removable singularities and $\operatorname{sing} M \equiv(\operatorname{clos} M \backslash M) \cap \Omega=\emptyset$ if $2 \leq n \leq 6, \operatorname{sing} M$ (at most) a discrete set if $n=7$ and $\operatorname{dim}_{\mathcal{H}} \operatorname{sing} M \leq n-7$ if $n \geq 8$.

This extends earlier work in the energy minimizing setting (studying the $\Gamma$-limits of sequences $E_{\epsilon_{j}}$ for $\epsilon_{j} \rightarrow 0^{+}$, see e.g. [MM77], [Mod87], [Ste88], [KS89]) in which case the limit-interface is locally area-minimizing. Combined work of Hutchinson-Tonegawa [HT00] and Tonegawa [Ton05] had previously established that in the stable case, the limit interface is an $n$-dimensional stable integral varifold $V$ on $\Omega$ but gave little information on the regularity of $V$ except when $n=1$, in which case it was shown in 
[Ton05] that $\operatorname{spt} V$ locally consists of finitely many disjoint straight line segments.

In the proof of Theorem 4.4 above, it is shown first that at each point in the complement of a certain set $Z \subset \operatorname{spt} V$ with $\mathcal{H}^{n-1}(Z)=0$ (but $Z$ not known to be closed a priori), no tangent cone to $V$ can be supported on the union of 3 or more half-hyperplanes meeting along a common axis. This suffices to apply very directly Theorem 3.3 above to $V$ because by the definition of classical singularity, if $V$ has such a singularity then the set of points where $V$ has a tangent cone supported on a union of 3 or more half-hyperplanes having a common axis must have positive $\mathcal{H}^{n-1}$ measure.

Note that the full strength of Theorem 3.3 is used here. In particular, because of the presence of the set $Z$ about which we know nothing a priori apart from the fact that $\mathcal{H}^{n-1}(Z)=0$, it would not suffice for the proof of Theorem 4.4 to have a version of Theorem 3.3 where in place of the no-classical-singularities hypothesis, one assumes either the condition $\mathcal{H}^{n-1}(\sin g)=0$, or the "asymptotic condition" that $V$ has no tangent cone supported on a union of 3 or more half-hyperplanes meeting long a common axis.

\section{Some questions arising from the recent work}

(1) In view of the work [TW10], it is natural to ask if the Almgren-PittsSchoen-Simon ([Alm65], [Pit77], [SS81]) existence theorem for embedded minimal hypersurfaces in compact Riemannian manifolds can be obtained via an approach based on the Cahn-Hilliard functional. Such an approach raises the possibility of replacing Pitts' construction of almost minimizing varifolds ([Pit77]; see also the work of De Lellis-Tasnady $[\mathbf{D T}]$ for a streamlined version of Pitts' argument) with a construction of suitably controlled critical points of the Cahn-Hilliard functionals with small perturbation parameter, i.e. a construction in a function space rather than in a varifold space. Further encouragement for this approach is provided by a recent argument of Sharp ([Sha $]$ ) which, combined with the main regularity theorem of [TW10] (Theorem 4.4 above), one expects would make it possible to relax the stability hypothesis in Theorem 4.4 to a uniform bound on the index for the sequence of critical points. An approach to the existence question along these lines may also give additional information such as a bound on the index of the minimal hypersurface obtained as the limit interface. Such bounds are not known for the Almgren-Pitts-Schoen-Simon solutions in general. (See however the work of Marques-Neves [MN12], [MN14] that establishes index bounds in certain special cases.)

(2) Do the strong maximum principle and the unique continuation theorem of [Wic14b] (Theorem 4.1 and Theorem 4.2 above) extend to stationary codimension 1 integral varifolds admitting no tangent cones supported on three or more half-hyperplanes meeting along an axis? Note that the easy examples given above illustrating sharpness of the size hypothesis on the 
singular sets in these theorems all have singularities of this type. A more ambitious question is whether these results extend to stationary codimension 1 integral varifolds with no classical singularities. Even more ambitiously, what can one say about the singular set of a stationary codimension 1 integral varifold with no classical singularities?

(3) In view of the standard terminology used in the corresponding PDE results, the unique continuation result for stationary varifolds discussed above (Theorem 4.2) might be termed a weak unique continuation theorem. On the PDE side, it is well known ([GL86], [GL87]) that solutions to second order homogeneous linear elliptic equations with Lipschitz leading order coefficients and bounded lower order coefficients satisfy a strong unique continuation property, in the sense that if the $L^{2}$ average of the solution in small balls centered at a fixed point decays arbitrarily fast with decreasing scale, then the solution is identically zero near that point. So a natural question is whether a strong unique continuation property holds for stationary varifolds. A special case of this question would be to ask if a stationary integral $n$-varifold (say of codimension 1 ) near some point in its support has height excess relative to an $n$-dimensional plane passing through that point decaying arbitrarily fast upon rescaling, whether the varifold must coincide with the plane in a neighborhood of that point. More generally, one could ask the same question with the plane replaced by another stationary integral $n$-varifold.

(4) Must the regular part of a stationary integral $n$-varifold in an open ball in $\mathbb{R}^{n+1}$ be orientable? Must the support of such a varifold disconnect a sufficiently small open ball about a singularity?

(5) As mentioned in the introduction, in [CM14] Colding and Minicozzi use Theorem 3.3 above to deduce that for $2 \leq n \leq 6$, an $n$-dimensional self-shrinker in $\mathbb{R}^{n+1}$ with polynomial volume growth, $F$-stable regular part and no classical singularities is either a round sphere or a hyperplane (possibly with multiplicity $\geq 2$ ). In case $n=2$, Ilmanen ([Ilm95]) showed that any self-shrinker corresponding to a tangent flow at the first singular time of a mean curvature flow starting at an embedded, compact surface in $\mathbb{R}^{3}$ has smoothly embedded support. For $n \geq 3$, can a self-shrinker arising as a tangent flow at the first singular time of a mean curvature flow starting at a compact, embedded $n$-dimensional hypersurface develop classical singularities?

(6) Must every branch point of a stationary codimension 1 integral varifold be the limit point of a sequence of classical singularities?

Part 2. An outline of the proof of Theorem 3.3 (Regularity AND COMPACTNESS FOR STABLE CODIMENSION 1 VARIFOLDS WITH NO CLASSICAL SINGULARITIES)

In Sections 6-8 below we give a description of the main ideas involved in the proof of the main regularity and compactness theorem for stable codimension 1 varifolds, i.e. Theorem 3.3. Both the regularity and compactness 
conclusions of this theorem rely on the same set of estimates. Since these estimates are local in nature, to explain the key ideas behind them it suffices to consider the case where the ambient manifold is an open ball of the Euclidean space $\mathbb{R}^{n+1}$, say $B_{2}^{n+1}(0)$; generalizing the results to the case of arbitrary Riemannian ambient manifolds is just a matter of making appropriate technical modifications (to handle the lower order error terms) to the estimates needed in the Euclidean setting. (See [[Wic14a], Section 18] for the full details of how this is done.)

It will be convenient to use the following notation throughout the discussion:

Let $\mathcal{S}$ be the class of stationary, stable varifolds $V \in \operatorname{IV}_{n}\left(B_{2}^{n+1}(0)\right)$ with no classical singularities and with $0 \in \operatorname{spt}\|V\|$.

Let $\mathcal{M}$ be the collection of smoothly embedded hypersurfaces $M$ of $B_{2}^{n+1}(0)$ with (i) locally finite mass, i.e. $\mathcal{H}^{n}(M \cap K)<\infty$ for each compact subset $K$ of $B_{2}^{n+1}(0)$; (ii) no removable singularities, i.e. the property that if $Y \in \bar{M} \cap B_{2}^{n+1}(0)$ and $\bar{M}$ is smoothly embedded in a neighborhood of $Y$, then $Y \in M$; (iii) $0 \in \bar{M}$; (iv) $\operatorname{sing} M=\emptyset$ if $n \leq 6$, $\operatorname{sing} M$ discrete if $n=7$ and $\operatorname{dim}_{\mathcal{H}} \operatorname{sing} M \leq n-7$ if $n \geq 8$, where sing $M=(\bar{M} \backslash M) \cap B_{2}^{n+1}(0)$, and (v) the multiplicity 1 varifold $|M|$ associated with $M$ stationary and stable in $B_{2}^{n+1}(0)$.

It follows directly from the definitions that $\{|M|: M \in \mathcal{M}\} \subset \mathcal{S}$. Furthermore, if $M \in \mathcal{M}$, an easy cut-off function argument shows that each connected component of $M(=\operatorname{reg}|M|)$ is stationary in $B_{2}^{n+1}(0)$. In view of this fact and the Constancy Theorem [[Sim83a], Theorem 41.1], we see that our Theorem 3.3 says that the two classes $\mathcal{S}$ and $\mathcal{M}$ are in fact the same modulo multiplicity, i.e. that

$$
V \in \mathcal{S} \Longleftrightarrow V \in \mathrm{IV}_{n}\left(B_{2}^{n+1}(0)\right) \text { and } \operatorname{reg} V \in \mathcal{M} .
$$

\section{Basic strategy and main steps}

6.1. The Sheeting Theorem and the Minimum Distance Theorem. The following small-excess regularity result for $V \in \mathcal{S}$ (Theorem 6.1) and the disjointness property of the varifold closure of $\mathcal{S}$ from certain cones (Theorem 6.3) are at the heart of the proof of Theorem 3.3:

Theorem 6.1 (Sheeting Theorem; [[Wic14a], Theorem 3.3']). Let $q$ be a positive integer. There exists $\epsilon=\epsilon(n, q) \in(0,1)$ such that if $V \in \mathcal{S}$, $\left(\omega_{n} 2^{n}\right)^{-1}\|V\|\left(B_{2}^{n+1}(0)\right)<q+1 / 2, q-1 / 2 \leq \omega_{n}^{-1}\|V\|\left(\mathbb{R} \times B_{1}^{n}(0)\right)<q+1 / 2$ and

$$
\begin{gathered}
\int_{\mathbb{R} \times B_{1}^{n}(0)}\left|x^{1}\right|^{2} d\|V\|(X)<\epsilon \text { then } \\
V L\left(\mathbb{R} \times B_{1 / 2}^{n}(0)\right)=\sum_{j=1}^{q}\left|\operatorname{graph} u_{j}\right|
\end{gathered}
$$


where $u_{j} \in C^{1, \alpha}\left(B_{1 / 2}^{n}(0) ; \mathbb{R}\right)$ with

$$
\left\|u_{j}\right\|_{C^{1, \alpha}\left(B_{1 / 2}^{n}(0)\right)} \leq C\left(\int_{B_{1}^{n}(0) \times \mathbb{R}}\left|x^{n+1}\right|^{2} d\|V\|(X)\right)^{1 / 2}
$$

for some fixed constants $\alpha=\alpha(n, q) \in(0,1), C=C(n, q) \in(0, \infty)$ and each $j=1,2, \ldots, q$;

$$
u_{1} \leq u_{2} \leq \ldots \leq u_{q}
$$

and $u_{j}$ solving the minimal surface equation weakly on $B_{1 / 2}$ for each $j=$ $1,2, \ldots, q$.

Remark: This theorem is sharp with respect to all of its hypotheses corresponding to the requirement that $V \in \mathcal{S}$. To see this, note that the theorem is false in higher codimension even for area minimizers in view of examples such as $V_{t}=\left\{(z, w) \in \mathbb{C} \times \mathbb{C}: z^{2}=t w^{3}+t w\right\} \cap B_{1}(0)$ which corresponds to an embedded surface in $\mathbb{R}^{4}$ lying close to the plane $\{z=0\} \cap B_{1}(0)$ for small real $t$, and as a complex curve corresponds to a locally area minimizing surface. Rescalings of a standard Catenoid in $\mathbb{R}^{3}$ shows that the theorem is false in the absence of the stability hypothesis. Pairs of transversely intersecting planes close to a plane shows that the theorem is false without the no-classical-singularities hypothesis.

In the special case when $V=|M|$ for some $M \in \mathcal{M}$, Theorem 6.1 was established by Schoen and Simon in [SS81], and this special case plays a crucial role in the proof of Theorem 6.1. The Schoen-Simon version of Theorem 6.1 in fact requires only that $\operatorname{sing} V$ be of locally finite $(n-2)$ dimensional Hausdorff measure, but for our purposes it suffices to know that it holds for $V=|M|$ with $M \in \mathcal{M}$, i.e. the validity of the following:

Theorem 6.2 (Schoen-Simon; Special case of [[SS81], Theorem 2]). Let $q$ be a positive integer. There exists $\epsilon=\epsilon(n, q) \in(0,1)$ such that if $V=|M|$ for some $M \in \mathcal{M}, q-1 / 2 \leq \omega_{n}^{-1}\|V\|\left(\mathbb{R} \times B_{1}^{n}(0)\right)<q+1 / 2$,

$$
\int_{\mathbb{R} \times B_{1}^{n}(0)}\left|x^{n+1}\right|^{2} d\|V\|(X)<\epsilon
$$

then the conclusions of Theorem 6.1 hold. Equivalently, if in Theorem 6.1 we assume in place of the no-classical-singularities hypothesis on $V$ that $\operatorname{sing} V=\emptyset$ in case $n \leq 6, \operatorname{sing} V$ is discrete in case $n=6$ or $\operatorname{dim}_{\mathcal{H}}(\operatorname{sing} V) \leq$ $n-7$ in case $n \leq n-7$, and keep all other assumptions, then the conclusion of Theorem 6.1 holds.

Remarks: (1) It takes a little thought to see the equivalence of the two assertions in Theorem 6.2. The key point is the general fact that if $V \in \operatorname{IV}_{n}\left(B_{2}^{n+1}(0)\right)$ is stationary and $\mathcal{H}^{n-1}(\operatorname{sing} V)=0$, then the multiplicity 1 varifold $|M|$ associated with any connected component $M$ of $\operatorname{reg} V$ is stationary in $B_{2}^{n+1}(0)$. This claim can easily be checked with the help of a straightforward cut-off function argument based on: (i) the volume growth 
bounds implied by the monotonicity formula (1.4) and (ii) the fact that the mean-curvature vanishes on $\operatorname{reg} V$.

(2) The proof of Theorem 6.1 (to be sketched below) does not amount to a direct reduction to Theorem 6.2. Instead, Theorem 6.2 is used, several times, in the linear analysis needed for the proof of Theorem 6.1, as well as in verifying, in an induction argument, the validity of Theorem 6.1 in the absence of points of $V$ with density $\geq q$. In particular, a principal way in which the stability hypothesis enters the proof of Theorem 6.1 is through the use of Theorem 6.2 during the course of its proof.

(3) Theorem 6.2 is an a priori estimate for varifolds $V \in \mathcal{S}$ satisfying the expected regularity. The fact that this a priori estimate, among other things, can be used to establishing the sharp regularity result for varifolds in $\mathcal{S}$ can be thought of as a geometric version of the principle, widely known in various PDE settings, that a priori estimates imply regularity.

For $V \in \mathcal{S}$, Theorem 6.1 implies that a point $Y \in \operatorname{spt}\|V\| \cap B_{2}^{n+1}(0)$ where one tangent cone is supported on an $n$-dimensional plane is a regular point of $V$; it then follows from the Generalized Stratification Theorem (Theorem 2.1) that $\operatorname{dim}_{\mathcal{H}}(\operatorname{sing} V) \leq n-1$. To get closer towards the goal of further reducing the dimension of $\operatorname{sing} V$, one has to rule out next the possibility that a tangent cone at a singularity of $V$ is supported on three or more half-hyperplanes meeting along an $(n-1)$-dimensional subspace. This is achieved with the help of the following theorem:

Theorem 6.3 (Minimum Distance Theorem; [[Wic14a], Theorem 3.4]). Let $\mathbf{C} \in \operatorname{IV}_{n}\left(\mathbb{R}^{n+1}\right)$ be a stationary cone in $\mathbb{R}^{n+1}$ such that spt $\|\mathbf{C}\|$ consists of three or more $n$-dimensional half-hyperplanes meeting along a common $(n-1)$-dimensional subspace. There exists $\epsilon=\epsilon(\mathbf{C}) \in(0,1)$ such that if $V \in \mathcal{S}$ and $\left(\omega_{n} 2^{n}\right)^{-1}\|V\|\left(B_{2}^{n+1}(0)\right)<\Theta(\|\mathbf{C}\|, 0)+1 / 2$ then

$$
\left.\operatorname{dist}_{\mathcal{H}} \text { (spt }\|V\| \cap B_{1}^{n+1}(0) \text {, spt }\|\mathbf{C}\| \cap B_{1}^{n+1}(0)\right)>\epsilon .
$$

Remark: Just like Theorem 6.1, this theorem is sharp with respect to all of the hypotheses corresponding to the requirement $V \in \mathcal{S}$. In particular it is false in higher codimension since for instance we can perturb a pair of two dimensional planes in $\mathbb{R}^{4}$ intersecting along a line into a union of two disjoint planes. It is also false without the stability hypothesis as seen by appropriate rescalings of a Scherk's second surface in $\mathbb{R}^{3}$.

6.2. Proof of Theorem 3.3 using the Sheeting Theorem and the Minimum Distance Theorem. Let $V$ be an $n$-varifold on $B_{2}^{n+1}(0)$ arising as the varifold limit of a sequence of varifolds in $\mathcal{S}$. By the Allard-Almgren compactness theorem (Theorem 1.1), it follows that $V \in \operatorname{IV}_{n}\left(B_{2}^{n+1}(0)\right)$ and that $V$ is stationary in $B_{2}^{n+1}(0)$. By the Sheeting Theorem (Theorem 6.1), it follows that $V$ is stable. And by the Minimum Distance Theorem (Theorem 6.3), it follows that $V$ has no classical singularities. Thus, once Theorems 6.1 and 6.3 are in place, the compactness conclusion of Theorem 3.3 follows. It is then straightforward to obtain the regularity conclusions of 
Theorem 3.3 with the help of a standard tangent cone analysis based on Simons' Theorem (Theorem 3.1) and the Almgren-Federer Generalized Stratification, Theorem 2.1.

Thus the Sheeting Theorem and the Minimum Distance Theorem form the back bone of Theorem 3.3.

6.3. Proof strategy for the Sheeting Theorem and the Minimum Distance Theorem: Induction. Before delving into any of the details, let us first motivate the approach taken in [Wic14a] to proving the Sheeting Theorem and the Minimum Distance Theorem. To do so, consider the case that $V=|M|$ for some $M \in \mathcal{M}$, or more generally, the case that $V \in \mathcal{S}$ with $\operatorname{sing} V$ having locally finite $(n-2)$-dimensional Hausdorff measure. In this case, Theorem 6.3 can be deduced from Theorem 6.1 in a straightforward manner as follows:

First note that in this case, a standard argument shows that $\operatorname{sing} V$ is "removable for the stability inequality (3.2)" in the sense that the inequality $\int_{\text {reg } V}|A|^{2} \zeta^{2} \leq \int_{\text {reg } V}|\nabla \zeta|^{2}$ holds for every $\zeta \in C_{c}^{1}(U)$ (and not just for $\zeta \in C_{c}^{1}(\operatorname{reg} V)$ as (3.2) initially requires). In particular, we may take a standard cut-off function $\zeta$ in this improved stability inequality to deduce that

$$
\int_{M \cap B_{3 / 4}^{n+1}(0)}|A|^{2} \leq C
$$

for a fixed constant $C=C(n, \Lambda)$ independent of $M$, where $\Lambda$ is any upper bound for $\mathcal{H}^{n}\left(M \cap B_{1}^{n+1}(0)\right)$. This, the Cauchy-Schwarz inequality, and the volume growth bounds for $M$ (implied by the monotonicity formula (1.4)) imply that if $L$ is an $(n-1)$-dimensional subspace of $\mathbb{R}^{n+1}$, then for each fixed $\Lambda>0$,

$$
\sup _{M \in \mathcal{M}, \mathcal{H}^{n}\left(M \cap B_{1}^{n+1}(0)\right) \leq \Lambda} \int_{M \cap B_{3 / 4}^{n+1}(0) \cap(L)_{\tau}}|A| \rightarrow 0
$$

as $\tau \rightarrow 0$.

On the other hand, if $\mathbf{C}$ is as in Theorem $6.3, L$ is the singular axis of $\mathbf{C}$, $p: \mathbb{R}^{n+1} \rightarrow L$ is the orthogonal projection, and if, contrary to Theorem 6.3 , there is a sequence $M_{j} \in \mathcal{M}$ with $\omega_{n}^{-1} \mathcal{H}^{n}\left(M_{j} \cap B_{1}^{n+1}(0)\right) \leq \Theta(\|\mathbf{C}\|, 0)+1 / 2$ and $\operatorname{dist}_{\mathcal{H}}\left(M_{j} \cap B_{1 / 2}^{n+1}(0)\right.$, spt $\left.\|\mathbf{C}\| \cap B_{1 / 2}^{n+1}(0)\right) \rightarrow 0$, then for sufficiently large $j, p^{-1}(y) \cap M_{j} \cap(L)_{\tau}$ is a non-empty finite collection of disjoint, smooth embedded curves $\gamma_{j}^{k}, 1 \leq k \leq \ell_{j}$, for $\mathcal{H}^{n-1}$-a.e. $y \in L \cap B_{1 / 4}^{n+1}(0)$, and by Theorem 6.1, if $\nu_{j}$ denotes a choice of unit normal to $M_{j}$, then

$$
\theta \leq \inf _{1 \leq k \leq \ell_{j}}\left|\nu_{j}\left(P_{j}^{k, 1}\right)-\nu_{j}\left(P_{j}^{k, 2}\right)\right| \leq \int_{p^{-1}(y) \cap M_{j} \cap(L)_{\tau}}|A| d \mathcal{H}^{1}
$$

where $P_{j}^{k, 1}, P_{j}^{k, 2}$ are the end points of $\gamma_{j}^{k}$ and $\theta$ is a fixed positive constant determined by spt $\|\mathbf{C}\|$. Integrating over $y \in L \cap B_{1 / 4}^{n+1}(0)$ and using the 
co-area formula, we see that this leads to a contradiction with (6.1) for sufficiently large $j$.

Notice that this simple argument to deduce Theorem 6.3 from Theorem 6.1 clearly breaks down if we only have the no-classical-singularities hypothesis (as in Theorem 3.3) instead of the hypothesis that $\operatorname{sing} V$ is sufficiently low dimensional; indeed, even under the hypothesis $\mathcal{H}^{n-1}(\operatorname{sing} V)=$ 0 which is stronger than the no-classical-singularities hypothesis and which together with Theorem 6.1 makes it possible to justify (6.2) for $\mathcal{H}^{n-1}$-a.e. $y \in L \cap B_{1 / 4}^{n+1}(0)$, the above argument breaks down because in this case, a priori we do not know even whether $\int_{\operatorname{reg} V \cap B_{3 / 4}^{n+1}(0) \cap(L)_{\tau}}|A|<\infty$, let alone the statement (6.1). Thus, one is lead to look for an argument to prove Theorem 6.3 that does not a priori require unconditional integral control of the second fundamental form of reg $V$ in a whole neighborhood of the singular axis of $\mathbf{C}$.

Quite apart from this issue, what is also clear is that a result such as Theorem 6.1 is likely to be necessary (or at least extremely useful) for the proof of Theorem 6.3 since, in order to derive a contradiction from the assumption that there exist $V \in \mathcal{S}$ arbitrarily close to $\mathbf{C}$, we need a way to control the singular set of $V$ in the region outside a small tubular neighbourhood of the singular axis of $\mathbf{C}$. Since the existence of a large set of branch points in varifolds in $\mathcal{S}$ cannot a priori be ruled out, we cannot argue that $\operatorname{sing} V$ must concentrate near $\operatorname{sing} \mathbf{C}$ without the help of a result such as Theorem 6.1.

However, any attempt to prove Theorem 6.1 completely independently of a result in the spirit of Theorem 6.3 does not have much chance of success for the following reason: First, note that obvious examples such as appropriate rescalings of the standard Catenoid show that the stability hypothesis is necessary for Theorem 6.1. Due to the possible presence of a large singular set, there is however no way to make direct use of the stability inequality (unlike in the Schoen-Simon version, i.e. Theorem 6.2). Indeed, without any information on the set of singularities where there is a tangent cone supported on three or more half-spaces meeting along a common axis (which singularities are completely ruled out by Theorem 6.3), under the hypotheses of Theorem 6.1 one has at best regularity of $V$ away from the union of a set $S$ of Hausdorff dimension $\leq n-1$ and the set $S_{q}$ of points with density $\geq q$, where $q$ is as in Theorem 6.1 (this follows by assuming by induction the validity of Theorem 6.1 with any $q^{\prime} \in\{1,2, \ldots, q-1\}$ in place of $q$ ); this, as pointed out before, is nowhere near the amount of control on $\operatorname{sing} V$ necessary to make good use of the stability hypothesis. If however we had Theorem 6.3 at our disposal, then by the Generalised Stratification Theorem (Theorem 2.1) together with Simons' theorem (Theorem 3.1), we may assume regularity of $V$ away from $S_{q}$ and away from a closed set of Hausdorff dimension $\leq n-7$. In particular, by Theorem 6.2 , this would yield Theorem 6.1 at least in case $S_{q} \cap\left(\mathbb{R} \times B_{3 / 4}^{n}\right)=\emptyset$. 
The two theorems are thus closely connected to each other, a fact that appears to create an impasse. This naturally demands a strategy that requires "elevating Theorem 6.3 to equal standing with Theorem 6.1" and aims to prove both theorems simultaneously; this indeed is the approach taken in $[\mathbf{W i c 1 4 a}$ ] where the two theorems are proved simultaneously by an inductive argument, where induction for Theorem 6.1 is on $q$ and for Theorem 6.3 is on the density $\Theta(\|\mathbf{C}\|, 0)$ of the cone $\mathbf{C}$ whose value $\in\{q-1 / 2, q\}$ for some integer $q \geq 2$.

Thus, let $q \geq 2$ be an integer, and assume the following: INDUCTION HYPOTHESES:

(H1) Theorem 6.1 holds with any $q^{\prime} \in\{1, \ldots, q-1\}$ in place of $q$. (H2) Theorem 6.3 holds whenever $\Theta(\|\mathbf{C}\|, 0) \in\{3 / 2, \ldots, q-1 / 2, q\}$.

Completion of induction is achieved by carrying out, assuming (H1), (H2), the following three steps in the order they are listed:

(i) Prove Theorem 6.1;

(ii) prove Theorem 6.3 in case $\Theta(\|\mathbf{C}\|, 0)=q+1 / 2$;

(iii) prove Theorem 6.3 in case $\Theta(\|\mathbf{C}\|, 0)=q+1$.

Note that the base case $q=1$ of Theorem 6.1 is a direct consequence of Allard's regularity theorem (Theorem 1.2), and the case $\Theta(\|\mathbf{C}\|, 0)=3 / 2$ of Theorem 6.3 follows from a theorem of Simon ([Sim93], Theorem 4); incidentally, neither of these requires the stability hypothesis, and they both hold for stationary integral varifolds of arbitrary co-dimension. The case $\Theta(\|\mathbf{C}\|, 0)=2$ of Theorem 6.3 follows by taking $q=1$ in the argument for step (iii) above, and using the case $q=1$ of Theorem 6.1 and the case $\Theta(\|\mathbf{C}\|, 0)=3 / 2$ of Theorem 6.3 in place of the induction hypotheses (H1), $(\mathrm{H} 2)$ respectively.

\subsection{An elementary consequence of the induction hypotheses.}

For $V \in \mathcal{S}$ and $q$ an integer $\geq 2$, let $S_{q}=\{Z: \Theta(\|V\|, Z) \geq q\}$. We shall make use of the following direct consequence of the induction hypotheses (H1), (H2) and Theorem 2.1 a number of times during the course of the completion of induction for the Sheeting and Minimum Distance Theorems:

( $\star$ If the induction hypotheses (H1), (H2) hold, $\Omega$ is an open subset of $B_{2}^{n+1}(0), V \in \mathcal{S}$ and $S_{q} \cap \Omega=\emptyset$, then $\operatorname{sing} V \cap \Omega=\emptyset$ if $n \leq 6$, $\operatorname{sing} V \cap \Omega$ is discrete if $n=7$ and $\operatorname{dim}_{\mathcal{H}}(\operatorname{sing} V \cap \Omega) \leq n-7$ of $n \geq 8$.

In fact we can say more generally the following: For small $\delta \in(0,1)$, if we let

$$
\mathcal{S}_{q, \delta}=\left\{V \in \mathcal{S}:\left(\omega_{n} 2^{n}\right)^{-1}\|V\|\left(B_{2}^{n+1}(0)\right)<q-\delta\right\},
$$

then as a consequence of the monotonicity formula (1.4), the induction hypotheses (H1), (H2) and Theorems 2.1 and 3.1, the following compactness property of $\mathcal{S}_{q, \delta}$ holds: There exists $\sigma=\sigma(n, q, \delta) \in(0,1)$ such that if $V_{1}, V_{2}, V_{3}, \ldots$ belong to $\mathcal{S}_{q, \delta}$, then there exists $V \in \operatorname{IV}_{n}\left(B_{2}^{n+1}(0)\right)$ with 
reg $\eta_{0,2 \sigma^{-1} \#} V \in \mathcal{M}$ such that after passing to a subsequence, $V_{j} \rightarrow V$ as varifolds on $B_{2}^{n+1}(0)$. In particular, reg $\eta_{0,2 \sigma^{-1}} \# \in \mathcal{M}$ for each $V \in \mathcal{S}_{q, \delta}$. Consequently, if $V \in \mathcal{S}$, then $\operatorname{sing} V=S_{q} \cup S$ where $\operatorname{dim}_{\mathcal{H}}(S) \leq n-7(S$ empty if $n \leq 6$ and discrete if $n=6$ ), so that in particular we have $(\star)$ above.

Let us now describe, by elaborating on each of the steps (i), (ii) and (iii) listed above, how the proof strategy described in Section 6.3 above is implemented. Most of the work required, by far, goes into competing step (i).

\section{Step (i): Induction for the Sheeting Theorem}

7.1. Linear theory: proper blow-up classes. Fix an integer $q \geq$ 1. Following [[Wic14a], Section 4], a collection $\mathcal{B}$ of functions $v=$ $\left(v^{1}, v^{2}, \ldots, v^{q}\right): B_{1} \rightarrow \mathbb{R}^{q}$ is said to be a proper blow-up class if it satisfies the following properties for some fixed constant $C \in(0, \infty)$ :

$(\mathcal{B} 1) \mathcal{B} \subset W_{\text {loc }}^{1,2}\left(B_{1} ; \mathbb{R}^{q}\right) \cap L^{2}\left(B_{1} ; \mathbb{R}^{q}\right)$.

$(\mathcal{B} 2)$ If $v \in \mathcal{B}$, then $v^{1} \leq v^{2} \leq \ldots \leq v^{q}$.

$(\mathcal{B} 3)$ If $v \in \mathcal{B}$, then $\Delta v_{a}=0$ in $B_{1}$ where $v_{a}=q^{-1} \sum_{j=1}^{q} v^{j}$.

$(\mathcal{B} 4)$ For each $v \in \mathcal{B}$ and each $z \in B_{1}$, either $(\mathcal{B} 4 I)$ or $(\mathcal{B} 4 I I)$ below is true:

(B 4I) The Hardt-Simon inequality

$$
\sum_{j=1}^{q} \int_{B_{\rho / 2}(z)} R_{z}^{2-n}\left(\frac{\partial\left(\left(v^{j}-v_{a}(z)\right) / R_{z}\right)}{\partial R_{z}}\right)^{2} \leq C \rho^{-n-2} \int_{B_{\rho}(z)}\left|v-\ell_{v, z}\right|^{2}
$$

holds for each $\rho \in\left(0, \frac{3}{8}(1-|z|)\right]$, where $R_{z}(x)=|x-z|$, $\ell_{v, z}(x)=v_{a}(z)+D v_{a}(z) \cdot(x-z)$ and $\left(v-\ell_{v, z}\right)=\left(v^{1}-\right.$ $\left.\ell_{v, z}, v^{2}-\ell_{v, z}, \ldots, v^{q}-\ell_{v, z}\right)$.

$\left(\mathcal{B}_{4} I I\right)$ There exists $\sigma=\sigma(z) \in(0,1-|z|]$ such that $\Delta v=0$ in $B_{\sigma}(z)$. $(\mathcal{B} 5)$ (Invariances) If $v \in \mathcal{B}$, then

$(\mathcal{B} 5 I) \widetilde{v}_{z, \sigma}(\cdot) \equiv\|v(z+\sigma(\cdot))\|_{L^{2}\left(B_{1}(0)\right)}^{-1} v(z+\sigma(\cdot)) \in \mathcal{B}$ for each $z \in B_{1}$ and $\sigma \in\left(0, \frac{3}{8}(1-|z|)\right]$ whenever $v \not \equiv 0$ in $B_{\sigma}(z)$;

$(\mathcal{B} 5 I I) v \circ \gamma \in \mathcal{B}$ for each orthogonal rotation $\gamma$ of $\mathbb{R}^{n}$ and

$(\mathcal{B} 5 I I I)\left\|v-\ell_{v}\right\|_{L^{2}\left(B_{1}(0)\right)}^{-1}\left(v-\ell_{v}\right) \in \mathcal{B}$ whenever $v-\ell_{v} \not \equiv 0$ in $B_{1}$, where $\ell_{v}(x)=v_{a}(0)+D v_{a}(0) \cdot x$ for $x \in \mathbb{R}^{n}$ and $v-\ell_{v}$ is abbreviation for $\left(v^{1}-\ell_{v}, v^{2}-\ell_{v}, \ldots, v^{q}-\ell_{v}\right)$.

$(\mathcal{B} 6)$ (Compactness) If $\left\{v_{k}\right\}_{k=1}^{\infty} \subset \mathcal{B}$ then there exists a subsequence $\left\{k^{\prime}\right\}$ of $\{k\}$ and a function $v \in \mathcal{B}$ such that $v_{k^{\prime}} \rightarrow v$ locally in $L^{2}\left(B_{1}\right)$ and locally weakly in $W^{1,2}\left(B_{1}\right)$.

$\left(\mathcal{B}^{7)}\right.$ (Minimum Distance property) If $v \in \mathcal{B}$ is such that for each $j=1,2, \ldots, q$, there exist linear functions $L_{1}^{j}, L_{2}^{j}: \mathbb{R}^{n} \rightarrow \mathbb{R}$ with $L_{1}^{j}(0, y)=L_{2}^{j}(0, y)$ for $y \in \mathbb{R}^{n-1}, v^{j}\left(x^{2}, y\right)=L_{1}^{j}\left(x^{2}, y\right)$ if $x^{2}<0$ 
and $v^{j}\left(x^{2}, y\right)=L_{2}^{j}\left(x^{2}, y\right)$ if $x^{2} \geq 0$, then $v^{1}=v^{2}=\ldots=v^{q}=L$ for some linear function $L: \mathbb{R}^{n} \rightarrow \mathbb{R}$.

The role that proper blow-up classes play in completing step (i) will become clear in the next section (see Theorem 7.2).

Note that in view of the compactness property $(\mathcal{B} 6)$, the property $\left(\mathcal{B}^{n}\right)$ implies the following: Let $H=\left(H^{1}, H^{2}, \ldots, H^{q}\right): \mathbb{R}^{n} \rightarrow \mathbb{R}^{q}$ be such that $H^{1} \leq H^{2} \leq \ldots \leq H^{q}$, at least two of $H^{1}, H^{2}, \ldots, H^{q}$ are distinct, and for each $j=1,2, \ldots, q$, there exist linear functions $L_{1}^{j}, L_{2}^{j}: \mathbb{R}^{n} \rightarrow \mathbb{R}$ with $L_{1}^{j}(0, y)=L_{2}^{j}(0, y)$ for $y \in \mathbb{R}^{n-1}$ such that $H^{j}\left(x^{2}, y\right)=L_{1}^{j}\left(x^{2}, y\right)$ if $x^{2}>0$ and $H^{j}\left(x^{2}, y\right)=L_{2}^{j}\left(x^{2}, y\right)$ if $x^{2} \leq 0$. Then there exists $\epsilon=\epsilon(H, \mathcal{B}) \in(0,1)$ such that $\int_{B_{1 / 2}}|v-H|^{2} \geq \epsilon$ for each $v \in \mathcal{B}$. This explains why property $\left(\mathcal{B}^{\gamma}\right)$ is called the Minimum Distance property.

The following regularity theorem says that functions in a proper blow-up class are nothing but harmonic functions.

TheOREm 7.1 (Sheeting theorem for proper blow-up classes; [[Wic14a], Theorem 4.1]). If $\mathcal{B}$ is a proper blow-up class for some $C \in(0, \infty)$, then each $v \in \mathcal{B}$ is harmonic in $B_{1}$. Furthermore, if $v \in \mathcal{B}$ and there is a point $z \in B_{1}$ such that (B $4 I)$ is satisfied, then $v^{1}=v^{2}=\ldots=v^{q}$.

Note the significance of property $\left(\mathcal{B}^{7}\right)$ for Theorem 7.1 ; an obvious counterexample to the theorem in the absence of this property is the set of all functions $v=\left(v^{1}, v^{2}\right): B_{1} \rightarrow \mathbb{R}^{2}$ such that $v^{1}=\min \left\{\ell, \ell^{\prime}\right\}$ and $v^{2}=\max \left\{\ell, \ell^{\prime}\right\}$ for some affine functions $\ell, \ell^{\prime}: \mathbb{R}^{n} \rightarrow \mathbb{R}$ with $\|\ell\|_{L^{2}\left(B_{1}\right)},\left\|\ell^{\prime}\right\|_{L^{2}\left(B_{1}\right)} \leq 1$.

Brief Sketch of proof of Theorem 7.1. For $v \in \mathcal{B}$, let $\Gamma_{v}$ denote the set of points $z \in B_{1}$ such that property $(\mathcal{B} 4 I)$ holds and yet there is no neighborhood of $z$ in which values of $v^{1}, v^{2}, \ldots, v^{q}$ all agree almost everywhere (and coincide with the harmonic function $v_{a}$ ). Then $\Gamma_{v}$ is a closed subset of $B_{1}$ and by property $(\mathcal{B} 4 I I), \Delta v^{j}=0$ in $B_{1} \backslash \Gamma_{v}$ for each $j=1,2, \ldots, q$. Theorem 7.1 is then equivalent to the statement that $\Gamma_{v}=\emptyset$.

If $\mathcal{B} \subset C^{1}\left(B_{1}\right)$, then Theorem 7.1 follows immediately; indeed in this case, it is easy to see that at every point $z \in B_{1}$ where $(\mathcal{B} 4 I)$ holds, we have that $v^{j}(z)=v_{a}(z)$ for each $j=1,2, \ldots, q$ so in particular, $\Gamma_{v} \neq B_{1}$. If now $\Gamma_{v} \neq \emptyset$, then we may choose a point $y \in B_{1} \backslash \Gamma_{v}$ such that $\operatorname{dist}\left(y, \Gamma_{v}\right)<\operatorname{dist}\left(y, \partial B_{1}\right)$ and apply the Hopf boundary point lemma in the ball $B_{\operatorname{dist}\left(y, \Gamma_{v}\right)}(y)$ to the function $v^{j}-v^{j-1}$ for each $j=1,2, \ldots, q$ to get a contradiction.

To prove that $\mathcal{B} \subset C^{1}\left(B_{1}\right)$, in view of a general Campanato type lemma ([[Wic14a], Lemma 4.3]), property $(\mathcal{B} 4)$ and property $(\mathcal{B} 5 I)$, it suffices to show that there are constants $\beta=\beta(\mathcal{B}) \in(0, \infty)$ and $\mu=\mu(\mathcal{B}) \in(0,1)$ such that for each $z \in \Gamma_{v} \cap B_{3 / 4}$ and $0<\sigma<\rho / 2 \leq 1 / 8$, 


$$
\sigma^{-n-2} \sum_{j=1}^{q} \int_{B_{\sigma}(z)}\left|v^{j}-\ell_{z}\right|^{2} \leq \beta\left(\frac{\sigma}{\rho}\right)^{\mu} \rho^{-n-2} \sum_{j=1}^{q} \int_{B_{\rho}(z)}\left|v^{j}-\ell_{z}\right|^{2}
$$

where $\ell_{z}$ is the affine function defined by $\ell_{z}(x)=v_{a}(z)+D v_{a}(z) \cdot(x-z)$. This estimate follows from a "hole-filling" argument based on the HardtSimon inequality (see [[Wic14a], Theorem 4.1]) provided we can show that any homogeneous degree 1 element $\varphi=\left(\varphi^{1}, \varphi^{2}, \ldots, \varphi^{q}\right) \in \mathcal{B}$ is given by a single linear function in the sense that $\varphi^{1}=\varphi^{2}=\ldots=\varphi^{q}=L$ for some linear function $L$.

To classify homogeneous degree 1 elements in $\mathcal{B}$, note first that the directions of translation invariance of any such element $\varphi \in \mathcal{B}$ form a linear subspace $T(\varphi)$ of $\mathbb{R}^{n}$, and if $\operatorname{dim} T(\varphi) \in\{n, n-1\}$ it follows (from $\left(\mathcal{B}^{7}\right)$ ) that $\varphi$ is given by a linear function. To complete the classification, choose a homogeneous degree 1 element $\varphi \in \mathcal{B}$ with the largest value of $\operatorname{dim} T(\varphi)$ subject to $\operatorname{dim} T(\varphi) \leq n-2$. If $\Gamma_{\varphi} \subset T(\varphi)$, then $\varphi$ is harmonic in $\mathbb{R}^{n} \backslash T(\varphi)$ and since $\varphi \in W_{\text {loc }}^{1,2}\left(\mathbb{R}^{n}\right)$ and translation invariant along $T(\varphi)$, it follows that $\varphi$ is harmonic on $\mathbb{R}^{n}$ and therefore that $\varphi^{1}=\varphi^{2}=\ldots=\varphi^{q}=L$ for some linear function $L$ contrary to the assumption that $\operatorname{dim} T(\varphi) \leq n-2$. If on the other hand $\Gamma_{\varphi} \backslash T(\varphi) \neq \emptyset$, then we can use first the hole-filling argument and the Campanato type lemma referred to above to show that $\varphi$ is $C^{1}$ away from $T(\varphi)$, and consequently the Hopf boundary point lemma as before to obtain a contradiction. See [[Wic14a], Proposition 4.2] for details.

7.2. Coarse blow-ups of stationary varifolds. Let $q$ be a positive integer. Let $\left\{V_{k}\right\}$ be a sequence of stationary integral $n$-varifolds on $B_{2}^{n+1}(0)$ such that

$\left(\omega_{n} 2^{n}\right)^{-1}\left\|V_{k}\right\|\left(B_{2}^{n+1}(0)\right)<q+1 / 2 ; \quad q-1 / 2 \leq \omega_{n}^{-1}\left\|V_{k}\right\|\left(\mathbb{R} \times B_{1}\right)<q+1 / 2$

for each $k=1,2,3, \ldots$, and $\hat{E}_{k} \rightarrow 0$, where $\hat{E}_{k}$ is the height-excess of $V_{k}$ relative the the plane $\left\{x^{1}=0\right\}$, defined by

$$
\hat{E}_{k}^{2} \equiv \hat{E}_{V_{k}}^{2}=\int_{\mathbb{R} \times B_{1}}\left|x^{1}\right|^{2} d\left\|V_{k}\right\|(X) .
$$

Let $\sigma \in(0,1)$. By applying Almgren's approximate graph decomposition theorem ([[Alm83], Corollary 3.11]; see also [[Wic14a], Theorem 5.1]), we obtain for each sufficiently large $k$, Lipschitz functions $u_{k}^{j}: B_{\sigma} \rightarrow \mathbb{R}$, $j=1,2, \ldots, q$, with $u_{k}^{1} \leq u_{k}^{2} \leq \ldots \leq u_{k}^{q}$ and

$$
\operatorname{Lip} u_{k}^{j} \leq 1 / 2 \text { for each } j \in\{1,2, \ldots, q\}
$$

and a measurable subset $\Sigma_{k}$ of $B_{\sigma}$ such that

$$
\operatorname{spt}\left\|V_{k}\right\| \cap\left(\mathbb{R} \times\left(B_{\sigma} \backslash \Sigma_{k}\right)\right)=\cup_{j=1}^{q} \operatorname{graph} u_{k}^{j} \cap\left(\mathbb{R} \times\left(B_{\sigma} \backslash \Sigma_{k}\right)\right)
$$


and

$$
\left\|V_{k}\right\|\left(\mathbb{R} \times \Sigma_{k}\right)+\mathcal{H}^{n}\left(\Sigma_{k}\right) \leq C \hat{E}_{k}^{2}
$$

where $C=C(n, q, \sigma) \in(0, \infty)$. Set

$$
v_{k}^{j}(x)=\hat{E}_{k}^{-1} u_{k}^{j}(x)
$$

for $x \in B_{\sigma}$, and write $v_{k}=\left(v_{k}^{1}, v_{k}^{2}, \ldots, v_{k}^{q}\right)$. Then $v_{k}$ is Lipschitz on $B_{\sigma}$; and by $(7.2)$ and (7.3),

$$
\int_{B_{\sigma}}\left|v_{k}\right|^{2} \leq C, \quad C=C(n, q, \sigma) \in(0, \infty) .
$$

Furthermore, by taking $X=x^{1} \zeta^{1} e^{1}$ for a suitable choice of $\zeta \in C_{c}^{1}\left(\mathbb{R} \times B_{1}\right)$ in the first variation formula (1.2) with $V_{k}$ in place of $V$ and a straightforward computation, we also have

$$
\int_{B_{\sigma}}\left|D v_{k}\right|^{2} \leq C, \quad C=C(n, q, \sigma) \in(0, \infty) .
$$

In view of the arbitrariness of $\sigma \in(0,1)$, by (7.5), (7.6), Rellich's theorem and a diagonal sequence argument, we obtain a function $v \in W_{\text {loc }}^{1,2}\left(B_{1} ; \mathbb{R}^{q}\right) \cap$ $L^{2}\left(B_{1} ; \mathbb{R}^{q}\right)$ and a subsequence $\left\{k_{j}\right\}$ of $\{k\}$ such that $v_{k_{j}} \rightarrow v$ as $j \rightarrow \infty$ in $L^{2}\left(B_{\sigma} ; \mathbb{R}^{q}\right)$ and weakly in $W^{1,2}\left(B_{\sigma} ; \mathbb{R}^{q}\right)$ for every $\sigma \in(0,1)$.

Definitions: (1) Coarse blow-ups: Let $v \in W_{\mathrm{loc}}^{1,2}\left(B_{1} ; \mathbb{R}^{q}\right) \cap L^{2}\left(B_{1} ; \mathbb{R}^{q}\right)$ correspond, in the manner described above, to (a subsequence of) a sequence $\left\{V_{k}\right\}$ of stationary integral $n$-varifolds on $B_{2}^{n+1}(0)$ satisfying (7.1) and with $\hat{E}_{k} \rightarrow 0$, where $\hat{E}_{k}$ is as in (7.2). We shall call $v$ a coarse blow-up of the sequence $\left\{V_{k}\right\}$.

(2) The Class $\mathcal{B}_{q}$ : Denote by $\mathcal{B}_{q}$ the collection of all coarse blow-ups of sequences of varifolds $\left\{V_{k}\right\} \subset \mathcal{S}$ satisfying (7.1) and for which $\hat{E}_{k} \rightarrow 0$, where $\hat{E}_{k}$ is as in $(7.2)$.

A substantial part of $[\mathbf{W i c 1 4 a}]$ is devoted to proving the following:

TheOREM 7.2. If the induction hypotheses (H1), (H2) hold, then $\mathcal{B}_{q}$ is a proper blow-up class.

We shall outline the proof of this claim in sections 7.3-7.6 below.

Note that in case $q=1$ (which corresponds to Allard's regularity theorem, Theorem 1.2, in codimension 1), given (7.2) and (7.3), it is not difficult to see in view of the identity

$$
\int \nabla x^{1} \cdot \nabla \zeta d\left\|V_{k}\right\|=0 \quad \forall \zeta \in C_{c}^{1}\left(\mathbb{R} \times B_{1}\right),
$$

which follows directly from the first variation formula (1.3), that $v=\lim v_{k}$ is harmonic in $B_{1}$, where $v, v_{k}$ are as above (and are real valued). In this case no stability hypothesis on $V_{k}$ nor any information about the singular sets $\operatorname{sing} V_{k}$ is required to draw this conclusion, and the blowing-up process $v_{k} \rightarrow v$ can be thought of as a linearizing procedure of the stationary varifolds at the flat varifold $\left|B_{1}(0)\right|$. Using standard regularity estimates 
for harmonic functions, (7.2) and (7.3), one can directly conclude from this that there exist $\theta=\theta(n) \in(0,1 / 4)$ and $\epsilon=\epsilon(n) \in(0,1)$ such that if $V \in \mathrm{IV}_{n}\left(\mathbb{R} \times B_{1}\right)$ is stationary and the mass bounds (7.1) hold with $V$ in place of $V_{k}$ and $q=1$, and if $\hat{E}_{V}<\epsilon$, then for a suitable choice of an affine $n$-dimensional plane $L$,

$$
\theta^{-n-2} \int_{\mathbb{R} \times B_{\theta}(0)} \operatorname{dist}^{2}(X, L) d\|V\| \leq \frac{1}{2} \hat{E}_{V}^{2}
$$

i.e. that the height-excess of $V$ at scale $\theta$ relative a new reference plane $L$ improves by a factor $1 / 2$ if it is sufficiently small relative to some plane at scale 1. This fact, first established by De Giorgi in the setting of codimension 1 area minimizing boundaries, is at the heart of Allard's regularity theorem, and its iterative application (rotating $V$ slightly at each stage) leads to interior regularity of $V$ when $\hat{E}_{V}$ is sufficiently small.

Thus, having a suitable regularity theory for the linearized problem is crucial to this scheme, and in the present case of varifolds $V \in \mathcal{S}$ satisfying the mass bounds (7.1) for some $q \geq 2$ and having small excess $\hat{E}_{V}$, Theorem 7.1 and Theorem 7.2 provide this theory, subject to the induction hypotheses $(H 1),(H 2)$.

7.3. Elementary properties of coarse blow-ups. The discussion above establishes properties $(\mathcal{B} 1)$ and $(\mathcal{B} 2)$ for $\mathcal{B}=\mathcal{B}_{q}$. It is a straightforward consequence of the first variation formula that property $(\mathcal{B} 3)$ holds for $\mathcal{B}_{q}$. Properties $(\mathcal{B} 5)$ and $(\mathcal{B} 6)$ for $\mathcal{B}_{q}$ are elementary consequences of the estimates (7.5) and (7.6) and the scaling, translation and rotation invariance of stationary varifolds. See [[Wic14a], Section 8] for details. In particular, these properties are very general and do not require the stability hypothesis nor the no-classical-singularities hypothesis on the varifolds giving rise to coarse blow-ups.

To show that property $\left(\mathcal{B}_{4}\right)$ holds for $\mathcal{B}_{q}$ (with a constant $C$ depending only on $n$ and $q$ ), one argues as follows: Fix $v \in \mathcal{B}_{q}$ and $z \in B_{1}$. If

$$
v-\ell_{v, z} \equiv\left(v^{1}-\ell_{v, z}, v^{2}-\ell_{v, z}, \ldots, v^{q}-\ell_{v, z}\right)=0
$$

there is nothing further to prove, so assume $v-\ell_{v, z} \neq 0$. Then by $(\mathcal{B} 5 I I I)$, $\widetilde{v}=\left\|v-\ell_{v, z}\right\|_{L^{2}\left(B_{1}\right)}^{-1}\left(v-\ell_{v, z}\right) \in \mathcal{B}_{q}$. Let $\left\{V_{k}\right\} \subset \mathcal{S}$ be a sequence of varifolds whose coarse blow-up is $\widetilde{v}$. Consider the following two alternatives, one of which must hold:

(1) there exists $\sigma>0$ such that for all sufficiently large $k, Z \in$ $\mathbb{R} \times B_{\sigma}(z) \Longrightarrow \Theta\left(\left\|V_{k}\right\|, Z\right)<q$

(2) there exists a subsequence $\left\{k^{\prime}\right\}$ of $\{k\}$ and points $Z_{k^{\prime}}=\left(z_{k^{\prime}}^{1}, z_{k^{\prime}}^{\prime}\right) \in$ spt $\left\|V_{k^{\prime}}\right\|$ with

$$
\Theta\left(\left\|V_{k^{\prime}}\right\|, Z_{k^{\prime}}\right) \geq q
$$

such that $z_{k^{\prime}}^{\prime} \rightarrow z$.

If (1) holds, then by $(\star)$ we have that for all sufficiently large $k$, $\operatorname{sing} V_{k}\left\llcorner\left(\mathbb{R} \times B_{\sigma}(z)\right)=\emptyset\right.$ if $n \leq 6, \operatorname{sing} V_{k}\left\llcorner\left(\mathbb{R} \times B_{\sigma}(z)\right)\right.$ is discrete if 
$n=6$ and $\operatorname{dim}_{\mathcal{H}}\left(\operatorname{sing} V_{k}\left\llcorner\left(\mathbb{R} \times B_{\sigma}(z)\right)\right) \leq n-7\right.$ if $n \geq 7$. Thus in this case, we may apply Theorem 6.2 to conclude that for all sufficiently large $k$, $V_{k}\left\llcorner\left(\mathbb{R} \times B_{\sigma / 2}(z)\right)\right.$ is given by $q$ (weakly) ordered graphs of smooth functions on $B_{\sigma / 2}(z)$ solving the minimal surface equation. Hence in this case we conclude with the help of standard elliptic estimates that the alternative $(\mathcal{B} 4 I I)$ must hold.

If on the other hand (2) holds, we can use the following argument due to Hardt-Simon ([HS79]): For any fixed $\rho \in\left(0, \frac{3}{8}(1-|z|)\right]$ apply the monotonicity formula (1.4) with $\eta_{Z_{k^{\prime}}, \rho \#} V_{k^{\prime}}$ in place of $V$ and 0 in place of $Y$, and use the condition $\Theta\left(\left\|V_{k^{\prime}}\right\|, Z_{k^{\prime}}\right) \geq q$ to deduce that for all sufficiently large values of $k^{\prime}$,

$$
\begin{aligned}
& \sum_{j=1}^{q} \int_{B_{\rho / 2}\left(z_{k^{\prime}}^{\prime}\right) \backslash \Sigma_{k^{\prime}}}\left(\frac{R_{z_{k^{\prime}}^{\prime}}^{2}}{\left(u_{k^{\prime}}^{j}-z_{k^{\prime}}^{1}\right)^{2}+R_{z_{k^{\prime}}}^{2}}\right)^{\frac{n+2}{2}} \\
& \times R_{z_{k^{\prime}}^{\prime}}^{2-n}\left(\frac{\partial\left(\left(u_{k^{\prime}}^{j}-z_{k^{\prime}}^{1}\right) / R_{z_{k^{\prime}}^{\prime}}\right)}{\partial R_{z_{k^{\prime}}^{\prime}}}\right)^{2} d \mathcal{H}^{n}(x) \\
& \leq C \rho^{-n-2} \int_{\mathbb{R} \times B_{\rho}\left(z_{k^{\prime}}^{\prime}\right)}\left|x^{1}\right|^{2} d\left\|V_{k^{\prime}}\right\|(X),
\end{aligned}
$$

where $C=C(n, q) \in(0, \infty) ; u_{k}, \Sigma_{k}$ are as in (7.2) and (7.2); for any fixed $y \in \mathbb{R}^{n}, R_{y}(x)=|x-y|$ and $\frac{\partial}{\partial R_{y}}$ denotes the radial derivative $\frac{x-y}{R_{y}} \cdot D$. Dividing both sides of the above by $\hat{E}_{k^{\prime}}^{2}$ and passing to the limit yields the Hardt-Simon inequality as in $(\mathcal{B} 4 I)$. See $[[\mathbf{W i c 1 4 a}]$, Section 8] for details.

It takes substantially more effort to establish that $\mathcal{B}_{q}$ satisfies the remaining property $(\mathcal{B} 7)$ (the Minimum Distance Property). We now outline (in Sections 7.4-7.6 below) the key points of the argument needed to achieve this.

\subsection{Minimum Distance Property for coarse blow-ups: Part I.}

7.4.1. Non-concentration of tilt-excess for stationary varifolds. The following lemma establishes a certain a priori estimate for stationary integral $n$-varifolds $V$ on $B_{2}^{n+1}(0)$ with small height-excess relative to a plane $P$. This estimate is inspired by Simon's estimates ([Sim93]; see Lemma 7.8 below) referred to in Section 2.3 above, and says that if the density ratio of $V$ at unit scale is between $q-1 / 2$ and $q+1 / 2$ and if there are points of spt $\|V\|$ with density $\geq q$ "evenly distributed" in a certain precise sense near an $(n-1)$-dimensional subspace $L$, then the tilt-excess of $V$ relative to $P$ in a small neighborhood of $L$ is at most a small fraction of the total tilt-excess of $V$. In [Wic14a], this result is used in the proof that $\mathcal{B}_{q}$ satisfies property $\left(\mathcal{B}^{7}\right)$ and it may also be of independent interest. The precise claim, assuming without loss of generality that $P=\{0\}) \times \mathbb{R}^{n}$, is as follows: 
Lemma 7.3 ([Wic14a], Theorem 7.1). Let $q$ be a positive integer, $\tau \in$ $(0,1 / 16)$ and $\mu \in(0,1)$. There exists a number $\epsilon_{1}=\epsilon_{1}(n, q, \tau, \mu) \in(0,1 / 2)$ such that if $V$ is a stationary integral n-varifold on $B_{2}^{n+1}(0)$ with

$$
\begin{gathered}
\left(\omega_{n} 2^{n}\right)^{-1}\|V\|\left(B_{2}^{n+1}(0)\right)<q+1 / 2, \quad q-1 / 2 \leq \omega_{n}^{-1}\|V\|\left(\mathbb{R} \times B_{1}\right)<q+1 / 2 \text { and } \\
\int_{\mathbb{R} \times B_{1}}\left|x^{1}\right|^{2} d\|V\|(X) \leq \epsilon_{1},
\end{gathered}
$$

and if $L$ is an $(n-1)$-dimensional subspace of $\{0\} \times \mathbb{R}^{n}$ such that

$$
\begin{gathered}
L \cap B_{1 / 2} \subset(\{Z \in \operatorname{spt}\|V\|: \Theta(\|V\|, Z) \geq q\})_{\tau}, \text { then } \\
\int_{(L)_{\tau} \cap\left(\mathbb{R} \times B_{1 / 2}\right)}\left|\nabla^{V} x^{1}\right|^{2} d\|V\|(X) \leq C \tau^{1-\mu} \int_{\mathbb{R} \times B_{3 / 4}}\left|\nabla x^{1}\right|^{2} d\|V\|(X)
\end{gathered}
$$

and consequently,

$$
\int_{(L)_{\tau} \cap\left(\mathbb{R} \times B_{1 / 2}\right)}\left|\nabla^{V} x^{1}\right|^{2} d\|V\|(X) \leq C \tau^{1-\mu} \int_{\mathbb{R} \times B_{1}}\left|x^{1}\right|^{2} d\|V\|(X) .
$$

Here $C=C(n, q, \mu) \in(0, \infty)$, so in particular $C$ is independent of $\tau$, and for a subset $A$ of $\mathbb{R}^{n+1}$, we use the notation $(A)_{\tau}=\left\{X \in \mathbb{R}^{n+1}\right.$ : $\operatorname{dist}(X, A) \leq$ $\tau\}$.

We refer the reader to [[Wic14a], Section 7] for a proof of this lemma. Remark: Note that no stability hypothesis is needed for the above result. Although we have stated the result assuming that the codimension of $V$ is one and the dimension of $L$ is $(n-1)$, it holds, with essentially the same proof (see [[Wic14a], Section 7]), for stationary integral $n$-varifolds of arbitrary codimension and whenever $\operatorname{dim} L=m$ for any $m \in\{0,1,2, \ldots, n-1\}$. In this generality, if the ambient space is the ball $B_{2}^{n+k}(0) \subset \mathbb{R}^{n+k}$, the heightexcess term $\int_{\mathbb{R} \times B_{1}}\left|x^{1}\right|^{2} d\|V\|(X)$ and the tilt-excess terms

$$
\int_{(L)_{\tau} \cap\left(\mathbb{R} \times B_{1 / 2}\right)}\left|\nabla x^{1}\right|^{2} d\|V\|(X), \quad \int_{\mathbb{R} \times B_{3 / 4}}\left|\nabla x^{1}\right|^{2} d\|V\|(X)
$$

take the form

$$
\int_{\mathbb{R}^{k} \times B_{1}} \sum_{j=1}^{k}\left|x^{j}\right|^{2} d\|V\|(X) \quad\left(=\int_{\mathbb{R}^{k} \times B_{1}} \operatorname{dist}^{2}\left(X,\{0\} \times \mathbb{R}^{n}\right) d\|V\|(X)\right)
$$

and

$$
\begin{aligned}
& \int_{(L)_{\tau} \cap\left(\mathbb{R}^{k} \times B_{1 / 2}\right)} \sum_{j=1}^{k}\left|\nabla x^{j}\right|^{2} d\|V\|(X) \\
&\left(=\int_{(L)_{\tau} \cap\left(\mathbb{R}^{k} \times B_{1 / 2}\right)}\left\|p_{T_{X} V}-p_{\{0\} \times \mathbb{R}^{n}}\right\|^{2} d\|V\|(X)\right),
\end{aligned}
$$




$$
\begin{aligned}
\int_{\mathbb{R}^{k} \times B_{3 / 4}} \sum_{j=1}^{k}\left|\nabla x^{j}\right|^{2} d\|V\|(X) & \\
& \left(=\int_{\mathbb{R}^{k} \times B_{3 / 4}}\left\|p_{T_{X} V}-p_{\{0\} \times \mathbb{R}^{n}}\right\|^{2} d\|V\|(X)\right)
\end{aligned}
$$

respectively, where $p_{S}$ denotes the orthogonal projection of $\mathbb{R}^{n+k}$ on to the subspace $S$; moreover, one can take $\tau^{n-m-\mu}$ in place of $\tau^{1-\mu}$ on the right hand side of the inequalities in the conclusion.

7.4.2. To outline next the proof that $\mathcal{B}_{q}$ satisfies property $\left(\mathcal{B}^{\eta}\right)$ (the Minimum Distance property), let $v \in \mathcal{B}_{q}$ and let $L_{1}^{j}, L_{2}^{j}: \mathbb{R}^{n} \rightarrow \mathbb{R}, 1 \leq j \leq q$, be linear functions such that $v^{j}\left(x^{2}, y\right)=L_{1}^{j}\left(x^{2}, y\right) \quad$ if $\quad x^{2}>0 \quad$ and $\quad v^{j}\left(x^{2}, y\right)=L_{2}^{j}\left(x^{2}, y\right) \quad$ if $\quad x^{2} \leq 0$.

Note that then for $1 \leq j \leq q-1, L_{1}^{j} \leq L_{1}^{j+1}$ on $\left\{x^{2} \geq 0\right\}$ and $L_{2}^{j} \leq L_{2}^{j+1}$ on $\left\{x^{2} \leq 0\right\}$. Assume without loss of generality that $L_{1}^{j}(0, y)=L_{2}^{j}(0, y)=0$ for $1 \leq j \leq q$.

We wish to show that $v$ is given by $q$ copies of a single linear function everywhere, i.e. that there is a linear function $L$ such that $v^{j}=L$ for each $j=1,2, \ldots, q$. The proof involves two cases, described separately in the next two sections.

7.5. Minimum Distance Property for coarse blow-ups: Part II. Let $v$ be as in 7.4.2. We first establish the easy case of the Minimum Distance Property for $\mathcal{B}_{q}$, namely, that if all $q$ linear functions defining $v$ on one of the two sides of the hyperplane $\left\{x^{2}=0\right\}$ agree, then $v$ is given by $q$ copies of a single linear function everywhere, i.e. the following:

Lemma 7.4 ([[Wic14a], Lemma 9.1]). Let $v=\left(v^{1}, v^{2}, \ldots, v^{q}\right)$ be as in 7.4.2. If either $L_{1}^{1}=L_{1}^{2}=\ldots=L_{1}^{q}$ or $L_{2}^{1}=L_{2}^{2}=\ldots=L_{2}^{q}$, then there exists a linear function $L$ such that $v^{j}=L$ for each $j=1,2, \ldots, q$.

Proof. Assume without loss of generality that $L_{1}^{1}=L_{1}^{2}=\ldots=L_{1}^{q}=0$. Note that in view of property $\left(\mathcal{B}_{3} 3\right)$, it suffices to show that $L_{2}^{1}=L_{2}^{2}=\ldots=$ $L_{2}^{q}$, so assume, for a contradiction, that

$$
L_{2}^{j} \neq L_{2}^{j+1} \quad \text { for some } \quad j \in\{1,2, \ldots, q-1\} .
$$

Let $\left\{V_{k}\right\} \subset \mathcal{S}$ be a sequence of varifolds whose coarse blow-up is $v$ and let $\tau \in(0,1 / 8)$ be arbitrary. Assumption ( $\dagger$ ) implies, by the argument establishing property $\left(\mathcal{B}_{4}\right)$ for $\mathcal{B}_{q}$, that for all sufficiently large $k, Z \in$ $\left(\mathbb{R} \times B_{1}\right) \cap\left\{x^{2}>\tau\right\} \Longrightarrow \Theta\left(\left\|V_{k}\right\|, Z\right)<q$, so by $(\star)$ and Theorem 6.2, it follows that $V_{k}\left\llcorner\left(\left(\mathbb{R} \times B_{9 / 16}\right) \cap\left\{x^{2}>\tau / 4\right\}\right)\right.$ is given by ordered graphs of smooth functions with small gradients solving the minimal surface equation 
over $\left(\{0\} \times B_{9 / 16}\right) \cap\left\{x^{2}>\tau / 4\right\}$. Thus, if $u_{k}^{j}$ are as in Section 7.2, then (7.8)

$V_{k}\left\llcorner\left(\left(\mathbb{R} \times B_{9 / 16}\right) \cap\left\{x^{2}>\tau / 4\right\}\right)=\sum_{j=1}^{q}\left|\operatorname{graph} u_{k}^{j}\right| L\left(\left(\mathbb{R} \times B_{9 / 16}\right) \cap\left\{x^{2}>\tau / 4\right\}\right)\right.$

where $u_{k}^{j}$ are $C^{\infty}$ on $B_{9 / 16} \cap\left\{x^{2}>\tau / 4\right\}$, solve the minimal surface equation there and satisfy

$$
\sup _{B_{1 / 2} \cap\left\{x^{2}>\tau / 4\right\}}\left|D^{\ell} u_{k}\right|^{2} \leq C_{\tau} \hat{E}_{k}^{2}
$$

for $\ell=0,1,2$, where $C_{\tau}$ is a constant depending only on $n$ and $\tau$, and $D^{\ell}$ denotes the order $\ell$ differentiation.

To derive the necessary contradiction, take now $\psi(X)=\widetilde{\zeta}(X) e^{2}$ in the first variation formula (1.3) to deduce that

$$
\int \nabla^{V_{k}} x^{2} \cdot \nabla^{V_{k}} \widetilde{\zeta}(X) d\left\|V_{k}\right\|(X)=0
$$

for each $k=1,2, \ldots$ and each $\widetilde{\zeta} \in C_{c}^{1}\left(\mathbb{R} \times B_{1}\right)$. Choosing $\widetilde{\zeta}$ to agree with $\zeta^{\prime}\left(x^{1}, x^{\prime}\right)=\zeta\left(x^{\prime}\right)$ in a neighborhood of spt $\left\|V_{k}\right\| \cap\left(\mathbb{R} \times B_{1 / 4}\right)$, where $\zeta \in C_{c}^{1}\left(B_{1 / 4}\right)$ is arbitrary, this leads to the identity

$$
\begin{aligned}
& \sum_{j=1}^{q} \int_{B_{1 / 4}} \sqrt{1+\left|D u_{k}^{j}\right|^{2}}\left(D_{2} \zeta-\frac{D_{2} u_{k}^{j}\left(D \zeta \cdot D u_{k}^{j}\right)}{1+\left|D u_{k}^{j}\right|^{2}}\right)=F_{k}, \quad \text { where } \\
& F_{k}=-\int_{\mathbb{R} \times\left(B_{1 / 4} \cap \Sigma_{k}\right)} \nabla^{V_{k}} x^{2} \cdot \nabla^{V_{k}} \widetilde{\zeta}(X) d\left\|V_{k}\right\|(X) \\
& \quad+\sum_{j=1}^{q} \int_{B_{1 / 4} \cap \Sigma_{k}} \sqrt{1+\left|D u_{k}^{j}\right|^{2}}\left(D_{2} \zeta-\frac{D_{2} u_{k}^{j}\left(D \zeta \cdot D u_{k}^{j}\right)}{1+\left|D u_{k}^{j}\right|^{2}}\right)
\end{aligned}
$$

with $\Sigma_{k}$ as in Section 7.2. Since $\int_{B_{1 / 4}} D_{2} \zeta=0$, it follows from (7.11) that

$$
\sum_{j=1}^{q} \int_{B_{1 / 4}} \frac{\left|D u_{k}^{j}\right|^{2}}{1+\sqrt{1+\left|D u_{k}^{j}\right|^{2}}} D_{2} \zeta-\frac{D_{2} u_{k}^{j}\left(D \zeta \cdot D u_{k}^{j}\right)}{\sqrt{1+\left|D u_{k}^{j}\right|^{2}}}=F_{k}
$$

It is not difficult to check using (7.8), (7.9) and the definition of $\Sigma_{k}$ (see [[Wic14a], Theorem 5.1]) that

$$
B_{1 / 4} \cap \Sigma_{k} \subset B_{1 / 4} \cap\left\{x^{2}<\tau / 2\right\},
$$

and also that for all sufficiently large $k$,

$$
\begin{aligned}
&\left\|V_{k}\right\|\left(\mathbb{R} \times\left(B_{1 / 4}\right.\right.\left.\left.\cap \Sigma_{k}\right)\right)+\mathcal{H}^{n}\left(B_{1 / 4} \cap \Sigma_{k}\right) \\
& \leq C \int_{\left(\mathbb{R} \times B_{1 / 2}\right) \cap\left\{x^{2}<\tau\right\}}\left|\nabla^{V_{k}} x^{1}\right|^{2} d\left\|V_{k}\right\|(X)
\end{aligned}
$$


where $C \in(0, \infty)$ is a fixed constant depending only on $n$ and $q$. Moreover, it follows from the fact that $\hat{E}_{k}^{-1} u_{k} \rightarrow 0$ in $L^{2}$ on $B_{9 / 16} \cap\left\{x^{2} \leq-\tau / 2\right\}$ that

$$
\hat{E}_{k}^{-2} \int_{\left(\mathbb{R} \times B_{9 / 16}\right) \cap\left\{x^{2} \leq-\tau / 2\right\}}\left|x^{1}\right|^{2} d\left\|V_{k}\right\|(X) \rightarrow 0
$$

and consequently, by (1.3), that

$$
\hat{E}_{k}^{-2} \int_{\left(\mathbb{R} \times B_{1 / 2}\right) \cap\left\{x^{2} \leq-\tau\right\}}\left|\nabla^{V_{k}} x^{1}\right|^{2} d\left\|V_{k}\right\|(X) \rightarrow 0 .
$$

Note also that by arguing by contradiction with the help of $(\star)$ and Theorem 6.2 , it follows from the assumption $(\dagger)$ that for all sufficiently large $k$,

$$
\left(\{0\} \times \mathbb{R}^{n-1}\right) \cap B_{1 / 2} \subset\left(\left\{Z \in \operatorname{spt}\left\|V_{k}\right\|: \Theta\left(\left\|V_{k}\right\|, Z\right) \geq q\right\}\right)_{\tau},
$$

and hence Lemma 7.3 is applicable to the $V_{k}$ 's. Inequality (7.14), Lemma 7.3 (with $\mu=1 / 2$ ) and (7.15) then imply that

$$
\hat{E}_{k}^{-2}\left|F_{k}\right| \leq C \sup |D \zeta| \tau^{1 / 2}
$$

for all sufficiently large $k$, where $C=C(n, q) \in(0, \infty)$.

$$
\begin{aligned}
& \text { Abbreviating } w_{k}=\sum_{j=1}^{q} \frac{\left|D u_{k}^{j}\right|^{2}}{1+\sqrt{1+\left|D u_{k}^{j}\right|^{2}}} D_{2} \zeta-\frac{D_{2} u_{k}^{j}\left(D \zeta \cdot D u_{k}^{j}\right)}{\sqrt{1+\left|D u_{k}^{j}\right|^{2}}} \text {, note that } \\
& \int_{B_{1 / 4} \backslash \Sigma_{k} \cap\left\{x^{2} \leq \tau\right\}}\left|w_{k}\right| \leq C \sup |D \zeta| \int_{\left(\mathbb{R} \times B_{1 / 2}\right) \cap\left\{x^{2} \leq \tau\right\}}\left|\nabla^{V_{k}} x^{1}\right|^{2} d\left\|V_{k}\right\|(X),
\end{aligned}
$$

and by (7.14),

$$
\int_{B_{1 / 4} \cap \Sigma_{k}}\left|w_{k}\right| \leq C \sup |D \zeta| \int_{\left(\mathbb{R} \times B_{1 / 2}\right) \cap\left\{x^{2} \leq \tau\right\}}\left|\nabla^{V_{k}} x^{1}\right|^{2} d\left\|V_{k}\right\|(X)
$$

where $C=C(n)$, so that again by Lemma 7.3 with $\mu=1 / 2$ and (7.15),

$$
\hat{E}_{k}^{-2}\left(\int_{B_{1 / 4} \backslash \Sigma_{k} \cap\left\{x^{2} \leq \tau\right\}}\left|w_{k}\right|+\int_{B_{1 / 4} \cap \Sigma_{k}}\left|w_{k}\right|\right) \leq C \sup |D \zeta| \tau^{1 / 2}
$$

for all sufficiently large $k$, where $C=C(n)$. Finally, by (7.9),

$$
\lim _{k \rightarrow \infty} \hat{E}_{k}^{-2} \int_{B_{1 / 4} \cap\left\{x^{2} \geq \tau\right\}} w_{k}=-\frac{1}{2} \sum_{j=1}^{q} \int_{B_{1 / 4} \cap\left\{x^{2} \geq \tau\right\}}\left|D_{2} v^{j}\right|^{2} D_{2} \zeta
$$

which also uses the fact that $D_{i} v^{j} \equiv 0$ for $i=3, \ldots,(n+1)$ and $j=$ $1,2, \ldots, q$. Dividing (7.12) by $\hat{E}_{k}^{2}$ and first letting $k \rightarrow \infty$ and then letting $\tau \rightarrow 0$ imply, in view of (7.17), (7.18) and (7.19), that

$$
\sum_{j=1}^{q} \int_{B_{1 / 4} \cap\left\{x^{2} \geq 0\right\}}\left|D_{2} v^{j}\right|^{2} D_{2} \zeta=0
$$


for any $\zeta \in C_{c}^{1}\left(B_{1 / 4}\right)$. Since $v^{j}=L_{2}^{j}$ on $\left\{x^{2} \geq 0\right\}$, this contradicts (for any choice of $\zeta \in C_{c}^{1}\left(B_{1 / 4}\right)$ with $\left.\int_{B_{1 / 4} \cap\left\{x^{2} \geq 0\right\}} D_{2} \zeta \neq 0\right)$ our assumption that $L_{2}^{j} \neq L_{2}^{j+1}$ for some $j \in\{1,2, \ldots, q-1\}$.

7.6. Minimum Distance Property for coarse blow-ups: Part III. Let $v$ be as in 7.4.2. The second case of the proof that $\mathcal{B}_{q}$ satisfies property $(\mathcal{B} 7)$ is to rule out the possibility that there are at least two distinct linear functions defining $v$ on either side, i.e. to establish the following:

Lemma 7.5 ([Wic14a], Corollary 14.2]). Let $v=\left(v^{1}, v^{2}, \ldots, v^{q}\right)$ be as in 7.4.2. There exist no indices $i, j \in\{1,2, \ldots, q-1\}$ such that $L_{1}^{i} \neq L_{1}^{i+1}$ and $L_{2}^{j} \neq L_{2}^{j+1}$; consequently (in view of Lemma 7.4), $v^{j}=L$ for some linear function $L$ and each $j=1,2, \ldots, q$, and $\mathcal{B}_{q}$ satisfies property $(\mathcal{B} 7)$.

Remark: Lemma 7.5 is much more subtle than Lemma 7.4. It is false if we drop either the stability hypothesis or the no-classical-singularities hypothesis on the varifolds $V_{k}$ giving rise to $v$, as can be seen by considering in the former case an appropriate sequence of minimal surfaces in the Scherk's family of surfaces and in the latter an appropriate sequence of pairs of transverse planes. For Lemma 7.5, all one can deduce by applying the argument for Lemma 7.4 above is the balancing condition $\sum_{j=1}^{q} m_{j}^{2}=$ $\sum_{j=1}^{q} \ell_{j}^{2}$ where $\mu_{j}=\left|D L_{1}^{j}\right|$ and $\ell_{j}=D L_{2}^{j}, 1 \leq j \leq q$. Indeed, Lemma 7.5 takes substantially more effort to prove than Lemma 7.4, and carrying out its proof takes up more than one third of the length of the paper [Wic14a].

To describe the basic idea of the proof of Lemma 7.5, let $v \in \mathcal{B}_{q}$ and let the linear functions $L_{1}^{j}, L_{2}^{j}: \mathbb{R}^{n} \rightarrow \mathbb{R}, 1 \leq j \leq q$, be as defined in (7.7), and assume, contrary to the assertion of Lemma 7.5, that we have for some $i, j \in\{1,2, \ldots, q\}$,

$$
L_{1}^{i} \neq L_{1}^{i+1} \quad \text { and } \quad L_{2}^{j} \neq L_{2}^{j+1} .
$$

In view of properties $(\mathcal{B} 3)$ and $(\mathcal{B} 5)$, we may assume without loss of generality that

$$
\begin{gathered}
\sum_{j=1}^{q} L_{k}^{j}=0 \quad \text { for } k=1,2, \quad \text { and that } \\
\|v\|_{L^{2}\left(B_{1}\right)}^{2}\left(=\sum_{j=1}^{q}\left\|L_{1}^{j}\right\|_{B_{1} \cap\left\{x^{2} \geq 0\right\}}^{2}+\left\|L_{2}^{j}\right\|_{B_{1} \cap\left\{x^{2}<0\right\}}^{2}\right)=1 .
\end{gathered}
$$

Let $\left\{V_{k}\right\} \subset \mathcal{S}$ be a sequence of varifolds whose coarse blow-up is $v$. The proof of Lemma 7.5 is accomplished by showing that under the hypotheses $(7.20),(7.21),(7.22)$, one can find $k$ sufficiently large such that there exists a point $Z_{k} \in \operatorname{sing} V_{k}$ with the property that a suitable sequence of re-scalings followed by rotations of $V_{k}$ about $Z_{k}$ converges to a cone supported on 
at least four distinct half-hyperplanes meeting along a common boundary, contradicting directly the induction hypothesis $(H 2)$.

It will be convenient to use the following notation for the rest of this discussion where we shall explain in more detail how this contradiction is obtained.

(N1) Let $\mathcal{C}_{q}$ denote the set of hypercones $\mathbf{C}$ of $\mathbb{R}^{n+1}$ of the form $\mathbf{C}=$ $\sum_{j=1}^{q}\left|H_{j}\right|+\left|G_{j}\right|$, where for each $j \in\{1,2, \ldots, q\}, H_{j}$ is the half-hyperplane defined by $H_{j}=\left\{\left(x^{1}, x^{2}, y\right) \in \mathbb{R}^{n+1}: x^{2}<0\right.$ and $\left.x^{1}=\lambda_{j} x^{2}\right\}, G_{j}$ the halfhyperplane defined by $G_{j}=\left\{\left(x^{1}, x^{2}, y\right) \in \mathbb{R}^{n+1}: x^{2}>0\right.$ and $\left.x^{1}=\mu_{j} x^{2}\right\}$, with $\lambda_{j}, \mu_{j}$ constants, $\lambda_{1} \geq \lambda_{2} \geq \ldots \geq \lambda_{q}$ and $\mu_{1} \leq \mu_{2} \leq \ldots \leq \mu_{q}$. We do not assume that the cones in $\mathcal{C}_{q}$ are stationary in $\mathbb{R}^{n+1}$.

(N2) For $V \in \mathcal{S}$ and $\mathbf{C} \in \mathcal{C}_{q}$ define a height excess ("fine excess") $Q_{V}(\mathbf{C})$ of $V$ relative to $\mathbf{C}$ by

$$
\begin{aligned}
& Q_{V}(\mathbf{C})=\left(\int_{\mathbb{R} \times\left(B_{1 / 2} \backslash\left\{\left|x^{2}\right|<1 / 16\right\}\right)} \operatorname{dist}^{2}(X, \operatorname{spt}\|V\|) d\|\mathbf{C}\|(X)\right. \\
&\left.+\int_{\mathbb{R} \times B_{1}} \operatorname{dist}^{2}(X, \operatorname{spt}\|\mathbf{C}\|) d\|V\|(X)\right)^{1 / 2} .
\end{aligned}
$$

Let $\mathbf{C}_{k} \in \mathcal{C}_{q}$ be the cone corresponding to $\hat{E}_{V_{k}} v$, i.e.

$$
\mathbf{C}_{k}=\sum_{j=1}^{q}\left|\operatorname{graph} \hat{E}_{V_{k}} L_{1}^{j}\right|\left\llcorner\left\{x^{2} \geq 0\right\}+\left|\operatorname{graph} \hat{E}_{V_{k}} L_{2}^{j}\right|\left\llcorner\left\{x^{2}<0\right\} .\right.\right.
$$

It is possible to verify with the help of $(\star)$ and Theorem 6.2 that for any given $\epsilon, \gamma \in(0,1)$, after passing to appropriate subsequences of $\left\{V_{k}\right\}$ and $\left\{\mathbf{C}_{k}\right\}$ without changing notation, there exist points $Z_{k} \in \operatorname{spt}\left\|V_{k}\right\|$ with $\Theta\left(\left\|V_{k}\right\|, Z_{k}\right) \geq q$ and $Z_{k} \rightarrow 0$ such that for each $k$, Hypotheses 7.1 below are satisfied with $\widetilde{V}_{k} \equiv \eta_{Z_{k}, 2\left(1-\left|Z_{k}\right|\right) \#} V_{k}$ in place of $V$ and $\mathbf{C}_{k}$ in place of $\mathbf{C}$. (See the proof of [[Wic14a], Corollary 14.2] for details.) Note that $\hat{E}_{\widetilde{V}_{k}} \rightarrow 0$, and that the coarse blow-up of the sequence $\left\{\widetilde{V}_{k}\right\}$ (relative to $\left\{\mathbf{C}_{k}\right\}$ ) is still $v$.

Hypotheses 7.1 (Contradiction Hypotheses).

(1) $V \in \mathcal{S}, \quad \Theta(\|V\|, 0) \geq q, \quad\left(\omega_{n} 2^{n}\right)^{-1}\|V\|\left(B_{2}^{n+1}(0)\right)<q+1 / 2$, $\omega_{n}^{-1}\|V\|\left(\mathbb{R} \times B_{1}\right)<q+1 / 2$.

(2) $\mathbf{C} \in \mathcal{C}_{q}$.

(3) $\hat{E}_{V}^{2} \equiv \int_{\mathbb{R} \times B_{1}}\left|x^{1}\right|^{2} d\|V\|(X)<\epsilon$.

(4) $\{Z: \Theta(\|V\|, Z) \geq q\} \cap\left(\mathbb{R} \times\left(B_{1 / 2} \backslash\left\{\left|x^{2}\right|<1 / 16\right\}\right)\right)=\emptyset$.

(5) $Q_{V}^{2}(\mathbf{C})<\gamma \hat{E}_{V}^{2}$.

One can also check, in view of (7.21) and (7.22), that passing to a subsequence of $\left\{\widetilde{V}_{k}\right\}$ without changing notation, the following hypothesis is satisfied with $M=1$ and with $\widetilde{V}_{k}$ in place of $V$ : 


\section{Hypothesis $(\star)$ :}

$$
\hat{E}_{V}^{2}<\frac{3}{2} M \inf _{\left\{P=\left\{x^{1}=\lambda x^{2}\right\} \in G_{n}: \lambda \in \mathbb{R}\right\}} \int_{\mathbb{R} \times B_{1}} \operatorname{dist}^{2}(X, P) d\|V\|(X) .
$$

Thus, the lemma below can be applied iteratively starting with $V=\widetilde{V}_{k}$ and $\mathbf{C}=\mathbf{C}_{k}$ for suitably large fixed $k$ to produce a sequence of numbers $\sigma_{j} \rightarrow 0$, a sequence of rotations $\Gamma_{j}$ of $\mathbb{R}^{n+1}$ and a cone $\mathbf{W}$ supported on four or more distinct half-hyperplanes meeting along a common axis and with $\Theta(\|\mathbf{W}\|, 0)=q$ such that

$$
\eta_{\sigma_{j} \#} \Gamma_{j \#} \widetilde{V_{k}} \rightarrow \mathbf{W}
$$

as varifolds as $j \rightarrow \infty$, directly contradicting the induction hypothesis $(H 2)$ as desired. In this lemma and subsequently, $M_{0}$ denotes a certain explicit constant $>1$ that depends only on $n$ and $q$.

Lemma 7.6 ([Wic14a], Lemma 13.3). Let $q \geq 2$ be an integer. For $j=1,2, \ldots, 2 q-3$, let $\theta_{j} \in(0,1 / 4)$ be such that $\theta_{1}>8 \theta_{2}>64 \theta_{3}>\ldots>$ $8^{2 q-4} \theta_{2 q-3}$. There exist numbers $\epsilon=\epsilon\left(n, q, \theta_{1}, \theta_{2}, \ldots, \theta_{2 q-3}\right) \in(0,1 / 2)$, $\gamma=\gamma\left(n, q, \theta_{1}, \theta_{2} \ldots, \theta_{2 q-3}\right) \in(0,1 / 2)$ such that if the varifold $V$ and the cone $\mathbf{C}$ satisfy Hypotheses 7.1 and Hypothesis ( $\star$ ) with $M=M_{0}$, and if the induction hypotheses $(H 1),(H 2)$ hold, then there exist an orthogonal rotation $\Gamma$ of $\mathbb{R}^{n+1}$ and a cone $\mathbf{C}^{\prime} \in \mathcal{C}_{q}$ satisfying

(a) $\left|e_{1}-\Gamma\left(e_{1}\right)\right| \leq \kappa Q_{V}(\mathbf{C}), \quad\left|e_{j}-\Gamma\left(e_{j}\right)\right| \leq \kappa \hat{E}_{V}^{-1} Q_{V}(\mathbf{C})$

for each $j=2,3, \ldots,(n+1)$;

(b) $\operatorname{dist}_{\mathcal{H}}^{2}\left(\operatorname{spt}\left\|\mathbf{C}^{\prime}\right\| \cap\left(\mathbb{R} \times B_{1}\right)\right.$, spt $\left.\|\mathbf{C}\| \cap\left(\mathbb{R} \times B_{1}\right)\right) \leq C_{0} Q_{V}^{2}(\mathbf{C}) ;$ and the following for some $j \in\{1,2, \ldots, 2 q-3\}$ :

(c) $\theta_{j}^{-n-2} \int_{\Gamma\left(\mathbb{R} \times\left(B_{\theta_{j} / 2} \backslash\left\{\left|x^{2}\right| \leq \theta_{j} / 16\right\}\right)\right)} \operatorname{dist}^{2}(X$, spt $\|V\|) d\left\|\Gamma_{\#} \mathbf{C}^{\prime}\right\|(X)$

$$
+\theta_{j}^{-n-2} \int_{\Gamma\left(\mathbb{R} \times B_{\theta_{j}}\right)} \operatorname{dist}^{2}\left(X, \operatorname{spt}\left\|\Gamma_{\#} \mathbf{C}^{\prime}\right\|\right) d\|V\|(X) \leq \nu_{j} \theta_{j}^{2} Q_{V}^{2}(\mathbf{C}) ;
$$

(d) $\left(\theta_{j}^{-n-2} \int_{\mathbb{R} \times B_{\theta_{j}}} \operatorname{dist}^{2}(X, P) d\left\|\Gamma_{\#}^{-1} V\right\|(X)\right)^{1 / 2}$

$$
\geq C_{1} \operatorname{dist}_{\mathcal{H}}\left(\operatorname{spt}\|\mathbf{C}\| \cap\left(\mathbb{R} \times B_{1}\right), P \cap\left(\mathbb{R} \times B_{1}\right)\right)-C_{2} Q_{V}(\mathbf{C})
$$

for any $P \in G_{n}$ of the form $P=\left\{x^{1}=\lambda x^{2}\right\}$ for some $\lambda \in(-1,1)$;

(e) $\quad\left\{Z: \Theta\left(\left\|\Gamma_{\#}^{-1} V\right\|, Z\right) \geq q\right\} \cap\left(\mathbb{R} \times\left(B_{\theta_{j} / 2} \cap\left\{\left|x^{2}\right|<\theta_{j} / 16\right\}\right)\right)=\emptyset$;

(f) $\left(\omega_{n} \theta_{j}^{n}\right)^{-1}\left\|\Gamma_{\#}^{-1} V\right\|\left(\mathbb{R} \times B_{\theta_{j}}\right)<q+1 / 2$.

Here $C_{1}=C_{1}(n)$; the constants $\kappa, C_{0}, C_{2} \in(0, \infty)$ depend only on $n$ in case $q=2$ and only on $n, q$ and $\theta_{1}, \theta_{2}, \ldots, \theta_{2 q-4}$ in case $q \geq 3$; $\nu_{1}=\nu_{1}(n, q) ;$ and, in case $q \geq 3, \nu_{j}=\nu_{j}\left(n, q, \theta_{1}, \ldots, \theta_{j-1}\right)$ for each 
$j=2,3, \ldots, 2 q-3$. In particular, $\nu_{j}$ is independent of $\theta_{j}, \theta_{j+1}, \ldots, \theta_{2 q-3}$ for each $j=1,2, \ldots, 2 q-3$.

We shall give a brief description of the proof of this lemma after making the following remarks.

Remarks: (1) An interesting feature of Lemma 7.6 is that its conclusions guarantee improvement of the fine excess $Q_{V}(\mathbf{C})$ and the validity of the starting hypotheses at one of finitely many possible fixed smaller scales $\theta_{1}, \theta_{2}, \ldots, \theta_{2 q-4}$, unlike in similar excess improvement lemmas found in earlier work such as that of De Giorgi [DG61], Allard [All72], SchoenSimon [SS81] and Simon [Sim93] where the conclusions always involve a single fixed smaller scale.

Although in the present application of Lemma 7.6 we are not interested in any $C^{1, \alpha}$ regularity conclusions (as it suffices at this stage of the argument merely to produce a single singular cone $\mathbf{W} \in \mathcal{C}_{q}$ to which a suitable sequence of varifolds in $\mathcal{S}$ converges - as in (7.23) - in order to obtain a contradiction to the induction hypothesis (H2) ), generally speaking, allowing multiple scales in an excess decay lemma in this manner would actually still suffice for such regularity conclusions. That this is so will be important later in carrying out the induction step for the Minimum Distance Theorem where what we shall have at our disposal is a similar lemma allowing multiple scales and yet what we need is to make an appropriate $C^{1, \alpha}$ decay conclusion in order to use (finally) the no-classical-singularities hypothesis non-inductively. See Section 8 below.

Allowing multiple scales is a natural trick employed in [Wic14a] to overcome certain issues arising from the presence of higher multiplicity. See the remark following Lemma 7.7 below.

(2) In view of the fact that for each $j \in\{1,2, \ldots, 2 q-3\}$ the constant $\nu_{j}$ in the lemma is independent of the scales $\theta_{j}, \theta_{j+1}, \ldots, \theta_{2 q-3}$, it is possible to choose the scales $\theta_{1}, \theta_{2}, \ldots, \theta_{2 q-3}$ depending only on $n$ and $q$ such that $\nu_{j} \theta_{j}^{2}<4^{-1}$ for each $j=1,2, \ldots, 2 q-3$. With such a choice and under the hypotheses of the lemma, Part (c) of the conclusion of the lemma says that the fine-excess of suitably rotated and rescaled $V$ relative to a new cone $\in \mathcal{C}_{q}$ improves by a factor $4^{-1}$ at each iteration of the lemma. Parts (b), (d), (e), (f) of the conclusion imply that the original hypotheses (i.e. Hypothesis 7.1 and Hypothesis $(\star))$ are satisfied at the new scale, so the lemma can be iterated indefinitely to produce scales $\sigma_{j} \rightarrow 0$, rotations $\Gamma_{j}$ and a non-planar cone $\mathbf{W} \in \mathcal{C}_{q}$ such that the desired conclusion (7.23) holds.

7.6.1. Proof of Lemma 7.6. The first step in proving Lemma 7.6 is to reduce it to a result (Lemma 7.7 below) in the same spirit but with stronger hypotheses and stronger conclusions; specifically, to show that the conclusions of Lemma 7.6 hold at an arbitrary single scale $\theta \in(0,1 / 4)$ (instead of the finite set $\left\{\theta_{1}, \theta_{2}, \ldots, \theta_{2 q-3}\right\}$ of scales allowed in Lemma 7.6) provided we make one additional hypothesis. To describe this hypothesis, it is convenient to use the following notation: 
(N3) For $p \in\{2,3, \ldots, 2 q\}$, let $\mathcal{C}_{q}(p)$ denote the set of hypercones $\mathbf{C}=$ $\sum_{j=1}^{q}\left|H_{j}\right|+\left|G_{j}\right| \in \mathcal{C}_{q}$ as defined in (N1) above such that the number of distinct half-hyperplanes in the set

$$
\left\{H_{1}, \ldots, H_{q}, G_{1}, \ldots, G_{q}\right\}
$$

is $p$. Then, of course, $\mathcal{C}_{q}=\cup_{p=2}^{2 q} \mathcal{C}_{q}(p)$.

(N4) For $q \geq 2$ and $p \in\{4, \ldots, 2 q\}$, let

$$
Q_{V}^{\star}(p)=\inf _{\mathbf{C} \in \cup_{k=4}^{p} \mathcal{C}_{q}(k)} Q_{V}(\mathbf{C})
$$

where $Q_{V}(\mathbf{C})$ is as defined in (N2) above.

With this notation, consider for small $\beta \in(0,1)$ to be chosen depending only on $n$ and $q$ and for $V \in \mathcal{S}$ the following:

Hypothesis $(\star \star)$ : Either

(i) $\mathbf{C} \in \mathcal{C}_{q}(4)$ or

(ii) $q \geq 3, \mathbf{C} \in \mathcal{C}_{q}(p)$ for some $p \in\{5, \ldots, 2 q\}, Q_{V}^{2}(\mathbf{C})<$

$$
\beta\left(Q_{V}^{\star}(p-1)\right)^{2} \text {. }
$$

The idea is that under Hypotheses 7.1 and Hypothesis $(\star \star)$ for suitably small $\epsilon, \gamma$ and $\beta$, the sheets of $V$ away from a small tubular neighborhood $T$ of the singular axis of $\mathbf{C}$ organize themselves as sets of smooth embedded ordered graphs over the distinct half-hyperplanes making up spt $\|\mathbf{C}\|$, with the absolute value of the height function defining the graphs equal to the distance to spt $\|\mathbf{C}\|$. (See conclusion (a) of Lemma 7.8 below). This prevents in a strong way the possibility of concentration of the fine excess $Q_{V}(\mathbf{C})$ in any small region outside $T$.

With Hypothesis $(\star \star)$ in place in addition to the other hypotheses as in Lemma 7.6, the claim now is the following:

Lemma 7.7 ([Wic14a], Lemma 13.1). For any given $\theta \in(0,1 / 4)$, there exists $\epsilon=\epsilon(n, q, \theta) \in(0,1 / 2), \gamma=\gamma(n, q, \theta) \in(0,1 / 2), \beta=\beta(n, q, \theta) \epsilon$ $(0,1 / 2)$ such that if $V \in \mathcal{S}, \mathbf{C} \in \mathcal{C}_{q}$ satisfy Hypotheses 7.1, Hypothesis ( $\star$ ) with $M=M_{0}^{2}$ and Hypothesis ( $\left.\star \star\right)$, and if the induction hypotheses (H1), $(H 2)$ hold, then there exist an orthogonal rotation $\Gamma$ of $\mathbb{R}^{n+1}$ and a cone $\mathbf{C}^{\prime} \in \mathcal{C}_{q}$ such that the conclusions of Lemma 7.6 hold with $\theta$ in place of $\theta_{j}$, a fixed constant $\nu=\nu(n, q)$ in place of $\nu_{j}$, and with $C_{1}=C_{1}(n)$ and $\kappa, C_{0}$, $C_{2}$ depending only on $n, q$ and $\theta$.

Once Lemma 7.7 is in place it is not difficult to deduce Lemma 7.6; that is to say to remove Hypothesis $(\star \star)$ from Lemma 7.7, provided we do not insist that the conclusions hold at a single smaller scale (unless $q=2$ ) and instead allow ourselves the freedom of a fixed set of finitely many (in fact $2 q-3)$ scales $\theta_{1}, \theta_{2}, \ldots, \theta_{2 q-3}$ at one of which the conclusions are required to hold. This is done by inducting on the unique number $p \in\{4,5, \ldots, 2 q\}$ for which $\mathbf{C} \in \mathcal{C}_{q}(p)$. See the proof of Lemmas 13.2 and 13.1 of [Wic14a] for details. 
7.6.2. Proof of Lemma 7.7. We end this section with a very brief account of the proof of Lemma 7.7. The method used-the so called blow-up method - is inspired by the pioneering work of Simon ([Sim93]) where this method was first used to prove asymptotic decay results near non-isolated singularities for minimal submanifolds in certain multiplicity 1 classes (see Section 2 above for the definition of multiplicity 1 class).

The main a priori estimates needed for the blow up argument in the present context are collected in the lemma below.

Lemma 7.8 ([Wic14a], Theorem 10.1, Corollary 10.2 and Lemma 10.8). Let $q$ be an integer $\geq 2, \tau \in(0,1 / 8), \delta \in(0,1 / 8)$ and $\mu \in(0,1)$. For each $\rho \in(0,1 / 4]$, there exist numbers $\epsilon_{0}=\epsilon_{0}(n, q, \delta, \tau, \rho) \in(0,1)$, $\gamma_{0}=\gamma_{0}(n, q, \delta, \tau, \rho) \in(0,1)$ and $\beta_{0}=\beta_{0}(n, q, \tau, \rho) \in(0,1)$ such that the following is true: Let $V \in \mathcal{S}, \mathbf{C} \in \mathcal{C}_{q}$ satisfy Hypotheses 7.1, Hypothesis ( $\star$ ) and Hypothesis ( $\star \star)$ with $M=M_{0}^{3}$ and $\epsilon_{0}, \gamma_{0}, \beta_{0}$ in place of $\epsilon, \gamma, \beta$ respectively. Suppose also that the induction hypotheses (H1), (H2) hold. Write $\mathbf{C}=\sum_{j=1}^{q}\left|H_{j}\right|+\left|G_{j}\right|$ where for each $j \in\{1,2, \ldots, q\}, H_{j}$ is the halfspace defined by $H_{j}=\left\{\left(x^{1}, x^{2}, y\right) \in \mathbb{R}^{n+1}: x^{2}<0\right.$ and $\left.x^{1}=\lambda_{j} x^{2}\right\}, G_{j}$ the half-space defined by $G_{j}=\left\{\left(x^{1}, x^{2}, y\right) \in \mathbb{R}^{n+1}: x^{2}>0\right.$ and $\left.x^{1}=\mu_{j} x^{2}\right\}$, with $\lambda_{j}, \mu_{j}$ constants, $\lambda_{1} \geq \lambda_{2} \geq \ldots \geq \lambda_{q}$ and $\mu_{1} \leq \mu_{2} \leq \ldots \leq \mu_{q}$; for $\left(x^{2}, y\right) \in \mathbb{R}^{n}$ and $j=1,2, \ldots, q$, define $h_{j}\left(x^{2}, y\right)=\lambda_{j} x^{2}$ and $g_{j}\left(x^{2}, y\right)=$ $\mu_{j} x^{2}$. Then, after possibly replacing $\mathbf{C}$ with another cone $\mathbf{C}^{\prime} \in \mathcal{C}_{q}$ with spt $\left\|\mathbf{C}^{\prime}\right\|=\operatorname{spt}\|\mathbf{C}\|$ and relabelling $\mathbf{C}^{\prime}$ as $\mathbf{C}$, the following must hold for each $Z=\left(\zeta^{1}, \zeta^{2}, \eta\right) \in \operatorname{spt}\|V\| \cap\left(\mathbb{R} \times B_{3 / 8}\right)$ with $\Theta(\|V\|, Z) \geq q$ :

(a) $V\left\llcorner\left(\mathbb{R} \times\left(B_{3 / 4} \backslash\left\{\left|x^{2}\right|<\tau\right\}\right)\right)\right.$

$$
=\sum_{j=1}^{q}\left|\operatorname{graph}\left(h_{j}+u_{j}\right)\right|+\left|\operatorname{graph}\left(g_{j}+w_{j}\right)\right|
$$

where, for each $j=1,2, \ldots, q$,

$u_{j} \in C^{2}\left(B_{3 / 4} \cap\left\{x^{2}<-\tau\right\}\right) ; w_{j} \in C^{2}\left(B_{3 / 4} \cap\left\{x^{2}>\tau\right\}\right) ;$

$h_{j}+u_{j}$ and $g_{j}+w_{j}$ solve the minimal surface equation on their domains;

$h_{1}+u_{1} \leq h_{2}+u_{2} \leq \ldots \leq h_{q}+u_{q} ; g_{1}+w_{1} \leq g_{2}+w_{2} \leq \ldots \leq g_{q}+w_{q} ;$

$\operatorname{dist}\left(\left(h_{j}\left(x^{2}, y\right)+u_{j}\left(x^{2}, y\right), x^{2}, y\right)\right.$, spt $\left.\|\mathbf{C}\|\right)=\left(1+\lambda_{j}^{2}\right)^{-1 / 2}\left|u_{j}\left(x^{2}, y\right)\right|$

for $\left(x^{2}, y\right) \in B_{3 / 4} \cap\left\{x^{2}<-\tau\right\}$;

$\operatorname{dist}\left(\left(g_{j}\left(x^{2}, y\right)+w_{j}\left(x^{2}, y\right), x^{2}, y\right)\right.$, spt $\left.\|\mathbf{C}\|\right)=\left(1+\mu_{j}^{2}\right)^{-1 / 2}\left|w_{j}\left(x^{2}, y\right)\right|$

for $\left(x^{2}, y\right) \in B_{3 / 4} \cap\left\{x^{2}>\tau\right\}$.

(b) $\left|\zeta^{1}\right|^{2}+\hat{E}_{V}^{2}\left|\zeta^{2}\right|^{2} \leq C \int_{\mathbb{R} \times B_{1}} \operatorname{dist}^{2}(X, \operatorname{spt}\|\mathbf{C}\|) d\|V\|(X) ;$ 
(c) $\int_{B_{5 \rho / 8}^{n+1}(Z)} \frac{\operatorname{dist}^{2}\left(X, \text { spt }\left\|T_{Z \#} \mathbf{C}\right\|\right)}{|X-Z|^{n+2-\mu}} d\|V\|(X)$

$$
\leq \widetilde{C} \rho^{-n-2+\mu} \int_{\mathbb{R} \times B_{\rho}\left(\zeta^{2}, \eta\right)} \operatorname{dist}^{2}\left(X, \operatorname{spt}\left\|T_{Z \#} \mathbf{C}\right\|\right) d\|V\|(X) .
$$

(d) $B_{\delta}^{n+1}(0, y) \cap\{Z: \Theta(\|V\|, Z) \geq q\} \neq \emptyset$

for each point $(0, y) \in\{0\} \times \mathbb{R}^{n-1} \cap B_{1 / 2}$.

(e) $\int_{B_{1 / 2}^{n+1}(0) \cap\left\{\left|\left(x^{1}, x^{2}\right)\right|<\sigma\right\}} \operatorname{dist}^{2}(X$, spt $\|\mathbf{C}\|) d\|V\|(X)$

$$
\leq C_{1} \sigma^{1-\mu} \int_{\mathbb{R} \times B_{1}} \operatorname{dist}^{2}(X, \operatorname{spt}\|\mathbf{C}\|) d\|V\|(X) \quad \text { for each } \sigma \in[\delta, 1 / 4) .
$$

Here $T_{Z}: \mathbb{R}^{n+1} \rightarrow \mathbb{R}^{n+1}$ is the translation $X \mapsto X+Z ; C=C(n, q) \in$ $(0, \infty), \widetilde{C}=\widetilde{C}(n, q, \mu) \in(0, \infty)$ and $C_{1}=C_{1}(n, q, \mu) \in(0, \infty)$ (In particular, $C, \widetilde{C}, C_{1}$ do not depend on $\rho$ or $\tau$.)

In Simon's work [Sim93], the analogs of the conclusions (a), (b), (c) and (e) are established, under a different set of assumptions, for stationary $n$-varifolds $V$ of arbitrary dimension and codimension. In particular, neither the stability condition nor the non-existence of classical singularities is assumed therein, but crucially, $V$ is assumed to belong to a multiplicity 1 class $\mathcal{V}_{1}$ and also $V$ is assumed to satisfy conclusion (d) (which is crucial for conclusion (e)). Moreover, in place of Hypotheses 7.1(3), 7.1(5), Hypothesis $(\star)$ and Hypotheses $(\star \star)$, it is assumed in [Sim93] that the cone $\mathbf{C}$ and the varifold $V$ are close (as varifolds) to some fixed multiplicity 1 stationary singular cone $\mathbf{C}_{0} \in \mathcal{V}_{1}$ with $\operatorname{dim} \mathcal{S}\left(\mathbf{C}_{0}\right)=k_{\mathcal{V}_{1}}$, with $k_{\mathcal{V}_{1}}$ allowed to take any value $\in\{0,1,2, \ldots, n-1\}$ (but in case $k_{\mathcal{V}_{1}}<n-2$, the cross-section of $\mathbf{C}_{0}$ is assumed to satisfy a certain integrability condition on the homogeneous degree 1 Jacobi fields over the cross-section). Simon's proof of these estimates uses Allard's regularity theorem (Theorem 1.2) in a crucial way.

The key difference in [Wic14a] that gives rise to substantial additional technical difficulties in the proof of the above estimates is that the $n$ varifold $V \in \mathcal{S}$ is allowed to have higher multiplicity on a set of positive $n$-dimensional measure. Although ultimately for $\mathcal{S}$ an "Allard type" theorem does hold, namely the Sheeting Theorem (Theorem 6.1), Lemma 7.8 is needed during the course of the proof of the Sheeting Theorem! At the stage at which Lemma 7.8 is needed, one has the inductive hypotheses $(H 1),(H 2)$, with $(H 1)$ providing a sheeting theorem but only subject to the restriction that the density ratio of $V$ is $\leq q-1 / 2$.

For conclusion (a) of the lemma (which is a regularity statement for $V$ in a region a fixed distance away from the singular axis of the cone C), it turns out that the argument is fairly direct based on the induction hypotheses $(H 1),(H 2)$. For the other conclusions however (which require information on the part of $V$ very near the axis of $\mathbf{C}$ ), a considerable amount 
of delicate additional technical arguments are needed to implement Simon's basic strategy. See [[Wic14a], Section 10] for details.

With the help of Lemma 7.8, the blow up argument needed to establish Lemma 7.7 proceeds as follows:

Let $\left\{\epsilon_{k}\right\},\left\{\gamma_{k}\right\}$ and $\left\{\beta_{k}\right\}$ be sequences of positive numbers such that

$$
\epsilon_{k}, \gamma_{k}, \beta_{k} \rightarrow 0 \text {. }
$$

Consider sequences of varifolds $V_{k} \in \mathcal{S}$ and cones $\mathbf{C}_{k} \in \mathcal{C}_{q}$ such that, for each $k=1,2, \ldots$, with $V_{k}, \mathbf{C}_{k}$ in place of $V, \mathbf{C}$ respectively, Hypotheses 7.1 hold with $\epsilon_{k}, \gamma_{k}$ in place of $\epsilon, \gamma$; Hypothesis $(\star)$ holds with $M=M_{0}^{3}$ and Hypothesis $(\star \star)$ holds with $\beta_{k}$ in place of $\beta$.

Let $\left\{\delta_{k}\right\},\left\{\tau_{k}\right\}$ be sequences of decreasing positive numbers converging to 0 , and let

$$
E_{k}=\left(\int_{\mathbb{R} \times B_{1}} \operatorname{dist}^{2}\left(X, \operatorname{spt}\left\|\mathbf{C}_{k}\right\|\right) d\left\|V_{k}\right\|(X)\right)^{1 / 2} .
$$

By passing to appropriate subsequences of $\left\{V_{k}\right\},\left\{\mathbf{C}_{k}\right\}$, and possibly replacing $\mathbf{C}_{k}$ with a cone $\mathbf{C}_{k}^{\prime} \in \mathcal{C}_{q}$ with spt $\left\|\mathbf{C}_{k}^{\prime}\right\|=\operatorname{spt}\left\|\mathbf{C}_{k}\right\|$ without changing notation, we obtain functions $u_{j}^{(k)} \in C^{2}\left(B_{3 / 4} \cap\left\{x^{2}<-\tau_{k}\right\}\right)$ and $w_{j}^{(k)} \in C^{2}\left(B_{3 / 4} \cap\left\{x^{2}>\tau_{k}\right\}\right), 1 \leq j \leq q$, satisfying the conclusions of Lemma 7.8 with $V_{k}$ in place of $V, \mathbf{C}_{k}$ in place of $\mathbf{C}, u_{j}^{(k)}, w_{j}^{(k)}$ in place of $u_{j}, w_{j}$ and with $\delta_{k}, \tau_{k}$ in place of $\delta, \tau$ respectively. By Lemma 7.8(a) and elliptic estimates, there exist harmonic functions $\varphi_{j} \in C^{2}\left(B_{3 / 4} \cap\left\{x^{2}<0\right\}\right)$ and $\psi_{j} \in C^{2}\left(B_{3 / 4} \cap\left\{x^{2}>0\right\}\right), 1 \leq j \leq q$ such that

$$
E_{k}^{-1} u_{j}^{(k)} \rightarrow \varphi_{j} \quad \text { and } \quad E_{k}^{-1} w_{j}^{(k)} \rightarrow \psi_{j}
$$

where the convergence is locally in $C^{2}$ in the respective domains. It also follows from Lemma 7.8(e) that $\varphi_{j} \in L^{2}\left(B_{1 / 2} \cap\left\{x^{2}<0\right\}\right)$ and $\psi_{j} \in$ $L^{2}\left(B_{1 / 2} \cap\left\{x^{2}>0\right\}\right)$, and that the convergence above is also in $L^{2}\left(B_{1 / 2} \cap\right.$ $\left.\left\{x^{2}<0\right\}\right)$ and $L^{2}\left(B_{1 / 2} \cap\left\{x^{2}>0\right\}\right)$ respectively. Write $\varphi=\left(\varphi_{1}, \varphi_{2}, \ldots, \varphi_{q}\right)$ and $\psi=\left(\psi_{1}, \psi_{2}, \ldots, \psi_{q}\right)$

Definition: Let $\mathcal{B}_{q}^{F}$ be the (smaller) class of pairs of functions $(\varphi, \psi)$ (the fine blow-ups) arising as above corresponding to sequences $V_{k}, \mathbf{C}_{k}$ satisfying all of the hypotheses required as above but with $M=M_{0}^{2}$ in Hypothesis $(\star)$.

What is required next in order to prove Lemma 7.7 is sufficiently strong uniform regularity estimates for the functions $\varphi, \psi$ with $(\varphi, \psi) \in \mathcal{B}_{q}^{F}$ in fixed smaller regions in their domains, e.g. in $B_{1 / 4} \cap\left\{x^{2}<0\right\}$ and $B_{1 / 4} \cap\left\{x^{2}>0\right\}$ respectively, necessarily up to the boundary $B_{1 / 4} \cap\left\{x^{2}=0\right\}$. For instance a uniform $C^{1, \alpha}$ estimate up to the boundary $B_{1 / 4} \cap\left\{x^{2}=0\right\}$ for some fixed $\alpha \in(0,1)$ suffices.

The first step in obtaining such regularly estimates is the following uniform continuity estimate [[Wic14a], Lemma 12.1], which is essentially a consequence of Lemma 7.8(c): 
There exist two functions $\kappa_{1}, \kappa_{2}: B_{5 / 16} \cap\left\{x^{2}=0\right\} \rightarrow \mathbb{R}$ with

$$
\left|\kappa_{1}(Y)\right|,\left|\kappa_{2}(Y)\right| \leq C \quad \text { for } \quad Y \in B_{5 / 16} \cap\left\{x^{2}=0\right\}
$$

and numbers $\ell_{j}, m_{j}(1 \leq j \leq q)$ with $\ell_{1} \geq \ldots \geq \ell_{q}, m_{1} \leq \ldots \leq m_{q}$,

$$
\begin{gathered}
c \leq \max \left\{\left|\ell_{1}\right|,\left|\ell_{q}\right|\right\} \leq c_{1}, \quad c \leq \max \left\{\left|m_{1}\right|,\left|m_{q}\right|\right\} \leq c_{1} \quad \text { and } \\
\min \left\{\left|\ell_{1}-\ell_{q}\right|,\left|m_{1}-m_{q}\right|\right\} \geq 2 c
\end{gathered}
$$

where $C=C(n, q), c=c(n, q), c_{1}=c_{1}(n, q)$, such that

$$
\begin{aligned}
& \sum_{j=1}^{q} \int_{B_{\rho / 2}(Y) \cap\left\{x^{2}<0\right\}} \frac{\left|\varphi_{j}(x)-\left(\kappa_{1}(Y)-\ell_{j} \kappa_{2}(Y)\right)\right|^{2}}{|x-Y|^{n+3 / 2}} d x \\
& +\sum_{j=1}^{q} \int_{B_{\rho / 2}(Y) \cap\left\{x^{2}>0\right\}} \frac{\left|\psi_{j}(x)-\left(\kappa_{1}(Y)-m_{j} \kappa_{2}(Y)\right)\right|^{2}}{|x-Y|^{n+3 / 2}} d x \\
& \leq C_{1} \rho^{-n-3 / 2} \sum_{j=1}^{q} \int_{B_{\rho}(Y) \cap\left\{x^{2}<0\right\}}\left|\varphi_{j}-\left(\kappa_{1}(Y)-\ell_{j} \kappa_{2}(Y)\right)\right|^{2} \\
& +C_{1} \rho^{-n-3 / 2} \sum_{j=1}^{q} \int_{B_{\rho}(Y) \cap\left\{x^{2}>0\right\}}\left|\psi_{j}-\left(\kappa_{1}(Y)-m_{j} \kappa_{2}(Y)\right)\right|^{2}
\end{aligned}
$$

where $C_{1}=C_{1}(n, q) \in(0, \infty)$.

This estimate implies (see the proof of [Wic14a], Lemma 12.1) that $\varphi, \psi$ are respectively uniformly Hölder continuous in $B_{5 / 16} \cap\left\{x^{2} \leq 0\right\}$, $B_{5 / 16} \cap\left\{x^{2} \geq 0\right\}$ with a fixed Hölder exponent $\beta=\beta(n, q) \in(0,1)$. In particular the functions $\varphi(Y) \equiv\left(\kappa_{1}(Y)-\ell_{1} \kappa_{2}(Y), \ldots, \kappa_{1}(Y)-\ell_{q} \kappa_{2}(Y)\right)$, $\psi(Y) \equiv\left(\kappa_{1}(Y)-m_{1} \kappa_{2}(Y), \ldots, \kappa_{1}(Y)-m_{q} \kappa_{2}(Y)\right)$ are respectively the continuous boundary values of $\varphi, \psi$ on $B_{5 / 16} \cap\left\{x^{2}=0\right\}$.

In order to improve regularity of $(\varphi, \psi)$, it then suffices to show that the boundary values $\varphi(Y), \psi(Y)$ as functions of $Y \in B_{1 / 4} \cap\left\{x^{2}=0\right\}$ are more regular. This is achieved by employing two separate first variation arguments based on the fact that, since the varifolds $V_{k}$ giving rise to the blow-up $(\varphi, \psi)$ are stationary, the restrictions of the ambient coordinate functions $\pi_{1}(X)=x^{1}$ and $\pi_{2}(X)=x^{2}$ to each $V_{k}$ are weakly harmonic on $V_{k}$. Starting with this, it can be shown (see [[Wic14a], Lemma 12.2]) that the two linear combinations of $\kappa_{1}, \kappa_{2}$ defined by

$$
\begin{gathered}
\Phi(Y)=2 q \kappa_{1}(Y)-\left(\sum_{j=1}^{q}\left(\ell_{j}+m_{j}\right)\right) \kappa_{2}(Y) \text { and } \\
\Psi(Y)=\left(\sum_{j=1}^{q}\left(\ell_{j}+m_{j}\right)\right) \kappa_{1}(Y)-\left(\sum_{j=1}^{q}\left(\ell_{j}^{2}+m_{j}^{2}\right)\right) \kappa_{2}(Y)
\end{gathered}
$$

for $Y \in B_{9 / 32} \cap\left\{x^{2}=0\right\}$ are smooth functions satisfying 


$$
\begin{aligned}
& \|\Phi\|_{C^{3}\left(B_{9 / 32} \cap\left\{x^{2}=0\right\}\right)}+\|\Psi\|_{C^{3}\left(B_{9 / 32} \cap\left\{x^{2}=0\right\}\right)} \\
& \leq C\left(\int_{B_{1 / 2} \cap\left\{x^{2} \leq 0\right\}}|\varphi|^{2}+\int_{B_{1 / 2} \cap\left\{x^{2} \geq 0\right\}}|\psi|^{2}\right)^{1 / 2}
\end{aligned}
$$

where $C=C(n, q) \in(0, \infty)$. Since the Jacobian $J$ associated with the above linear system for $\kappa_{1}, \kappa_{2}$ is given by

$$
\begin{aligned}
J=2 q \sum_{j=1}^{q}\left(\ell_{j}^{2}\right. & \left.+m_{j}^{2}\right)-\left(\sum_{j=1}^{q}\left(\ell_{j}+m_{j}\right)\right)^{2} \\
& =\frac{1}{2} \sum_{i=1}^{q} \sum_{j=1}^{q}\left(\left(m_{i}-m_{j}\right)^{2}+\left(\ell_{i}-\ell_{j}\right)^{2}+2\left(\ell_{i}-m_{j}\right)^{2}\right),
\end{aligned}
$$

$J$ satisfies, by (7.24), $\widetilde{C} \geq J \geq C>0$ for constants $\widetilde{C}=\widetilde{C}(n, q)$, $C=C(n, q)$. It follows that $\kappa_{j}, j=1,2$, and hence $\left.\varphi\right|_{B_{9 / 32} \cap\left\{x^{2}=0\right\}}$ and $\left.\psi\right|_{B_{9 / 32} \cap\left\{x^{2}=0\right\}}$, are $C^{3}$ on $B_{9 / 32} \cap\left\{x^{2}=0\right\}$, and moreover

$$
\begin{aligned}
\|\varphi\|_{C^{3}\left(B_{9 / 32} \cap\left\{x^{2}=0\right\}\right)}+\|\psi\|_{C^{3}\left(B_{9 / 32} \cap\left\{x^{2}=0\right\}\right)} & \\
\leq C & \left(\int_{B_{1 / 2} \cap\left\{x^{2} \leq 0\right\}}|\varphi|^{2}+\int_{B_{1 / 2} \cap\left\{x^{2} \geq 0\right\}}|\psi|^{2}\right)^{1 / 2}
\end{aligned}
$$

for a constant $C=C(n, q)$. By standard boundary regularity theory for harmonic functions, this leads to the desired improved regularity for $(\varphi, \psi)$, specifically to the conclusion that $\varphi \in C^{2}\left(B_{1 / 4} \cap\left\{x^{2} \leq 0\right\}\right), \quad \psi \in$ $C^{2}\left(B_{1 / 4} \cap\left\{x^{2} \geq 0\right\}\right)$ with

$$
\begin{aligned}
& \|\varphi\|_{C^{2}\left(B_{1 / 4} \cap\left\{x^{2} \leq 0\right\}\right)}+\|\psi\|_{C^{2}\left(B_{1 / 4} \cap\left\{x^{2} \geq 0\right\}\right)} \\
& \leq C\left(\int_{B_{1 / 2} \cap\left\{x^{2}<0\right\}}|\varphi|^{2}+\int_{B_{1 / 2} \cap\left\{x^{2}>0\right\}}|\psi|^{2}\right)^{1 / 2}
\end{aligned}
$$

where $C=C(n, q)$. Lemma 7.7 follows from this estimate in a fairly standard way.

7.7. Sketch of the proof of Theorem 6.1 subject to the induction hypotheses. Once it is established that $\mathcal{B}_{q}$ is a proper blow up class (as defined in Section 7.1), it follows from Lemma 7.1 that each $v \in \mathcal{B}_{q}$ is given by a set of $q$ (classical) harmonic functions on $B_{1}$. Moreover, given $v \in \mathcal{B}_{q}$, a sequence of varifolds $\left\{V_{k}\right\} \subset \mathcal{S}$ giving rise to $v$ and any $\sigma \in(0,1)$, we have the dichotomy that either $\Theta\left(\left\|V_{k}\right\|, Z\right)<q$ for all $k$ sufficiently large and $Z \in \mathbb{R} \times B_{\sigma}(0)$, or (by Lemma 7.1 and the argument, described in Section 7.3 , establishing property $\left(\mathcal{B}_{4}\right)$ for $\mathcal{B}_{q}$ ) that $v^{1}=v^{2}=\ldots=v^{q}$ on $B_{1}$. 
Given this fact, it is fairly straightforward to establish, with the help of $(\star)$ of Section 7.3 and Theorem 6.2, the following lemma:

LEMma 7.9. Let $q$ be an integer $\geq 2, \theta \in(0,1 / 4)$ and suppose that the induction hypotheses (H1), (H2) hold. There exists a number $\beta_{0}=$ $\beta_{0}(n, q, \theta) \in(0,1 / 2)$ such that if $V \in \mathcal{S},\left(\omega_{n} 2^{n}\right)^{-1}\|V\|\left(B_{2}^{n+1}(0)\right)<q+1 / 2$, $q-1 / 2 \leq\left(\omega_{n}\right)^{-1}\|V\|\left(B_{1} \times \mathbb{R}\right)<q+1 / 2$, and

$$
\int_{\mathbb{R} \times B_{1}} \operatorname{dist}^{2}(X, P) d\|V\|(X)<\beta_{0}
$$

for some affine hyperplane $P$ of $\mathbb{R}^{n+1}$ with $\operatorname{dist}_{\mathcal{H}}^{2}\left(P \cap\left(B_{1} \times \mathbb{R}\right), B_{1} \times\{0\}\right)<$ $\beta_{0}$, then the following hold:

(a) Either $V\left\llcorner\left(B_{1 / 2} \times \mathbb{R}\right)=\sum_{j=1}^{q} \mid\right.$ graph $u_{j} \mid$ where $u_{j} \in C^{2}\left(B_{1 / 2} ; \mathbb{R}\right)$ for $j=1,2, \ldots, q$ with $u_{1} \leq u_{2} \leq \ldots \leq u_{q}$ on $B_{1 / 2}, u_{j_{0}}<u_{j_{0}+1}$ on $B_{1 / 2}$ for some $j_{0} \in\{1,2, \ldots, q-1\}$, and for each $j \in\{1,2, \ldots, q\}$,

$$
\begin{aligned}
& \sup _{B_{1 / 2}}\left|u_{j}-p\right|^{2}+\left|D u_{j}-D p\right|^{2}+\left|D^{2} u_{j}\right|^{2} \\
& \leq C \int_{\mathbb{R} \times B_{1}} \operatorname{dist}^{2}(X, P) d\|V\|(X)
\end{aligned}
$$

where $C=C(n, q) \in(0, \infty)$ and $p: \mathbb{R}^{n} \rightarrow \mathbb{R}$ is the affine function such that graph $p=P$; or, there exists an affine hyperplane $P^{\prime}$ with

$$
\begin{aligned}
& \operatorname{dist}_{\mathcal{H}}^{2}\left(P^{\prime} \cap\left(\mathbb{R} \times B_{1}\right), P\right.\left.\cap\left(\mathbb{R} \times B_{1}\right)\right) \\
& \leq C_{1} \int_{\mathbb{R} \times B_{1}} \operatorname{dist}^{2}(X, P) d\|V\|(X) \text { and } \\
& \theta^{-n-2} \int_{\mathbb{R} \times B_{\theta}} \operatorname{dist}^{2}\left(X, P^{\prime}\right) d\|V\|(X) \\
& \leq C_{2} \theta^{2} \int_{\mathbb{R} \times B_{1}} \operatorname{dist}^{2}(X, P) d\|V\|(X)
\end{aligned}
$$

where $C_{1}=C_{1}(n, q) \in(0, \infty)$ and $C_{2}=C_{2}(n, q) \in(0, \infty)$.

(b) $\left(\omega_{n}(2 \theta)^{n}\right)^{-1}\|V\|\left(B_{2 \theta}^{n+1}(0)\right)<q+1 / 2$ and

$$
q-1 / 2 \leq\left(\omega_{n} \theta^{n}\right)^{-1}\|V\|\left(\mathbb{R} \times B_{\theta}\right)<q+1 / 2 .
$$

For any given $z \in B_{3 / 4}$, we may apply this lemma iteratively starting with $\eta_{(0, z), 1 / 4 \#} V$ in place of $V$, stopping the iteration when we reach a scale at which the first alternative in (a) occurs. Notice that when this happens, the lemma shows that $V\left\llcorner\left(\mathbb{R} \times B_{\sigma}(z)\right)\right.$ for some some $\sigma \in(0,1 / 8)$ is the union of embedded graphs of $q$ smooth functions over $B_{\sigma}(z) \subset \mathbb{R}^{n}$ each with small gradient and solving the minimal surface equation. If the iteration proceeds indefinitely, then spt $\|V\|$ contains precisely of one point above $z$, and $V$ at that point has a unique multiplicity $q$ tangent hyperplane that is almost parallel to $\mathbb{R}^{n} \times\{0\}$, to which $V$ decays upon rescaling. With a little 
extra work, it is not difficult to see that these facts lead to Theorem 6.1 (see [Wic14a], Section 15 for details).

\section{Steps (ii) and (iii): Induction for the Minimum Distance Theorem (and the overall completion of induction)}

The final part of our simultaneous proof of the Sheeting Theorem and the Minimum Distance Theorem is the completion of induction for the Minimum Distance Theorem, i.e. steps (ii) and (iiii) listed in Section 6.3 above. With regard to this, the idea is to complete step (ii) first, using among other things the result of step (i), and then use both step (i) and step (ii) to complete step (iii). In both cases, the argument is by contradiction and uses a version of the blow-up method described in the preceding section, the argument this time being much closer to its original form used by Simon in [Sim93]. More specifically, the goal is to show if there is a sequence of varifolds $V_{j} \in \mathcal{S}$ converging to a cone $\mathbf{C}$ as in the Minimum Distance Theorem (with the appropriate density at the origin, namely, $\Theta(\|\mathbf{C}\|, 0)=q+1 / 2$ in step (ii) and $\Theta(\|\mathbf{C}\|, 0)=q+1$ in step (iii)), then for sufficiently large $j, V_{j}$ must have a classical singularity, in direct contradiction to the definition of $\mathcal{S}$. It is in completing step (ii) and step (iii) that the no-classical-singularities hypothesis is used non-inductively for the first and the only time in the entire proof of Theorem 3.3.

To describe steps (ii) and (iii) in more detail, let $\mathbf{C}$ be as in the Minimum Distance Theorem, i.e. Theorem 6.3. Then $\mathbf{C}=\sum_{j=1}^{m} q_{j}\left|P_{1}\right|$ for some $m(\geq 3)$ distinct half-hyperplanes $P_{1}, P_{2}, \ldots, P_{m}$ meeting along a common boundary $L$ and for some positive integers $q_{1}, q_{2}, \ldots, q_{m}$. If $\eta_{j}$ is the unit vector along $P_{j}$ orthogonal to $L$, it is an easy exercise to show that stationarity of $\mathbf{C}$ in $\mathbb{R}^{n+1}$ is equivalent to the requirement that

$$
\sum_{j=1}^{m} q_{j} \eta_{j}=0,
$$

and to show, using this fact, that

$$
\Theta(\|\mathbf{C}\|, 0) \in\{q+1 / 2, q+1\} \Longrightarrow q_{j} \leq q \text { for each } j=1,2, \ldots, m .
$$

Thus, in view of step (i), we have, whenever the induction hypotheses (H1), (H2) hold and a cone $\mathbf{C}$ and a varifold $V$ are as in Theorem 6.3 with $\Theta(\|\mathbf{C}\|, 0) \in\{q+1 / 2, q+1\}$, then $V$ is regular away from any $\epsilon$-neighborhood of the axis of $\mathbf{C}$ provided $V$ is sufficiently close to $\mathbf{C}$ (depending on $\mathbf{C}$ and $\epsilon)$.

Consider now step (ii), namely, the case when $\Theta(\|\mathbf{C}\|, 0)=q+1 / 2$. In this case, the first task in preparation for the blow up argument is to establish the analogues of Simon's estimates (as in the conclusion of Lemma 7.8 above) for $V \in \mathcal{S}$ satisfying the appropriate set of contradiction hypotheses (analogous to the hypotheses of Lemma 7.8) corresponding to 
step (ii), including the hypothesis that $V$ is close as a varifold to $\mathbf{C}$ in the unit ball $B_{1}^{n+1}(0)$.

Unlike in the case of Lemma 7.8 above, the estimates this time are established following Simon's argument in [Sim93] very closely; the reason why it is possible to do so even though, strictly speaking, the "multiplicity 1 class" hypothesis needed in [Sim93] does not hold in the present situation is because by hypothesis (of the Minimum Distance Theorem with $\Theta(\|\mathbf{C}\|, 0)=q+1 / 2)$, the density ratio of $V$ is not much more than $q+1 / 2$, and that implies, by $(H 1)$ and step (i) (which provides the Sheeting Theorem for multiplicity up to and including $q$ ), complete regularity of $V$ in any small toroidal region around and close to the axis of $\mathbf{C}$ whenever $V$ is close in distance to spt $\|\mathbf{C}\|$ in a slightly larger torus. This fact serves the purpose that Allard's regularity theorem served in the argument of [Sim93]. (See [[Wic14a], Theorem 16.2, Corollary 16.3 and Corollary 16.4].)

Note also that the crucial "no $\delta$ gaps" condition, i.e. the analogue of Lemma 7.8(d), holds in the present setting. For if not, then there is a ball $B_{\delta}=B_{\delta}^{n+1}(Y)$ of fixed size $\delta$ about some point $Y$ on the axis of $\mathbf{C}$ in which all singularities of $V$ have density $<q+1 / 2$, implying, in view of the Almgren-Federer Generalized Stratification Theorem (Theorem 2.1), the induction hypotheses $(H 1),(H 2)$ and Step (i), that $V\left\llcorner B_{\delta}\right.$ is regular up to a singular set of codimension 7 . Since a singular set that small is removable for the stability inequality, the argument described in the first paragraph of Section 6.3 leads to a contradiction whenever $V$ in distance is sufficiently close to $\mathbf{C}$ (depending on $\delta$ ).

Once Simon's estimates are established, a blow up argument analogous to the one described above in the context of Lemma 7.6 can be used to show that when $V \in \mathcal{S}$ is sufficiently close (as a varifold) to $\mathbf{C}$ in $B_{1}^{n+1}(0), V$ must contain a classical singularity somewhere near the origin, contradicting directly the definition of $\mathcal{S}$ and thereby completing step (ii). (See [[Wic14a], Section 16] for details.)

Armed with step (ii), we can repeat the blow up argument, in an identical fashion to how it is used in step (ii) but with the assumption that $\Theta(\|\mathbf{C}\|, 0)=q+1$, to complete step (iii) and consequently the overall induction for both the Sheeting Theorem and the Minimum Distance Theorem.

\section{References}

[All72] W. K. Allard. On the first variation of a varifold. Ann. of Math. 95 (1972), 417-491.

[AA76] W. K. Allard, F. J. Almgren, Jr. The structure of stationary one-dimensional varifolds with positive density. Invent. Math. 34 (1976), 83-97.

[AA81] W. K. Allard, F. J. Almgren, Jr. On the radial behavior of minimal surfaces and the uniqueness of their tangent cones. Ann. of Math. 113 (1981), 215-265.

[Alm65] F. J. Almgren, Jr. Theory of varifolds. Mimeographed notes, Princeton University (1965).

[Alm66] F. J. Almgren, Jr. Some interior regularity theorems for minimal surfaces and an extension of Bernstein's theorem. Ann. of Math. 84 (1966), 277-292. 
[Alm83] F. J. Almgren, Jr. Almgren's big regularity paper: Q-valued functions minimizing Dirichlet's integral and the regularity of area minimizing rectifiable currents up to codimension two. World Scientific.

[CB14-a] C. Bellettini. Uniqueness of tangent cones to positive- $(p, p)$ integral cycles. Duke Math. J. 163 (2014), 705-732.

[CB14-b] C. Bellettini. Semi-calibrated 2-currents are pseudoholomorphic, with applications. Bull. London Math. Soc. 46 (2014), 881-888.

[Ber15] S. Bernstein. Sur un théorème de géometrie et ses applications aux équations dérivées partielles du type elliptique, Comm. de la Soc. Math. de Kharkov (2eme Ser. ) 15 (1915-1917), 38-45.

[BDG69] E. Bombieri, E. De Giorgi, E. Giusti. Minimal cones and the Bernstein problem. Invent. Math. 7 (1969), 243-268.

[SC88] S. Chang. Two dimensional area minimizing integral currents are classical minimal surfaces. J. Amer. Math. Soc. 1 (1988), 699-778.

[CM14] T. H. Colding, W. P. Minicozzi. Generic mean curvature flow I: generic singularities. Ann. of Math. 175 (2012), 755-833.

[CM1] T. H. Colding, W. P. Minicozzi. Uniqueness of blow ups and Lojasiewicz inequalities. arXiv.

[CM2] T. H. Colding, W. P. Minicozzi. The singular set of mean curvature flow with generic singularities. arXiv.

[DG61] E. De Giorgi. Frontiere orientate di misura minima. Sem. Mat. Scuola Norm. Sup. Pisa (1961), 1-56.

[DG65] E. De Giorgi. Una estensione del teorema di Bernstein. Ann. Scuola Norm. Sup. Pisa 3 (1965), 79-85.

[DS] C. De Lellis, E. Spadaro. Regularity of area minimizing currents I-III. arXiv.

[DS11] C. De Lellis, E. Spadaro. Q-valued functions revisited. Memoirs AMS 211 (2011), no. 911

[DT] C. De Lellis, D. Tasnady. The existence of embedded minimal hypersurfaces. Jour. Diff. Geom. 95 (2013), 355-388.

[DP79] M. do Carmo, C. K. Peng. Stable complete minimal surfaces in $\mathbb{R}^{3}$ are planes. Bull. AMS. 1 (1979), 903-906.

[Fed70] H. Federer. The singular sets of area minimizing rectifiable currents with codimension one and area minimizing flat chains modulo two with arbitrary codimension. Bull. Amer. Math. Soc. 76 (1970), 767-771.

[FF60] H. Federer, W. H. Fleming. Normal and integral currents. Ann. of Math. 72 (1960), 458-520.

[FS80] D. Fischer-Colbrie, R. Schoen. The structure of complete stable minimal surfaces in 3-manifolds of non-negative scalar curvature. Comm. Pure and Appl. Math. 33 (1980), 199-211.

[Fle62] W. H. Fleming. On the oriented Plateau problem. Rend. Circ. Mat. Palermo (2) 11 (1962), 69-90.

[GL86] N. Garofalo, F. -H. Lin. Monotonicity properties of variational integrals, $A_{p}$ weights and unique continuation. Indiana Univ. Math. J. 35 (1986), 245-268.

[GL87] N. Garofalo, F. -H. Lin. Unique continuation for elliptic operators: a geometric variational approach. Comm. Pure and Appl. Math. 40 (1987), 347-366.

[HS79] R. Hardt, L. Simon. Boundary regularity and embedded solutions for the oriented Plateau problem. Ann. of Math. 110 (1979), 436-486.

[HS85] R. Hardt, L. Simon. Area minimizing hypersurfaces with isolated singularities. J. Reine Angew. Math. 362 (1985), 102-129.

[Hei52] E. Heinz. Über die Lösungen der Minimalflächengleichung. Nachr. Akad. Wiss. Göttingen Math. Phys. K1 II (1952), 51-56.

[Hug] S. Hughes. Cambridge Thesis (2014).

[HT00] J. E. Hutchinson, Y. Tonegawa. Convergence of phase interfaces in the van der Waals-Cahn-Hilliard theory. Calc. Var. PDE. 10 (2000), 49-84. 
[Ilm93] T. Ilmanen. Convergence of the Allen-Cahn equation to Brakke's motion by mean curvature flow. Jour. Diff. Geom. 38 (1993), 417-461.

[Ilm95] T. Ilmanen. Singularities of mean curvature flow of surfaces. preprint, 1995. http://www.math.ethz.ch/ ${ }^{\sim}$ ilmanen/papers/pub.html

[Ilm96] T. Ilmanen. A strong maximum principle for singular minimal hypersurfaces. Calc. Var. \& PDE. 4 (1996), 443-467.

[KNS78] D. Kinderlehrer, L. Nirenberg, J. Spruck. Regularity in elliptic free boundary problems I. Jour. d'Anal. Math. 34 (1978), 86-119.

[KS89] R. V. Kohn, P. Sternberg. Local minimizers and singular perturbations. Proc. Roy. Soc. Edin. Sect. A 111 (1989), 69-84.

[JK] J. Kolár̆. Non-unique conical and non-conical tangents to rectifiable stationary varifolds in $\mathbb{R}^{4}$. arXiv.

[Krum1] B. Krummel. Existence and regularity of multivalued solutions to elliptic equations and systems. arXiv.

[Krum2] B. Krummel. Constant frequency and the higher regularity of branch sets. arXiv.

[KW1] B. Krummel, N. Wickramasekera. Fine properties of branch point singularities: two-valued harmonic functions. arXiv.

[KW2] B. Krummel, N. Wickramasekera. Fine properties of branch point singularities: two-valued solutions to the minimal surface system. to appear.

[MN12] F. C. Marques, A. Neves. Rigidity of min-max minimal spheres in threemanifolds. Duke Math J. 161 (2012), 2725-2752.

[MN14] F. C. Marques, A. Neves. Min-max theory and the Willmore conjecture. Ann. of Math. 179 (2014), 683-782.

[Men13] U. Menne. Second order rectifiability of integral varifolds of locally bounded first variation. J. Geom. Anal. 23 (2013), 709-763.

[Mod87] L. Modica. The gradient theory of phase transitions and the minimal interface criterion. Arch. Rat. Mech. Anal. 98 (1987), 123-142.

[MM77] L. Modica, S. Mortola. Un esempio di $\Gamma$-convergenza. Un. Mat. Ital. B (5) 14(1) (1977), 285-299.

[Mos77] M. Moschen. Principio di Massimo Forte per le Frontiere di Misura Minima. Ann. Univ. Ferrara - Sez. VII -Sc. Mat. 23 (1977), 165-168.

[Pit77] J. Pitts. Existence and regularity of minimal surfaces on Riemannian manifolds. Princeton University press (1977).

[Pog81] A. V. Pogorelev. On the stability of minimal surfaces. Soviet Math. Dokl. 24 (1981), 274-276.

[Rei60] R. Reifenberg. Solution of the Plateau problem for m-dimensional surfaces of varying topological type. Acta Math. 104 (1960), 1-92.

[Ros10] L. Rosales. The geometric structure of solutions to the two-valued minimal surface equation. Calc. Var. PDE 39 (2010), 59-84.

[Sch04] R. Schätzle. Quadratic tilt-excess decay and strong maximum principle for varifolds. Ann. Sc. Norm. Sup. Pisa Cl. Sci. (5) 3 (2004), 171-231.

[Scho83] R. Schoen. Estimates for stable minimal surfaces in three dimensional manifolds. Ann. Math. Studies 103 Princeton University Press (1983).

[SS81] R. Schoen, L. Simon. Regularity of stable minimal hypersurfaces. Comm. Pure and Appl. Math. 34 (1981), 742-797.

[SSY75] R. Schoen, L. Simon, S. -T. Yau. Curvature estimates for minimal hypersurfaces. Acta Math. 134 (1975), 276-288.

[SU82] R. Schoen, K. Uhlenbeck. A regularity theory for harmonic maps. Jour. Diff. Geom. 17 (1982), 307-335.

[Sha] B. Sharp. Compactness of minimal hypersurfaces with bounded index. arXiv.

[Sim83a] L. Simon. Lectures on Geometric Measure Theory. Proc. Centre Math. Anal. Austral. Nat. Univ., Canberra 3 (1983). 
[Sim83b] L. Simon. Asymptotics for a class of nonlinear evolution equations, with applications to geometric problems. Ann. of Math. 118 (1983), 525-571.

[Sim85] L. Simon. Isolated singularities of extrema of geometric variational problems. Harmonic mappings and minimal immersions, ed. E. Giusti: Lecture Notes in Mathematics, 1161 (1985), 206-277.

[Sim87] L. Simon. A strict maximum principle for area minimising hypersurfaces. Jour. Diff. Geom. 26 (1987), 327-335.

[Sim93] L. Simon. Cylindrical tangent cones and the singular set of minimal submanifolds. Jour. Diff. Geom. 38 (1993), 585-652.

[Sim94] L. Simon. Uniqueness of some cylindrical tangent cones. Comm. Anal. Geom. 2 (1994), 1-33.

[Sim95] L. Simon. Rectifiability of the singular sets of multiplicity 1 minimal surfaces and energy minimizing maps. Surveys in Diff. Geom. Vol II (Cambridge, MA, 1993), International Press, Cambridge, MA. (1995), 1-65.

[Sim96] L. Simon. Theorems on regularity and singularity of energy minimizing maps. Birkhauser (1996).

[SJ68] J. Simons. Minimal varieties in Riemannian manifolds. Ann. of Math. 88 (1968), 62-105.

[SimWic07] L. Simon, N. Wickramasekera. Stable branched minimal immersions with prescribed boundary. Jour. Diff. Geom. 75 (2007), 143-173.

[SimWic] L. Simon, N. Wickramasekera. A frequency function and singular set bounds for branched minimal immersions. to appear, Comm. Pure Appl. Math.

[SW89] B. Solomon, B. White. A strong maximum principle for varifolds that are stationary with respect to even parametric elliptic functionals. Indiana Jour. Math. 38 (1989), 683-691.

[Ste88] P. Sternberg. The effect of a singular perturbation on non convex variational problems. Arch. Rat. Mech. Anal. 101 (1988), 209-260.

[Tay73] J. Taylor. Regularity of the singular sets of two dimensional area minimizing flat chains modulo 3 in $\mathbb{R}^{3}$. Invent. Math. 2 (1973), 119-139.

[Tay76] J. Taylor. The structure of singularities in soap-bubble-like and soap-film-like minimal surfaces. Ann. of Math. 103 (1976), 489-539.

[Ton05] Y. Tonegawa. On stable critical points for a singular perturbation problem. Comm. Anal. Geom. 13 (2005), 439-459.

[TW10] Y. Tonegawa, N. Wickramasekera. Stable phase interfaces in the Van der Waals-Cahn-Hilliard theory. J. Reine Angew. Math. 668 (2012), 191-210.

[TW] Y. Tonegawa, N. Wickramasekera. The blow up method for Brakke flows: networks near triple junctions. arXiv

[Whi83] B. White. Tangent cones to 2-dimensional area-minimizing integral currents are unique. Duke Math. J. 50 (1983), 143-160.

[Whi85] B. White. Regularity of the singular sets in immiscible fluid interfaces and solutions to other Plateau-type problems. Proc. Centre. Math. Anal. Austral. Nat. Univ., Canberra 10 (1985), 244-249.

[Whi97] B. White. Stratification of minimal surfaces, mean curvature flows, and harmonic maps. J. Reine Angew. Math. 448 (1997), 1-35.

[Whi10] B. White. The maximum principle for minimal varieties of arbitrary codimension. Comm. Anal. Geom. 18 (2010), 421-432.

[Wic08] N. Wickramasekera. A regularity and compactness theory for immersed stable minimal hypersurfaces of multiplicity at most 2. Jour. Diff. Geom. 80, no. 1, (2008), 79-174.

[Wic14a] N. Wickramasekera. A general regularity theory for stable codimension 1 integral varifolds. Ann. of Math. 179 (2014), 843-1007. 
[Wic14b] N. Wickramasekera. A sharp strong maximum principle and a sharp unique continuation theorem for stationary codimension 1 integral varifolds. Calc. Var. PDE 51 (2014), 799-812.

[Wic] N. Wickramasekera. Stratification of stable codimension 1 rectifiable currents near points of density $<3$. to appear.

Department of Pure Mathematics and Mathematical Statistics (DPMMS), Centre for Mathematical Sciences, University of Cambridge, United Kingdom

E-mail address: n.wickramasekera@dpmms.cam.ac.uk 